Linköping Studies in Science and Technology

Dissertation No. 2080

\title{
Switching Kinetics and \\ charge Transport in \\ Organic Ferroelectrics
}

\section{Tim Cornelissen}




\title{
Switching Kinetics and Charge Transport in Organic Ferroelectrics
}

\author{
Tim Cornelissen
}

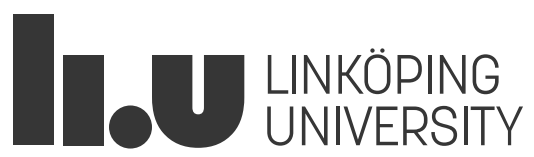

Linköping University

Department of Physics, Chemistry and Biology (IFM)

Complex Materials and Devices

SE-581 83 Linköping, Sweden

Linköping 2020 
Cover image: The stripe domain structure that corresponds to the ground state of the organic ferroelectrics studied in this thesis. This domain structure is characteristic for a hexagonally packed antiferroelectric.

During the course of research underlying this thesis, Tim Cornelissen was enrolled in Agora Materiae, a multidisciplinary doctoral program at Linköping University, Sweden.

This research was funded by the Swedish Government Strategic Research Area in Materials Science on Functional Materials at Linköping University (Faculty Grant SFO Mat LiU No. 2009 00971) and Vetenkapsrådet.

(C) Tim Cornelissen, 2020

ISBN 978-91-7929-828-9

ISSN 0345-7524

URL http://urn.kb.se/resolve?urn=urn:nbn:se:liu:diva-167271

Published articles have been reprinted under the Creative Commons CC-BY(-NC) license.

Printed by LiU-Tryck, Linköping 2020 
Modeling is a lie that makes us realize the truth - Paraphrasing Picasso 



\begin{abstract}
The continued digitalization of our society means that more and more things are getting connected electronically. Since currently used inorganic electronics are not well suited for these new applications because of costs and environmental issues, organic electronics can play an important role here. These essentially plastic materials are cheap to produce and relatively easy to recycle. Unfortunately, their poor performance has so far hindered widespread application beyond displays.
\end{abstract}

One key component of any electronic device is the memory. For organic electronics several technologies are being investigated that could serve as memories. One of these are the ferroelectrics, materials that have a spontaneous electrical polarization that can be reversed with an electric field. This bistable polarization which shows hysteresis makes these materials excellent candidates for use as memories.

This thesis focuses on a specific type of organic ferroelectric, the supramolecular discotics. These materials consist of disk-like molecules that form columns in which all dipolar groups are aligned, giving a macroscopic ferroelectric polarization. Of particular interest are the benzenetricarboxamides (BTA), which are used as a model system for the whole class of discotic ferroelectrics. BTA uses a core-shell architecture which allows for easy modification of the molecular structure and thereby the ferroelectric properties. To gain a deeper understanding of the switching processes in this organic ferroelectric BTA, both microscopic and analytical modeling are used. This is supported by experimental data obtained through electrical characterization.

The microscopic model reduces the material to a collection of dipoles and uses electrostatics to calculate the probability that these dipoles flip. These flipping rates are the input for a kinetic Monte Carlo simulation (kMC), which simulates the behavior of the dipoles over time. With this model we simulated three different switching processes on experimental time and length scales: hysteresis loops, spontaneous depolarization, and switching transients. The results of these simulations showed a good agreement with experiments and we can rationalize the obtained parameter dependencies in the framework of thermally activated nucleation limited switching (TA-NLS).

The microscopic character of the model allows for a unique insight into the nucleation process of the polarization switching. We found that nucleation happens at different locations for field driven polarization switching as compared to spontaneous polarization switching. Field-driven nucleation happens 
at the contacts, whereas spontaneous depolarization starts at defects. This means that retention times in disordered ferroelectrics could be improved by reducing the disorder, without affecting the coercive field. Detailed analysis of the nucleation process also revealed a critical nucleation volume that decreases with applied field, which explains the Merz-like field-dependence of the switching time observed in experiments.

In parallel to these microscopic simulations we developed an analytical framework based on the theory of TA-NLS. This framework is mainly focused on describing the switching transients of disordered ferroelectrics. It can be combined with concepts of the Preisach model, which considers a non-ideal ferroelectric as a collection of ideal hysterons. We were able to relate these hysterons and the distribution in their up- and down-switching fields to the microscopic structure of the material and use the combined models to explain experimentally observed dispersive switching kinetics.

Whereas ferroelectrics on their own could potentially serve as memories, the readout of ferroelectric memories becomes easier if they are combined with semiconductors. We have introduced several molecular materials following the same design principle of a core-shell structure, which uniquely combine ferroelectricity and semiconductivity in one material. The experimental IVcurves of these materials could be described using an asymmetric Marcus hopping model and show their potential as memories.

The combination of modeling and experimental work in this thesis thereby provides an increased understanding of organic ferroelectrics, which is crucial for their application as memories. 


\section{Popular science summary}

The continued digitalization of our society means that more and more things are getting connected electronically. This means that we will find electronics in a large variety of (sometimes unexpected) places. All these new applications put new requirements on the electronics used. Mechanical flexibility, efficiency, cost, and sustainability become more important than just performance. Current electronics, based on hard inorganic metals and semiconductors, cannot always meet these demands.

It is therefore necessary to develop new types of materials for these electronics. One of these new materials are organic electronics. These essentially plastic materials are cheap and easy to produce from abundant resources, relatively easy to recycle, and mechanically flexible. Unfortunately, their relatively poor performance has so far hindered widespread application beyond displays.

One key component of any electronic device is the memory. For organic electronics several technologies are being investigated that could serve as memories. One of these are ferroelectrics, the electrical equivalent of (ferro)magnetism. Both types of memories are based on the material having two stable states between which you can switch, for example by applying a voltage. These states correspond to the logical 0 and 1 that computers use to store information. In the case of magnets it is the magnetic dipole that can either have its north pole up or down, while in ferroelectrics it is the electric dipole which has its positive side pointing up or down.

Ferroelectrics made of organic materials are a relatively new development, and there are only a few materials that are promising for applications. In this thesis we focus on a specific class of materials that consists of small organic molecules. One of the great advantages of these materials is that we can easily tune their properties by slightly modifying the molecules. However, their ferroelectric properties leave room for improvement, which is why we need a deeper understanding of how these materials work.

In this thesis we gain this understanding through two types of modeling, supported by experimental data. The first model describes the material on a microscopic level. It calculates the interactions between all the electric dipoles in the material, and from that it simulates how these dipoles switch between their two states. This allows us to see in detail how the material switches when a voltage is applied and pinpoint any bottlenecks in its performance. From this we found that reducing the disorder in the material morphology is especially crucial. 
The second model is more mathematical and describes the material as a whole. This model allows us to analyze experimental data in terms of microscopic parameters that are specific to a material, which in turn lets us compare different materials and explain their differences in performance. We were also able to directly relate some of these parameters to the disordered morphology of the material.

A problem with ferroelectric memories is checking which of their two states they are in, the so-called readout. When this is done, the memory forgets which state it was in. This means that after every read operation, you have to rewrite the memory. To make a simpler readout possible, ferroelectrics can be combined with semiconductors. Previously this was done by mixing a ferroelectric with a semiconducting material, but we have designed a single material that has both functionalities. Not only does this lead to interesting new physics, it also makes for easier design of ferroelectric memories.

The combination of modeling and experimental work in this thesis thereby provides an increased understanding of organic ferroelectrics, which can be used in their application as memories. 


\section{Populärvetenskaplig Sammanfattning}

Den fortsatta digitaliseringen av vårt samhälle gör att fler och fler saker kopplas samman elektroniskt. Det betyder att vi hittar elektronik på många (ibland oväntade) platser. Alla dessa nya applikationer ställer nya krav på den elektronik som används. Mekanisk flexibilitet, effektivitet, kostnad och hållbarhet blir viktigare än bara prestanda. Nuvarande elektronik, baserad på hårda oorganiska metaller och halvledare, kan inte alltid uppfylla dessa krav.

Det är därför nödvändigt att utveckla nya typer av material för denna elektronik. Ett av dessa nya materialsystem är organisk elektronik. Dessa material, som väsentligen är plast, är billiga och enkla att tillverka från vanligt förekommande resurser, relativt enkla att återvinna, och mekaniskt flexibla. Tyvärr har deras relativ dåliga prestanda hittills hindrat omfattande tillämpning utom i bildskärmar.

En nyckelkomponent i varje elektronisk enhet är minnet. För organisk elektronik undersöks flera tekniker som kan fungera som minnen. En av dessa är ferroelektricitet, den elektriska ekvivalenten till (ferro) magnetism. Dessa typer av minnen är baserade på att materialet har två stabila tillstånd mellan vilka man kan växla, till exempel genom att applicera en spänning. Dessa tillstånd motsvarar de logiska 0 och 1 som datorer använder för att lagra information. När det gäller magneter är det den magnetiska dipolen som antingen kan ha sin nordpol upp eller ner, medan det i ferroelektricitet är den elektriska dipolen som har sin positiva sida pekande upp eller ner.

Ferroelektronik tillverkad av organiska material är ett relativt nytt område, och det finns bara ett fåtal material som är lovande. I denna avhandling fokuserar vi på en specifik klass av material som består av små organiska molekyler. En av de stora fördelarna med dessa material är att vi enkelt kan justera deras egenskaper genom att modifiera molekylerna. Men utvecklingsbehovet för deras ferroelektriska egenskaper är stor, och därför behöver vi en djupare förståelse för hur dessa material fungerar.

I denna avhandling får vi denna förståelse genom två typer av modellering, stödd av experimentella data. Den första modellen beskriver materialet på mikroskopisk nivå. Den beräknar interaktioner mellan alla elektriska dipoler i materialet och simulerar från detta hur dessa dipoler växlar mellan sina två tillstånd. Detta gör att vi i detalj kan se hur materialet växlar när en spänning appliceras och därmed sätta fingret på eventuella flaskhalsar i dess prestanda. Från detta fann vi att det är avgörande att minska oordning i materialets morfologi. 
Den andra modellen är mer matematisk och beskriver materialet som helhet. Denna modell tillåter oss att analysera experimentella data i termer av mikroskopiska parametrar som är specifika för ett material. Detta gör att vi enkelt kan jämföra olika material och förklara deras skillnad i prestanda. Vi kunde också direkt relatera några av dessa parametrar till oordningen i materialets morfologi.

Ett problem med ferroelektriska minnen är att kontrollera vilket av deras två tillstånd de befinner sig i, den så kallade avläsningen. När detta görs på vanligt sätt, glömmer minnet vilket tillstånd det var i. Det innebär att man måste skriva om minnet efter varje avläsning. För att möjliggöra en enklare avläsning kan ferroelektronik kombineras med halvledare. Tidigare gjordes detta genom att blanda ett ferroelektriskt med ett halvledande material, men vi har designat ett enda material som har båda funktionerna. Detta leder inte bara till intressant ny fysik, utan gör det också lättare att skapa ferroelektriska minnen.

Kombinationen av modellering och experimentellt arbete i denna avhandling ger därmed en ökad förståelse av organisk ferroelektricitet, som kan användas i dess tillämpning som minnen. 


\section{Contents}

1 Introduction $\quad 1$

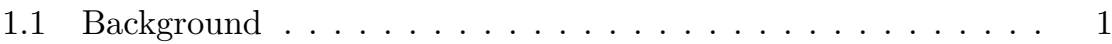

1.1.1 Organic Electronics . . . . . . . . . . . . . . . . . 1

1.1 .2 Ferroelectrics . . . . . . . . . . . . . . 2

1.2 Aim and Outline of Thesis . . . . . . . . . . 3

2 Ferroelectrics $\mathbf{5}$

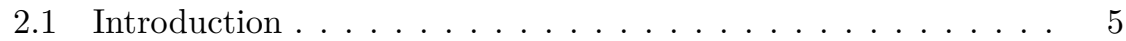

2.2 Classifying Ferroelectrics . . . . . . . . . . . . . 5

2.2.1 Di-, Para-, or Ferroelectric . . . . . . . . . . . 5

2.2.2 Pyro- and Piezoelectricity . . . . . . . . . . . 7

2.2.3 Antiferro- and Ferrielectricity . . . . . . . . . . . . . . 9

2.3 Basic Theory of Ferroelectrics . . . . . . . . . . . . . . . . . . . . . . . . . 9

2.3.1 Polarization..................... 9

2.3 .2 Landau Theory . . . . . . . . . . . . . . . . . . . . . . . . . . . . . . 12

2.4 Ferroelectric Switching in Experiments . . . . . . . . . . . . . . . 12

2.4.1 Ferroelectric Characterization . . . . . . . . . . . 12

2.4 .2 Switching Transients . . . . . . . . . . . . . . 13

2.4 .3 Hysteresis Loop . . . . . . . . . . . . . . . . . . . . 14

2.4.4 Spontaneous Depolarization . . . . . . . . . . . . . 15

2.4 .5 Domains . . . . . . . . . . . . . . 17

2.4.6 Additional Characterization Techniques . . . . . . . . . 18

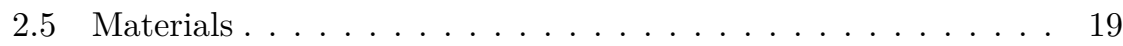

2.5.1 Inorganic Ferroelectrics . . . . . . . . . . . . . 19

2.5.2 Organic Ferroelectrics . . . . . . . . . . . 20

2.5.3 Materials in This Thesis . . . . . . . . . . . . . . 22

2.5.4 Material and Device Fabrication . . . . . . . . 27

3 Modeling Ferroelectrics $\quad 29$

3.1 Introduction . . . . . . . . . . . . . . . . . . . . . . . . . . . 29

3.2 Analytical Models ... . . . . . . . . . . . . . . . . 30

3.2 .1 Nucleation: KAI . . . . . . . . . . . . . . . . 30

3.2 .2 Merz Law . . . . . . . . . . . . . . . . 32 
3.2 .3 TA-NLS . . . . . . . . . . . . . . . . . 33

3.2 .4 Thermally Activated Merz . . . . . . . . . . . . . . 34

3.2 .5 Dispersive Switching . . . . . . . . . . . . . . . 34

3.3 Monte Carlo Models . . . . . . . . . . . . . . . . . . . . . . 41

3.3.1 Introduction . . . . . . . . . . . . . . 41

3.3.2 Monte Carlo Simulations on Ferroelectrics . . . . . . . 43

3.3.3 Kinetic Monte Carlo Simulations on BTA . . . . . . . . . 44

3.4 Molecular Dynamics . . . . . . . . . . . . . . . . . . . . 49

$3.4 .1 \quad \mathrm{MD}$ on Ferroelectrics . . . . . . . . . . . . . 50

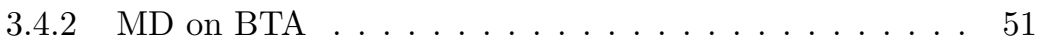

3.5 First Principle Theory . . . . . . . . . . . . . . . . . 52

3.5.1 Density Functional Theory . . . . . . . . . . . . . 52

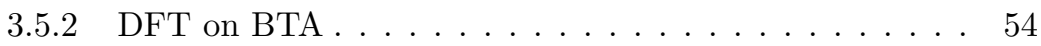

4 Semiconducting Ferroelectrics $\mathbf{5 5}$

4.1 Introduction: Ferroelectric Memories . . . . . . . . . . . . 55

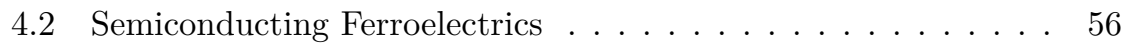

4.2.1 Combining Semiconductors with Ferroelectrics . . . . . . 56

4.2 .2 Inorganic Ferroelectric Semiconductors . . . . . . . . . . 58

4.2 .3 Organic Ferroelectric Semiconductors _ . . . . . . . 59

4.3 Asymmetric Hopping Model . . . . . . . . . . . . . . . . 62

4.3.1 Hopping Charge Transport . . . . . . . . . . . . 63

4.3.2 Asymmetric Charge Transport . . . . . . . . . . . 63

5 Conclusion and Outlook $\quad 67$

$\begin{array}{ll}\text { Acknowledgments } & 71\end{array}$

$\begin{array}{ll}\text { Bibliography } & 75\end{array}$

$\begin{array}{ll}\text { Papers and Contributions } & 93\end{array}$

$\begin{array}{ll}\text { Paper I } & 97\end{array}$

$\begin{array}{lr}\text { Paper II } & 129\end{array}$

$\begin{array}{ll}\text { Paper III } & 151\end{array}$

$\begin{array}{ll}\text { Paper IV } & 181\end{array}$ 


\section{Chapter 1}

\section{Introduction}

\subsection{Background}

\subsubsection{Organic Electronics}

Electronic devices are finding their way into more and more aspects of our daily life. Under the name of the Internet of Things, a lot of things are getting connected electronically. Think of a heart rate sensor integrated into your clothes, or humidity sensors on crops that upload their data to the cloud. All these new applications put new requirements on the electronics being used. Mechanical flexibility, efficiency, cost, and sustainability become more important than outright performance. These new requirements demand a new type of electronics.

The electronic technology that is currently used, consisting of inorganic materials like silicon and copper, has trouble meeting these demands. Fabrication is energy intensive and relatively expensive due to the complicated processing often at high temperatures, as well as scarcity of some of the raw materials. The crystalline structure of the materials makes them rigid and brittle, meaning they would break if bent. The presence of (heavy) metals raises environmental concerns and means that recycling is not straightforward.

This is where organic electronics can make a difference. These electronics are based on organic materials such as polymers or small molecules. These materials are solution processable, allowing easy device fabrication through solution-based coating and printing techniques. This simple device fabrication in combination with easily available base materials allows for mass production that is cheap in both energy and costs. Finally, the lack of toxic materials makes recycling less of an issue. 
Whereas inorganic electronics is a fully matured technology, organic electronics is much newer and therefore still in development. Performance and stability are the main issues hindering broad application, and OLEDs are one of the few examples where organic electronics have really seen broad commercial application. But great progress is being made for e.g. solar cells and transistors, and applications are starting to find their way onto the market $[9]$.

\subsubsection{Ferroelectrics}

One of the key components for any electronic device is the (non-volatile) memory. In inorganic electronics, memories are either electric flash or ferromagnetic tape and hard disks. For organic electronics several potential technologies are being investigated. One of these are devices based on ferroelectric materials, which are the materials of interest in this thesis.

Ferroelectrics are the electric analogue of the more widely known ferromagnets. They consist of electric dipoles that are aligned in a common direction, resulting in an electric polarization as shown in Fig. 1.1. The direction of the dipoles, and thereby the polarization, can be reversed by applying an external electric field. If the applied field is larger than the so-called coercive field, the ferroelectric will switch from one state to the other. This bi-stable switching, signified by the polarization hysteresis loop, is the basis for the memory application of both types of ferroic materials.
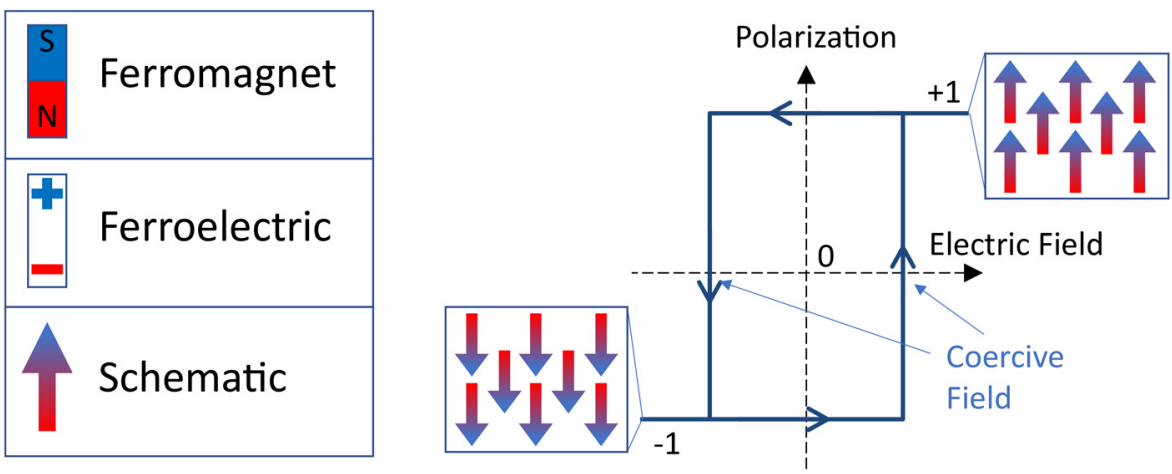

Figure 1.1: Schematic representation of ferroic materials and the hysteresis loop.

Besides memories, ferroelectrics have several other possible applications. One very exciting possibility exploits the symmetry-breaking electric field inside the ferroelectric to facilitate separation of charges generated through the photovoltaic effect. In other words, ferroelectrics might make very efficient solar cells, with output voltages many times larger than the bandgap of the material [10]. Another relatively new area of research is multiferroics, 
materials that exhibit not only ferroelectric but also ferromagnetic and/or ferroelastic properties. The combination and interplay between these properties allow for a variety of new applications [11].

On top of exploiting the ferroelectric properties, ferroelectric materials are also useful for their piezo- and pyroelectric properties. These two properties mean that the polarization of the material also responds to mechanical and thermal stimuli, respectively, which will be discussed in more detail in Section 2.2.2. From an application point of view especially the piezoelectric property is important, due to the direct coupling between voltage and mechanical motion. Piezoelectrics are therefore used in a wide variety of mechanical sensors and actuators.

As holds for most of the field of organic electronics in general, organic ferroelectrics have yet to find their way to commercial success. Generally, their performance is not good enough to compete with already available inorganic materials and technologies. Until recently, the single most promising material for applications was the polymer polyvinylidene fluoride (PVDF) and its derivatives. A memory device based on PVDF was first presented by Asadi et al. [12] and printed memories based on this technique are now commercially available through Xerox [13]. Other organic ferroelectrics have recently started receiving the increased interest of the research community, and while the possibilities are promising, have not been able to reach commercial viability yet.

\subsection{Aim and Outline of Thesis}

Organic ferroelectrics thus still lack in performance compared to their inorganic counterparts. A major reason for this is the lack of understanding of the underlying physical processes that determine the ferroelectric properties of these materials. This thesis aims to address this issue by developing models that are supported by experiments.

The work in this thesis is focused on one specific class of organic ferroelectrics, the supramolecular discotics. These materials consist of disk-like molecules that form columns in which all dipolar groups are aligned. Of particular interest are the BenzeneTricarboxAmides (BTAs), which are used as a model system for the entire class of discotic ferroelectrics. While most work is performed on these BTAs, wherever possible results will be generalized to other organic or disordered ferroelectrics.

The current chapter provides some context and motivation for the research presented in this thesis. Chapter 2 serves as an introduction to the field of ferroelectrics and presents the required concepts, theory, materials, and experimental techniques. Chapter 3 gives an overview of different models used to describe ferroelectric switching, both from literature as well as the microscopic and analytical models developed in this thesis. In Chapter 4 experimental and 
modeling results on semiconducting ferroelectrics are presented. A conclusion and outlook are given in Chapter 5. Finally, the articles that constitute the work in this thesis are included. 


\section{Chapter 2}

\section{Ferroelectrics}

\subsection{Introduction}

This chapter will provide the background and theory relating to ferroelectrics that is necessary to understand the rest of the thesis. We will start in Section 2.2 by first classifying the different types of dielectric materials. This leads us to the ferroelectrics, the basic theory of which will be discussed in Section 2.3. Section 2.4 takes the introduced concepts to the experimental domain and explains the experimental methodology used to study ferroelectrics. Finally, in Section 2.5 we will discuss several different types of ferroelectric materials, as well as the specific materials that are studied in this thesis

\subsection{Classifying Ferroelectrics}

\subsubsection{Di-, Para-, or Ferroelectric}

Classically spoken we consider materials as a collection of positive nuclei surrounded by clouds of negative electrons. While the nuclei are generally considered fixed in space, the electrons are much more mobile. When an electric field is applied, two things can happen. If the material is a conductor, electrons can move freely and will move between atoms along the direction of the field, giving an electric current. If the material is an insulator, the electrons are stuck to their atom, but still have some freedom of movement. They will shift from their equilibrium position, leading to a charge imbalance that results in an electric polarization. In this context the insulator is called a dielectric instead. 
As a first approximation, which is sufficient for low fields, the polarization in a dielectric scales linearly with the electric field $\vec{P}=\epsilon_{0} \chi_{e} \vec{E}$, as shown in Fig. 2.1a. Here $\epsilon_{0}$ is the vacuum permittivity and $\chi_{e}=1-\epsilon_{r}$ the susceptibility of the material. Generally speaking, $\chi_{e}$ is a complex number that depends on frequency, as discussed in Section 2.4.6. In the case of anisotropic materials, the susceptibility becomes a tensor instead of a scalar. The polarization, with unit of $\mathrm{Cm}^{-2}$, can either be regarded as a surface charge on the material or as a dipole density: $\vec{P}=\Sigma \vec{\mu} / V$.

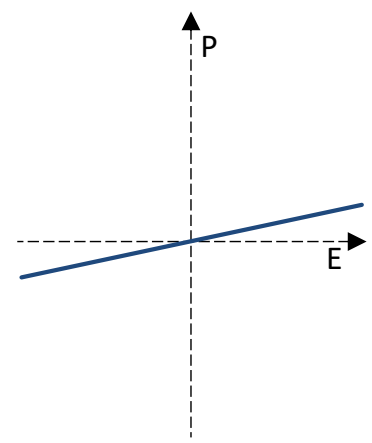

(a) Dielectric

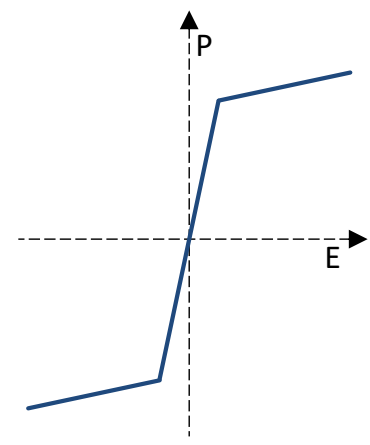

(b) Paraelectric

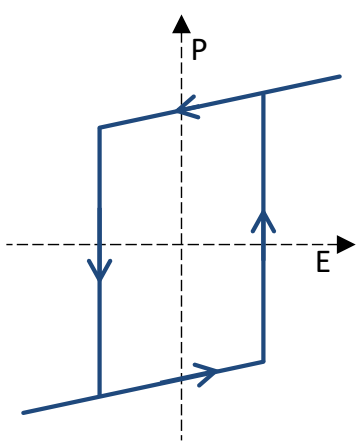

(c) Ferroelectric

Figure 2.1: The polarization as function of applied field.

Besides this electronic polarization, a material might also contain permanent dipoles intrinsically. These dipoles can come from polar molecules such as water or ionic species. Generally, these dipoles will be oriented randomly and any polarization will therefore cancel out. When a field is applied, these dipoles will orient themselves along this field, giving rise to an extra polarization term. A material like this is a paraelectric. The $P(E)$ curve in Fig. 2.1b shows that once all dipoles are oriented, the material is saturated and further increasing the field will only cause more induced electronic polarization. Note that the polarization still vanishes as soon as the applied field is removed.

The distinguishing property of a ferroelectric is that it does have a spontaneous polarization at zero field. This means that the intrinsic dipoles of the material remain aligned even after the field has been removed, in particular along a specific crystal axis. By increasing the field above a certain threshold called the coercive field, the dipoles can be reoriented. This leads to the polarization hysteresis loop in Fig. 2.1c. This loop bears strong resemblance to ferromagnetic hysteresis, and this analogy is where ferroelectrics got their name even though there is no relation to iron (ferrum).

A ferroelectric that is heated up will lose its polarization and become paraelectric. This reversible phase transition, which can be first or second order, is depicted in Fig. 2.2b and will be discussed in more detail in Section 2.3.2. The transition is accompanied by a divergence of the susceptibility at the transi- 
tion temperature. This is again similar to ferromagnets, and the transition temperature is thus also called the Curie temperature $T_{C}$. The susceptibility in the paraelectric phase follows the Curie-Weiss law $\chi_{e}=C /\left(T-T_{C}\right)$.

This section can be summarized in Fig. 2.2a. All insulators can be considered dielectrics, that obtain an induced polarization under an applied electric field. Materials that also have orientable dipoles are paraelectric, gaining an additional polarization component. Some paraelectrics become ferroelectric below their Curie temperature and will have a polarization even at zero applied field.

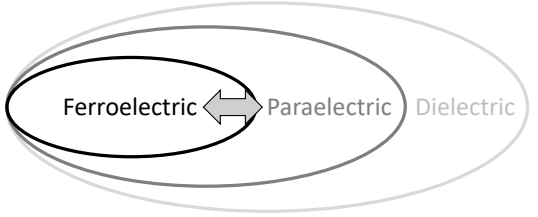

(a)

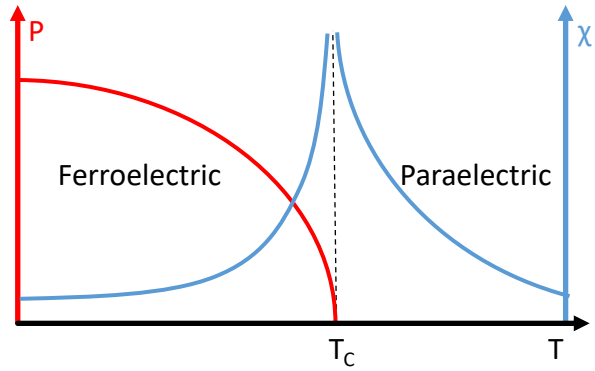

(b)

Figure 2.2: (a) Relation between ferro-, para-, and dielectrics. (b) The polarization and susceptibility as a function of temperature, showing the phase transition from ferro- to paraelectric at the Curie temperature $T_{C}$.

\subsubsection{Pyro- and Piezoelectricity}

A ferroelectric is a thus a material with a spontaneous polarization that is reversible with an applied electric field. If the polarization is (semi)permanent but not reversible we are left with a polar material, which in some cases is also called an electret [14]. The polarization of this material depends on temperature, which is the basis for the pyroelectric effect. As shown in Fig. 2.3b, pyroelectricity is the relation between temperature and polarization, or equivalently the displacement field $\vec{D}=\epsilon_{0} \vec{E}+\vec{P}$. Practically speaking this means that a pyroelectric material generates a voltage when its temperature changes. This makes them very useful as temperature sensors.

The converse effect also occurs and is called the electrocaloric effect. Applying a voltage to a pyroelectric material will lead to a change in entropy and thereby temperature. This is particularly useful for refrigeration applications, as it does not require any moving parts in contrast to classical heat pumps [15]. The strength of both pyroelectric effects is described with the coefficient

$$
p_{i}=\left(\frac{\delta D_{i}}{\delta T}\right)_{E}=\left(\frac{\delta S}{\delta E_{i}}\right)_{T}
$$


where the subscript indicates the direction of the field.

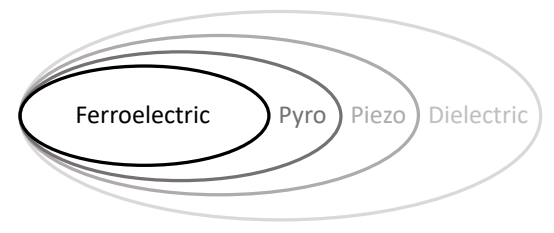

(a)

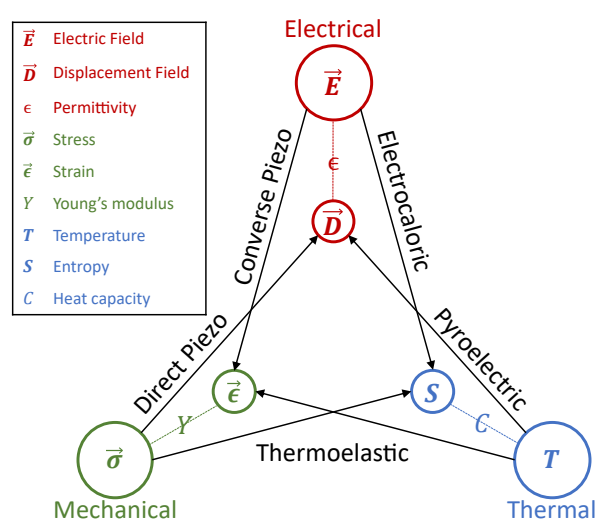

(b)

Figure 2.3: (a) Relation between ferro-, piezo-, pyro-, and dielectric properties. (b) Relation between electrical, mechanical, and thermal properties. Adapted from [16].

Polar materials are also piezoelectric, indicating a relationship between mechanical and electrical properties. The direct piezoelectric effect is the generation of a voltage when a material is deformed, whereas the converse effect means that the material deforms when an electric field is applied. Similarly to pyroelectricity we define the piezoelectric constant as:

$$
d_{i j}=\left(\frac{\delta \epsilon_{j}}{\delta E_{i}}\right)_{\sigma}=\left(\frac{\delta D_{i}}{\delta \sigma_{j}}\right)_{E},
$$

where $i$ indicates the direction of the field and $j$ the direction of the mechanical deformation. Note that $\epsilon$ here is mechanical strain, not the electric permittivity.

Piezoelectricity has proved immensely useful for a large variety of applications. The direct effect allows for pressure sensors, spark generators, and energy harvesters of mechanical motion. The converse effect is widely used in actuators such as loudspeakers and ultrasound generators. Crystal oscillators makes use of both effects and are used for the clocks in most modern electronics.

A piezoelectric material does not necessarily have to be polar, but its crystal structure should lack inversion symmetry. As can be seen in Fig. 2.3a, piezoelectrics are the broadest class of dielectric materials. Any ferroelectric is also pyro- and piezoelectric, but not the other way around. The combination of all these properties makes ferroelectrics incredibly versatile materials for applications. It also provides additional ways to characterize ferroelectrics; for example the pyroelectric current is often used to measure their polarization. 


\subsubsection{Antiferro- and Ferrielectricity}

In a ferroelectric all dipoles are pointing in the same direction. However, when two or more sublattices are present, dipoles might assume an antiparallel configuration, effectively canceling out their polarization. If it is still possible to align all dipoles by applying a sufficiently large field, we call the material antiferroelectric. The characteristic double hysteresis loop of an antiferroelectric is shown in Fig. 2.4b. Note that an antiferroelectric has zero polarization at zero applied field, meaning it will also not be pyro- or piezoelectric there $[17$, 18].

A special case of antiferroelectricity arises when the polarization of the sublattices are not equal. Even when the sublattices are oriented antiparallel, their polarizations will not cancel completely. This leads to the triple hysteresis loop in Fig. 2.4c. A material like this is called ferrielectric, once again analogous to the magnetic case [19].

It should be noted that it is possible for materials to show a mix of these three types of ferroelectricity, and phase transitions between them are possible. With simple electrical characterization it can be difficult to distinguish the different loops, with the added complication that defects and artifacts can produce similar changes in the hysteresis loop [20,21]. Therefore, additional characterization techniques such as X-ray diffraction can be used to investigate antiferrolectricity. Modeling can also be a powerful tool in this regard, since it allows one to directly observe the dipolar orientation, as was done in Paper [3].

Antiferroelectrics have a few interesting possible applications [22]. They can be used for dielectric energy storage, as their pinched hysteresis loops allow for a much larger energy density than in either ferro- or dielectrics [23]. Furthermore, the pyro- and piezoelectric coefficient can be rather large, especially if a phase transition is involved. This is useful for transducer applications that require large strains or high temperature differences [24].

\subsection{Basic Theory of Ferroelectrics}

\subsubsection{Polarization}

Let us have a closer look at the two defining properties of a ferroelectric: the polarization and its bistable switching. In Section 2.2 we stated that polarization can be regarded as a dipole density. While this intuitively works well, it is problematic when applied to bulk materials. This is illustrated in Fig. 2.5 for an infinite 1D chain of dipoles. Depending on your choice of unit cell, the polarization can point in either direction! A practical way to resolve this ambiguity is by only considering changes in polarization instead of its absolute value, which is what is measured experimentally anyhow (see Section 2.4). 


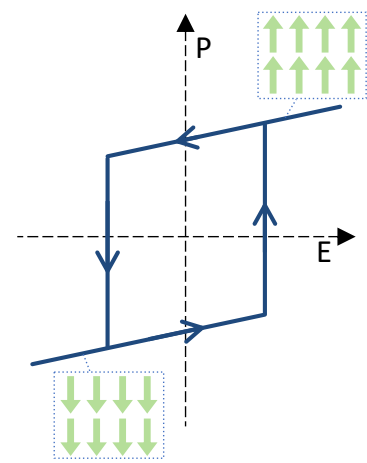

(a) Ferroelectric

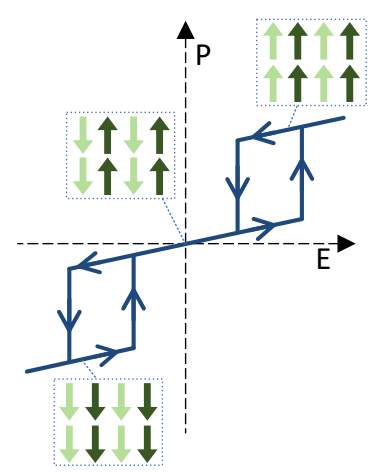

(b) Antiferroelectric

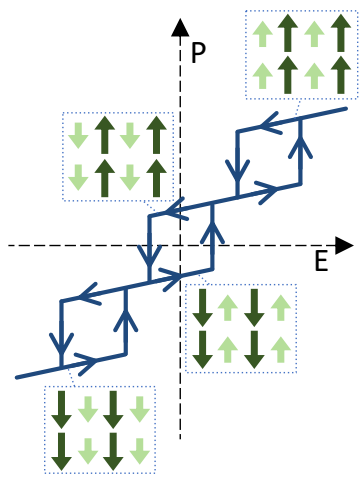

(c) Ferrielectric

Figure 2.4: The polarization as function of applied field and corresponding dipole orientations for different types of ferroic ordering.

A rigorous solution for this simple but fundamental problem was only provided in 1993 by King-Smith, Vanderbilt, and Resta [25, 26]. Their socalled modern theory of polarization uses the concepts of Wannier functions and the Berry phase to unambiguously calculate the polarization of a bulk material. The details are beyond the scope of this thesis, but an accessible description of the theory has been provided by Spaldin [27]. This theory has made it possible to calculate the polarization of materials using first principle methods, as discussed in Section 3.5.

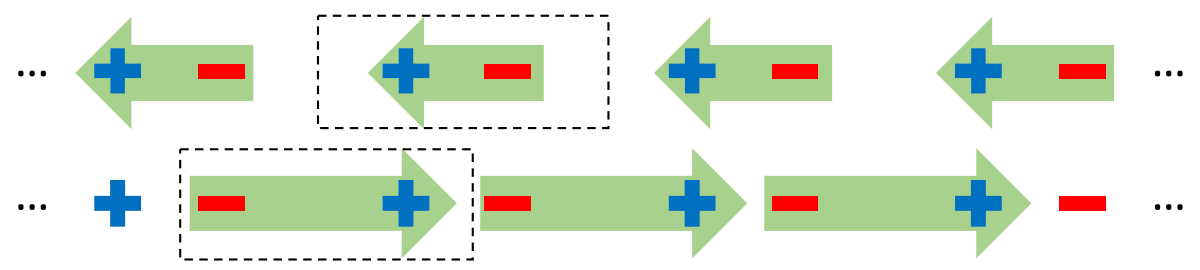

Figure 2.5: Two identical 1D chains of dipoles showing the ambiguity of the direction of polarization depending on choice of unit cell.

\subsubsection{Landau Theory}

That takes care of the polarization itself, but we still need a way to describe its bistability and the switching between the two states. A useful approach here is to use the phenomenological Landau theory. This macroscopic thermodynamic description, formulated by Lev Landau to describe generic phase transitions, was first applied to ferroelectrics by Devonshire [28, 29]. It expresses the free energy density of the system as a power series expansion in 
the order parameter, which for ferroelectrics is the polarization:

$$
G=\frac{1}{2} \alpha P^{2}+\frac{1}{4} \beta P^{4}-E P,
$$

where we truncated the series at the 4 th term, and the coefficients $\alpha$ and $\beta$ determine the nature of the phase transition.

Within Landau theory it is typically assumed that $\alpha=\alpha_{0}\left(T-T_{C}\right)$ is the only temperature dependent parameter, which changes sign at the Curie temperature $T_{C}$. The equilibrium state is obtained by determining the minima of the free energy, i.e. $\delta G / \delta P=0$, which gives

$$
E=\alpha_{0}\left(T-T_{C}\right) P+\beta P^{3} .
$$

We can obtain the susceptibility by differentiating this expression again and setting $P$ to the equilibrium value at zero field $P_{0}$. For $T>T_{C}$ we see that $P_{0}=0$, indicating that this is the paraelectric phase. This finally gives $1 / \chi_{e}=$ $\alpha_{0}\left(T-T_{C}\right)$, i.e. the Curie-Weiss law.

The nature of the phase transition that occurs when going below the Curie temperature is determined by the sign of $\beta$. If $\beta<0$ then the transition is of first order, which means the polarization and susceptibility are discontinuous when passing through $T_{C}$. Here we will focus on the case of second order transitions with $\beta>0$ instead, readers are referred to $[29,30]$ for a treatment of first-order transitions. For $\beta>0$ we find that the polarization has two possible values $P_{0}= \pm \sqrt{\left[\alpha_{0} / \beta\left(T-T_{C}\right)\right]}$, and from that $1 / \chi_{e}=2 \alpha_{0}\left(T_{C}-T\right)$. These expressions correspond to the curves shown in Fig. 2.2b.

The free energy of Eq. (2.3) is graphically shown in Fig. 2.6a. Upon lowering the temperature below $T_{C}$, two equal minima appear in the energy landscape. The minima correspond to the bistable polarization states of the ferroelectric. These two states are separated by an energy barrier $w_{b}=\alpha^{2} / 4 \beta$ which is typically much larger than the thermal energy, which indicates the stability of the states.

When an electric field is applied, the energy landscape is skewed. The two polarization states no longer have equal energy and one state will be favored. However, at low fields the system can remain in the unfavorable state due to the energy barrier. This is the origin of the hysteresis behavior of ferroelectrics, which is graphically shown in Fig. 2.6b. Only when the applied field passes the intrinsic coercive field $E_{c}=\frac{1}{4} \alpha \sqrt{\alpha / \beta}$ will the polarization switch to the other state. This intrinsic coercive field is much higher than that observed experimentally, which will be addressed in detail in Chapter 3 [31-33]. In short, the experimental coercive field can be much lower due to a combination of thermal activation and nucleation processes. 


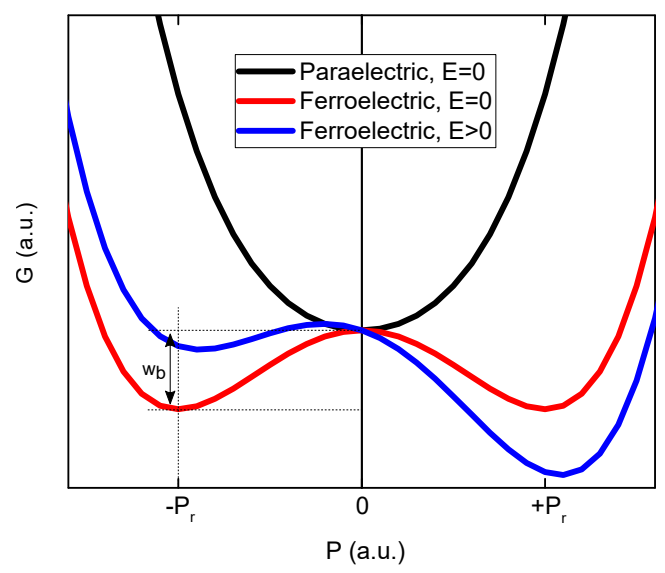

(a)

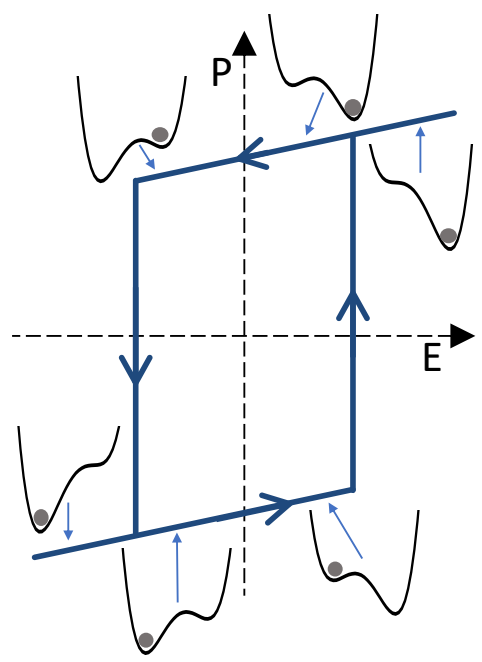

(b)

Figure 2.6: (a) The free energy landscape according to Landau theory in both the para- and ferroelectric phase, with or without applied field. (b) A hysteresis loop showing the polarization switching from the perspective of the free energy landscape.

\subsection{Ferroelectric Switching in Experiments}

\subsubsection{Ferroelectric Characterization}

Now that we have laid a theoretical basis, it is time to face reality and see how the theory applies to experiments. Electrical characterization is the most straightforward way of measuring ferroelectric properties, but care must be taken to interpret data correctly.

A typical setup for performing ferroelectric characterization is shown in Fig. 2.7a. The function generator and amplifier are used to apply an electric field to the ferroelectric capacitor. This causes a change in polarization, which results in a flow of compensation charges to and from the capacitor plates. This current is measured using an oscilloscope. Integrating this current gives the total displaced charge, which when divided by the capacitor area gives the polarization. Note that only changes in polarization are measured this way, as discussed in Section 2.3.1.

This circuit is similar to the so-called Sawyer-Tower circuit used during the initial discovery of ferroelectrics [34]. The use of a function generator and oscilloscope allows one to control the pulse sequence and readout. This provides an advantage over the simple Sawyer-Tower circuit or even commercial ferroelectric testers because specific pulse sequences can be used to compensate 
for any unwanted components in the signal, most notably the displacement and leakage currents.

The Double Wave Method (DWM) shown in Fig. 2.7b is such a pulse sequence [35]. The first pulse is to ensure the sample is fully poled in one direction. The second pulse then switches the polarization, but also measures non-switching current including any leakage and displacement current, as well as any paraelectric polarization changes. The third pulse only measures nonswitching currents, and by subtracting this from the current measured during the second pulse, the ferroelectric switching current is obtained. The same procedure is performed for switching the material the other direction. Without this compensation non-ferroelectric materials such as leaky dielectrics can show hysteresis, as humorously demonstrated by Scott by measuring a banana [36]. More complex pulse sequences can be used to extract even more detailed information about the ferroelectric properties, as demonstrated in Section 3.2.5.3 and Paper [2].

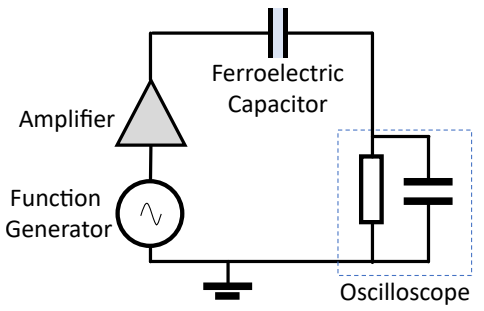

(a)

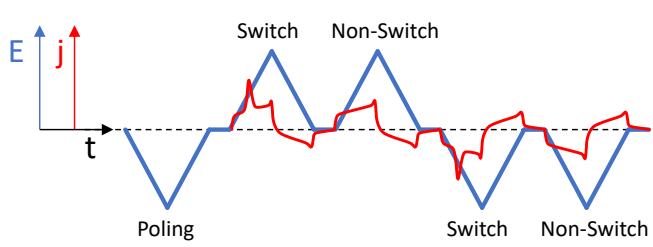

(b)

Figure 2.7: (a) The experimental setup for electrical characterization. (b) The pulse sequence of the DWM.

\subsubsection{Switching Transients}

The simplest experiment that can be done using the setup described above is applying a square voltage pulse to the ferroelectric and measuring its response. This is called a switching transient measurement, and it's mainly used to measure the speed with which the ferroelectric can switch. This speed, represented by the switching time $\tau$, is an important parameter for applications as it will determine the maximum operating frequency of the device.

From theory we would expect that the ferroelectric will switch instantaneously when the applied field is larger than the coercive field. However, reality is significantly more complicated. A typical response to a voltage pulse is shown in Fig. 2.8, from which it is clear that the polarization switches only gradually. This is because it switches through the extrinsic mechanism of nucleation and growth, instead of intrinsic collective switching as predicted from 
basic theory. This process and how it relates to the shape of the switching transient will be discussed in great detail in Chapter 3.

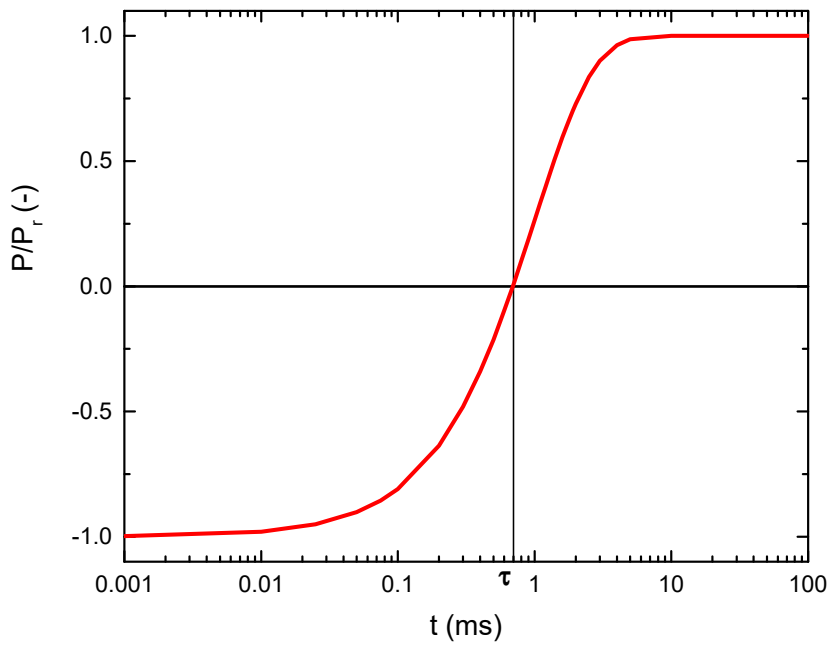

Figure 2.8: Typical polarization response of a ferroelectric after applying a voltage pulse, showing the gradual response and the switching time $\tau$.

It should be noted that the measurement circuit used to measure switching transients is critical for obtaining reliable data. Since switching times can go down to ns $[37,38]$, the electronics used should be able to actuate and measure on this timescale. The circuit used for measuring the switching current should therefore have a low enough RC time. This can be circumvented however by using a series of pulses with increasing length to essentially make snapshots of the switching transient at different times [38]. This way the only limiting factor is the rise time of the applied pulse, which should be significantly lower than the switching time to be measured. This was used in Paper [4] to ensure the electronics were not a limiting factor in switching time measurements.

\subsubsection{Hysteresis Loop}

Hysteresis loops are measured by cycling the electric field back and forth, generally using a triangular waveform as in the pulse sequence shown in Fig. 2.7b. Using the DWM as shown there one can compensate for any displacement or leakage currents that could deform the hysteresis loop. Nevertheless, the hysteresis loop of a real ferroelectric device is not perfectly square, but more rounded as shown in Fig. 2.9.

The rounding of the loop means there is a discrepancy between the remnant polarization (at zero field) $P_{r}$ and the saturation polarization (maximum) $P_{s}$. This indicates that some of the polarization is lost in the time it takes for 


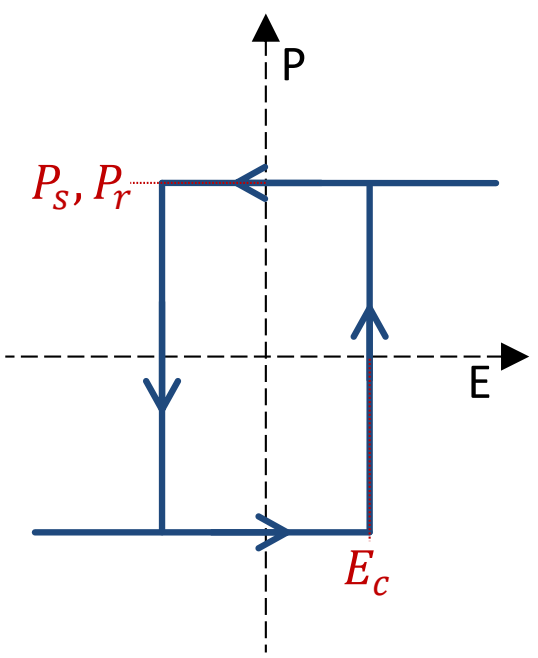

(a)

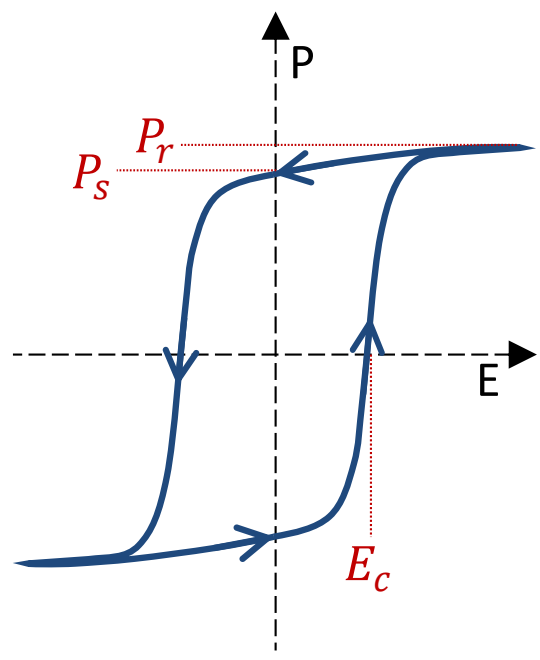

(b)

Figure 2.9: An (a) ideal and (b) real hysteresis loop, showing the coercive field $E_{c}$, saturation polarization $P_{s}$, and remnant polarization $P_{r}$.

the field to cycle back from maximum value to zero. This polarization loss will be discussed in the next section.

It should be noted that the hysteresis loop is highly dependent on experimental conditions such as temperature and measurement frequency. If for example the measurement frequency is higher than the switching speed discussed in the previous section, then the ferroelectric will not have sufficient time to switch between cycles and no polarization will be measured at all. In particular the coercive field is highly dependent on the experimental parameters. A single experimental coercive field does therefore not exist, and it is a meaningless number if experimental details are not included. The dependence of the hysteresis loops on experimental conditions will be discussed in more detail in Section 3.2.3 and Paper [3].

\subsubsection{Spontaneous Depolarization}

In the sections above we have discussed ferroelectric switching as induced by an applied electric field. However, since the switching is a thermally activated process, it can also happen spontaneously. This is generally unwanted, as a long polarization retention is crucial for applications, especially in memories. It is therefore important to understand this depolarization and what causes it.

The main culprit for polarization loss is the depolarization field [39, 40]. This field arises due to the polarization surface charge and is directed opposite to the polarization, as shown in Fig. 2.10a. In a ferroelectric device, 
this surface charge is compensated by the metal electrodes on the surface of the ferroelectric material. However, this compensation is never perfect. The compensation charges can be located a little bit away from the polarization surface charges due to the finite screening length $\lambda$ of the electrodes and 'dead' interface layers.

The imperfect screening of charges gives the depolarization field [41]

$$
E_{\mathrm{dep}}=E_{\mathrm{dep}}^{\mathrm{el}}+E_{\mathrm{dep}}^{\mathrm{int}}=-\frac{P}{\epsilon_{0} d_{f}}\left(\frac{2}{\frac{2}{d_{f}}+\frac{\epsilon_{\mathrm{el}}}{\epsilon_{f} \lambda}}-\frac{d_{\mathrm{int}}}{\epsilon_{\mathrm{int}}}\right),
$$

with $d_{f}$ and $d_{\text {int }}$ the thickness, and $\epsilon_{f}, \epsilon_{\text {int }}, \epsilon_{\mathrm{el}}$ the permittivity of the respective layers. Note that the depolarization field scales inversely with the thickness of the ferroelectric, signifying that its effects are more significant for thin films.

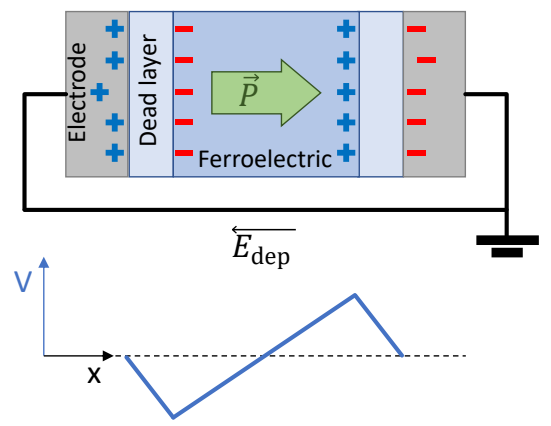

(a)

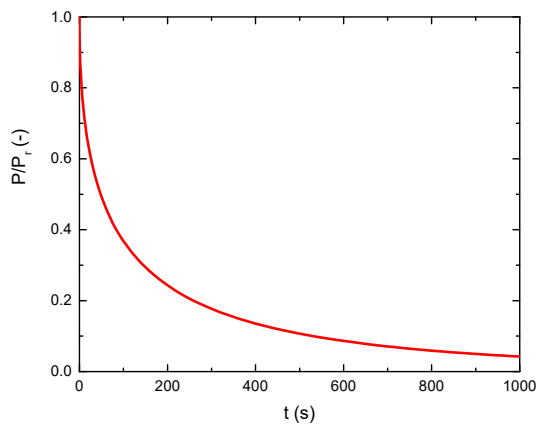

(b)

Figure 2.10: (a) Schematic of the origin of the depolarization field in a shortcircuited ferroelectric capacitor. (b) A typical stretched exponential depolarization curve.

Especially for thin films it can therefore occur that the depolarization field is larger than the coercive field, meaning that the polarization will start to switch. However, since the depolarization field is proportional to the polarization, there is a negative feedback loop. This suggests that this process can continue until an equilibrium is established, where the depolarization field is smaller than the coercive field. However, as discussed before, the switching is a thermally activated process. This means that even a small depolarization field will slowly lead to polarization loss. A variety of models have been used to describe the depolarization, which will be discussed more in Chapter 3. One of the simplest descriptions uses a stretched exponential, which is shown in Fig. 2.10. 


\subsubsection{Domains}

A ferroelectric that got depolarized will generally not lose its polarization completely, at least not on the nanoscale. Instead, domains with their polarization in different directions will form, as shown in Fig. 2.11. This reduces the macroscopic polarization, and thus the depolarization field, while the ferroelectric order is maintained on a smaller scale. We will only briefly discuss domains here, a more extensive treatment can be found in the literature [42, $43]$.

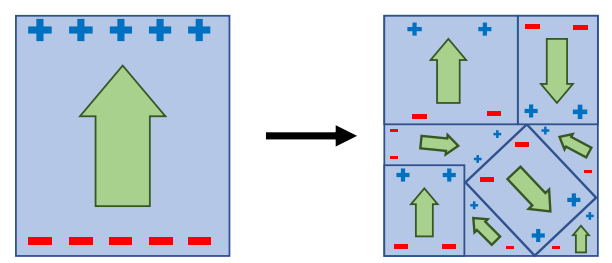

Figure 2.11: A schematic showing the formation of domains, leading to compensation of the polarization surface charges.

Two domains with a different polarization direction are separated by domain walls. Ferroelectric switching happens through the movement of these domain walls, and they have thus been the subject of intense study. Aside from this more fundamental interest, ferroelectric domain walls have several (technologically) interesting properties of their own. For example, they can be conductive even if the bulk material is not [44] and have multiferroic properties [45].

The nanoscale size of domains and domains walls makes direct characterization difficult. A very useful technique for visualizing domains is Piezoresponse Force Microscopy (PFM). This Atomic Force Microscopy (AFM) method uses the piezoelectric effect to map out domains of different polarization on the nanoscale. This can be extremely insightful for visualizing and understanding nucleation and growth of domains [46, 47]. However, care needs to be taken interpreting results from PFM. PFM should not be used to prove ferroelectricity, as surface chemistry plays an important and obfuscating role in generating image contrast $[48,49]$.

Modeling can be of great help here, since it often does allow direct visualization of domains and their kinetics [50]. This holds in particular for molecular dynamics studies as well as Monte Carlo methods, such as the model developed in Paper [3]. These types of models will be discussed extensively in Sections 3.3 and 3.4. 


\subsubsection{Additional Characterization Techniques}

Besides the experimental methods discussed in the previous sections, there are a few additional techniques to characterize ferroelectric properties that are worth discussing briefly.

The first of these is dielectric spectroscopy. This probes the complex permittivity $\epsilon=\epsilon^{\prime}-i \epsilon^{\prime \prime}$ of a material by applying a small AC voltage and measuring both the amplitude and phase of the response. A spectrum can be obtained by sweeping parameters such as temperature, bias voltage, and of course frequency. Depending on the frequency, different types of electrical relaxation processes can be investigated.

Dielectric spectroscopy can be a very useful tool to provide proof of ferroelectricity. Curie-Weiss behavior is a strong indicator of a ferro- to paraelectric phase transition [51]. Another good indicator is the butterfly capacitance vs bias voltage curve in Fig. 2.12. At the coercive field the dipoles start switching and are therefore most responsive to the $\mathrm{AC}$ perturbation, leading to an increase in the permittivity and thus the capacitance of the system.

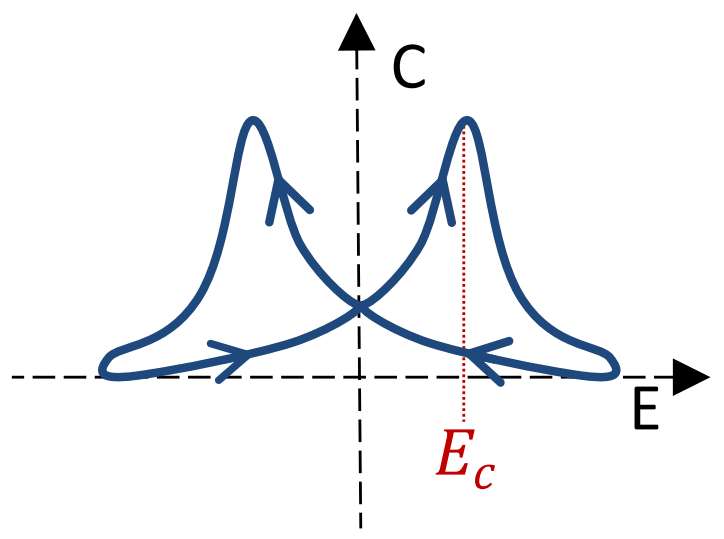

Figure 2.12: A typical capacitance vs bias voltage curve of a ferroelectric.

Another technique that can be used to prove ferroelectricity, or rather the existence of polar order, is Second Harmonic Generation (SHG) [30]. As the name suggests, this technique makes uses of the second order term in the susceptibility relation between polarization and applied field, the linear form of which was introduced in Section 2.2.1. It detects the second harmonic that is generated when light travels through a medium which has a non-linear susceptibility. This can only occur in materials without inversion symmetry, and since the signal is proportional to the square of the polarization, can be used to indicate polar order. The phase of the signal is related to the direction of the polarization, which allows for the determination of polar switching $[52$, $53]$. 
Pyroelectricity can be exploited to measure the absolute value of the polarization through thermal depolarization measurements. A particularly useful extension of this is the Laser Intensity Modulated Method (LIMM). This method uses a pulsed laser to send thermal waves through a polar material and collects the resulting pyroelectric current. By sweeping the frequency of the pulsing and clever demodulation techniques, a map of the polarization as a function of depth can be obtained [54]. This can for example be used to demonstrate the existence of dead layers at the ferroelectric electrode interface [51].

\subsection{Materials}

In the previous sections we discussed the general characteristics of ferroelectrics, without going into any specifics regarding the materials themselves. We will now have a closer look at the different types of materials that exhibit ferroelectricity and their characteristics.

The first ferroelectric material to be discovered, Rochelle salt, is a naturally occurring mineral where hydrogen bonds are responsible for the polar order [55]. Fueled by the technological potential, great advancements have been made since then to make synthetic ferroelectric materials. This has led to an enormous variety of ferroelectric materials being available today. A brief overview will be given here, more comprehensive reviews can be found elsewhere [30, 56-61].

\subsubsection{Inorganic Ferroelectrics}

The field of ferroelectrics has long been dominated by inorganic materials, most notably the perovskite ceramics. These materials have a perovskite structure as shown in Fig. 2.13a, with two cations and an anion (usually oxygen). In the ferroelectric phase the single cation gets displaced from the center of the unit cell. This relative displacement is what causes the dipole moment, and the cation is mobile enough to make switching of the dipole possible.

The most widely used ferroelectric ceramic is lead zirconium titanate $\mathrm{PbZr}_{1-\mathrm{x}} \mathrm{Ti}_{\mathrm{x}} \mathrm{O}_{3}$ (PZT) [56]. It has superior ferroelectric and piezoelectric properties, with a high polarization, moderate coercive field, high piezoelectric coefficient, and good temperature stability. However, the presence of toxic lead is a big issue, which has motivated a search for lead-free materials (as in the fields of perovskite LEDs and solar cells). This has renewed interest in for example barium titanate $\mathrm{BaTiO}_{3}$ (BTO), the first ferroelectric perovskite to be discovered. These materials are often doped with a variety of elements to tune and optimize their properties. While progress is being made, PZT is still the material of choice for most applications [62]. 


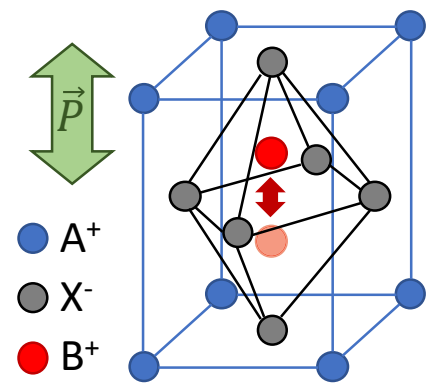

(a) Perovskite

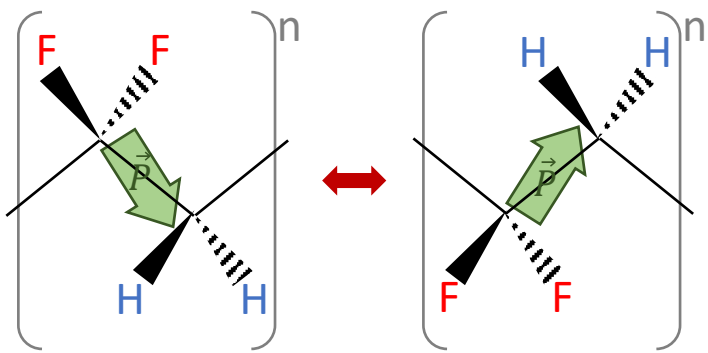

(b) PVDF

Figure 2.13: Structure of a (a) perovskite and a (b) polymer ferroelectric material.

While in the early days mostly single crystal materials were investigated, nowadays polycrystalline materials are used due to easier synthesis. These polycrystalline materials consist of grains that are generally assumed to have a homogeneous polarization, i.e. consist of a single domain. The interaction between the polarized grains then determines the ferroelectric response, and as such control over the microstructure of the material is crucial $[63,64]$. The relation between microstructure, grain interactions, and ferroelectric behavior is the main topic of Paper [2].

Most ferroelectric perovskites fall under the category of displacive ferroelectrics. This means that the phase transition from para- to ferroelectric is associated with some sort of displacement, such as that of the titanium atom in PZT and BTO. The nature of this transition means that in the paraelectric phase the unit cell is no longer polar, as it is inversion symmetric. This is in contrast to ferroelectrics based on the order-disorder mechanism. In this case the unit cell stays polar, but due to microscopic disorder the dipole moments average out and there is no polarization. Ferroelectrics based on reorientable dipolar molecules are an example of this class. In reality the distinction between the two mechanisms is rarely clear-cut, and many ferroelectrics show characteristics of both [65].

\subsubsection{Organic Ferroelectrics}

As discussed in Section 1.1, inorganic materials have several drawbacks related to fabrication, costs, brittleness, toxicity, etc. Organic ferroelectrics could address most if not all these issues. While examples of organic ferroelectrics have been known for decades [66], only recently has the field seen significant expansion powered by the advances in organic chemistry. Several comprehensive reviews are available that discuss the large variety of organic ferroelectrics $[59,60,67]$, here we will briefly discuss some of them. We can classify organic 
ferroelectrics into three groups of materials: polymers, crystals, and liquid crystals.

\subsubsection{PVDF}

The poster child of organic ferroelectrics is without a doubt the polymer PVDF and its various derivatives. The pure polymer consists of repeat units of vinylidene fluoride as shown in Fig. 2.13b. The large electronegativity of the fluorine compared to hydrogen causes a molecular dipole. Its polarization can be flipped through subsequent rotations of the monomers along the flexible polymer backbone. While PVDF had been long since known as a useful plastic, it wasn't until the pioneering work of Furukawa and coworkers that it was shown to be ferroelectric [68, 69].

This was partly due to its high coercive field, but mostly due to the difficulty of getting PVDF into the right crystalline structure [70]. Without further processing it crystallizes in the non-polar $\alpha$-phase, where the dipoles of the subsequent monomers are oriented antiparallel. Only through mechanical drawing or annealing under high pressure can the ferroelectric $\beta$-phase be obtained. This is the reason that a variety of different copolymers have been developed, the most successful one of which is $\mathrm{P}(\mathrm{VDF}-\mathrm{TrFE})$. While these copolymers have a decreased polarization, they crystallize more easily into the ferroelectric $\beta$-phase. Nevertheless, the polymers are semicrystalline with crystallites separated by amorphous matter. This morphology is crucial in understanding their properties such as the negative piezoelectric coefficient [71] and dispersive switching in Paper [2].

The high stability, relatively easy solution processing, good mechanical properties, and decent ferroelectric performance of PVDF polymers have led to it being used for a wide variety of applications, some of which have been successfully commercialized [13]. Their mechanical flexibility makes them uniquely suited for use in flexible electronics [72, 73]. By changing the composition of the copolymer its properties can be tuned for the envisioned application. $\mathrm{P}(\mathrm{VDF}-\mathrm{TrFE})$ has particularly good piezoelectric properties and has been used to make piezoelectric sensors, actuators, and energy harvesters. $\mathrm{P}(\mathrm{VDF}-\mathrm{TrFE}-\mathrm{CFE})$ on the other hand shows very narrow hysteresis and is better suited for electrocaloric or energy storage applications [15]. The ferroelectricity of the polymers has been used for making memory devices, as will be discussed in Section 4.2 [12, 74, 75].

\subsubsection{Crystals}

Organic crystals, crystals from one or two small molecules, can achieve ferroelectricity in two ways $[60,67,76]$. The first is with hydrogen bonds that can be reversed by proton transfer, as is the case in for example coronic acid [77, 78]. The other mechanism is displacive and involves dimerized charge transfer 
complexes [79]. While these organic crystals can have a record-breaking polarization and piezoelectric coefficient, they often only work (far) below room temperature and require careful synthesis of single crystals.

\subsubsection{Liquid Crystals}

The final class of organic ferroelectrics are the liquid crystals. These materials have a unique combination of properties from both liquids and solids that makes them attractive for applications. Their crystallinity allows polar order, whereas their liquidity gives them good processability and mechanical properties. These materials often self-assemble from solution without much need for further processing. Polarizable liquid crystals are already widely used in LCD displays. To achieve ferroelectricity, the material needs to maintain enough order to keep macroscopic alignment of the molecular dipoles. This was first achieved in a chiral smectic phase [80], and since then a large variety of smectic, nematic, and bent-core ferroelectric liquid crystals have been realized [17, 67, 81, 82].

The latest addition to the ferroelectric liquid crystals is that of the columnar discotics, which is the class of materials that is the subject of this thesis $[52,83-85]$. These materials generally follow the core-shell design principle shown in Fig. 2.14a with a $\pi$-conjugated core, a dipolar moiety, and long flexible sidechains. These molecules self-assemble into supramolecular columns as in Fig. 2.14b. The core is either flat or cone-shaped and provides the column stability through $\pi-\pi$ stacking. The dipolar moieties will be the source of the ferroelectric polarization and can further stabilize the columnar structure through dipolar or hydrogen bonding interactions. The sidechains make the molecules soluble and ensure that the system is liquid enough so that the dipoles are free to rotate.

\subsubsection{Materials in This Thesis}

\subsubsection{BTA}

This brings us to the materials that are the focus of this thesis, the supramolecular discotics. The archetypal compound of this class of materials are the trialkylbenzene-1,3,5-tricarboxamides, or BTAs in short. This simple molecule shown in Fig. 2.15 follows the core-shell principle outlined in the previous section and Fig. 2.14. The core consists of benzene to which three dipolar amide groups are attached. Attached to these amides are flexible alkyl tails, that can be substituted by a large variety of alkylic compounds, which will be discussed in more detail below.

As introduced for the discotics in general, BTA will self-assemble in solution into supramolecular columns. What is special in BTA is the triple helix of hydrogen bonded dipoles that forms as shown in Fig. 2.15b. The out-of-plane angle of the dipolar amide groups is around $30-40^{\circ}$, which together with the 


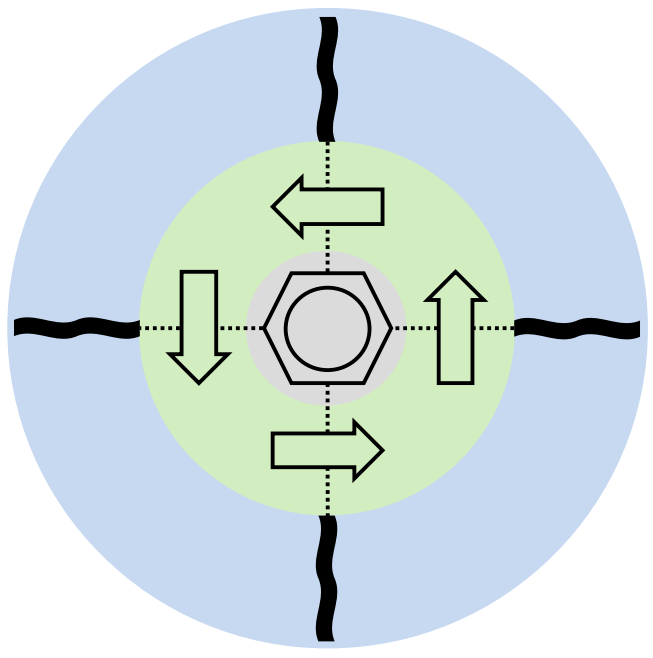

(a)

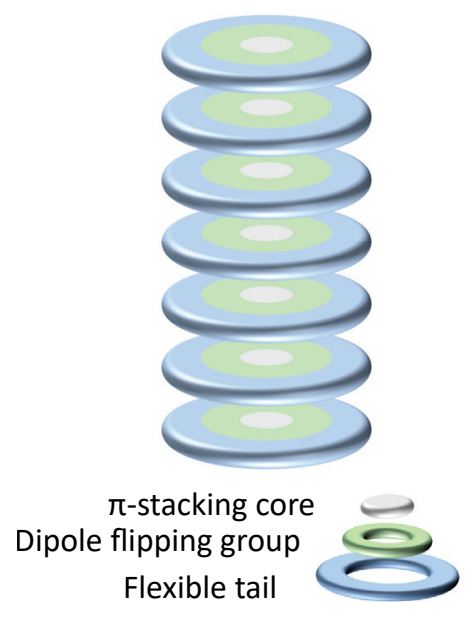

(b)

Figure 2.14: Schematic structure of a columnar discotic liquid crystal. The (a) core-shell structure of a discotic molecule that stacks into (b) supramolecular columns.

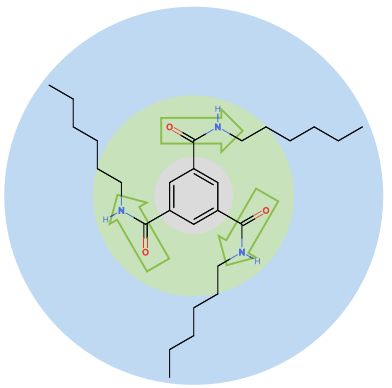

(a)

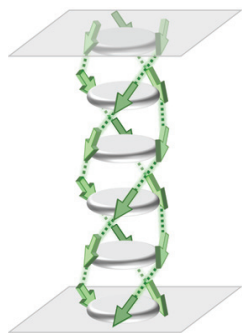

(b)

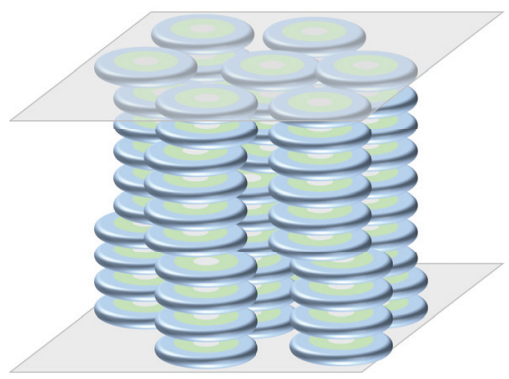

(c)

Figure 2.15: The (a) primary, (b) secondary, and (c) tertiary structure of BTA, in this case with a C6 alkyl tail.

length of the hydrogen bond and the $\pi-\pi$ packing distance leads to a helical pitch of 6 molecules $[86,87]$. This means that each molecule is rotated by $60^{\circ}$ with respect to the adjoining molecules, which nicely aligns with the C6 symmetry of the benzene core.

BTAs are of great interest to the organic chemistry community due to their simple structure but unique and complex self-assembly properties [88]. Their self-assembly is highly cooperative, meaning that each subsequent monomer that attaches to the growing oligomer stabilizes the oligomer more than the previous monomer did [89]. This high cooperativity is for a large part due 
to the macrodipole that forms through the alignment of the amide dipoles. The self-assembly is even more interesting due to the chirality effects that can occur. Using chiral sidechains one can control the chirality of the triple helix, and mixing molecules with different chirality can lead to interesting chiral amplification effects [90].

Going from solution to the more solid state, the BTA columns will organize in a hexagonal packing as shown in Fig. 2.15c. This is largely driven by the steric repulsion between the tails, and so it is these tails that to a large degree govern the spacing and arrangement of the columns. At low temperatures the material will be crystalline and the side tails will be 'frozen'. At intermediate temperatures it will be in the liquid crystalline state, where the tails and amide groups are fairly mobile, but the columns remain ordered. At high temperatures the material melts into an isotropic liquid where there is no long range order left.

It should be noted that in the (liquid) crystalline state there is a good amount of disorder. The columns are divided into subcolumns by defects, as depicted in Fig. 2.15c. At these defects the hydrogen-bonded helix is broken and a translational as well as rotational offset may be present. The length of the subcolumns depends on the exact side-tails and is a measure for how disordered the material is. Disorder might also exist on a larger scale with more amorphous regions of disorganized molecules, in a way not unlike the amorphous regions in semicrystalline polymers like PVDF. Both types of disorder will turn out to be crucial to understand the ferroelectric properties, and this disorder is therefore a recurrent theme in our body of work $[2,3$, $5-7]$.

\subsubsection{Ferroelectricity of BTA}

As mentioned, a macrodipole arises within a BTA column when all dipoles are pointing along the same direction. The amide groups are free to rotate due to the mobility provided by the flexible tails. This provides the basis for the ferroelectricity in BTA; by applying an electric field the dipoles, and thereby the polarization, can be switched between pointing up or down [91, 92]. This results in the typical hysteresis loop shown in Fig. 2.16. The polarization found here is close to the value calculated from the geometric dipole density.

Besides these close to ideal hysteresis loops, there is further proof that BTA is a proper ferroelectric. The most convincing of those is that it displays Curie-Weiss behavior, with a divergence of the dielectric susceptibility at the Curie temperature [51]. Furthermore, it shows typical butterfly CV curves as in Fig. 2.12 [5], is pyroelectric [51], as well as piezoelectric [7]. It is interesting to note that the piezoelectric coefficient $d_{33}$ of BTA is negative, a rather unique property it shares PVDF, which is a result of the disordered microstructure of the material [7]. 


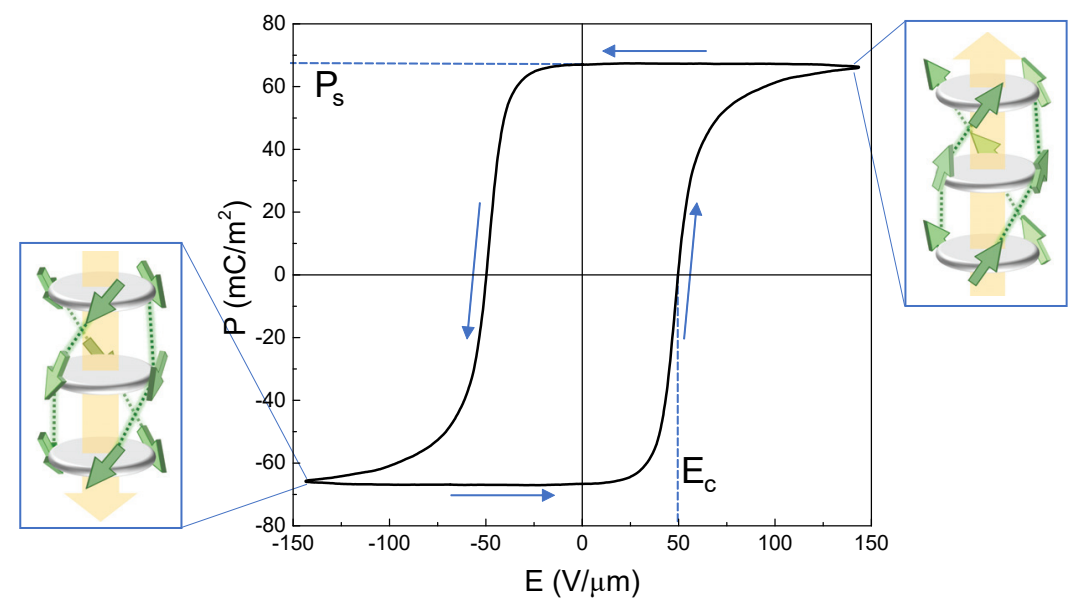

Figure 2.16: A typical hysteresis loop of BTA, showing the dipoles flipping between down and up.

\subsubsection{Tuning the Properties of BTA}

The properties of BTA can easily be tuned by changing the alkyl tails of the molecules. The simplest change is made by changing the length of the linear alkyl tails. This affects the crystallinity of the system, and change the phase transition temperatures, which determine the operation temperature range of a device. Furthermore, it changes the packing parameters of the liquid crystal, in particular the intercolumnar distance. This in turn greatly influences the ferroelectric properties, as demonstrated by the hysteresis loops in Fig. 2.17 $[5]$.

The decreased intercolumnar distance for shorter tails leads to a larger dipole density and thereby a larger polarization. In fact, the increase in polarization is even larger than would be expected geometrically, which was attributed to an increase in intercolumnar interaction and decrease in disorder. This decrease in disorder, combined with the lower mobility of the shorter tails, leads to an increase of the barrier for polarization switching $w_{b}$. Consequently, the coercive field as well as the retention time are highest for the shortest tail length of C6.

Another way of changing the tails is by introducing branches in the alkyl chain. This leads to a enhancement of the self-assembly properties and thereby a decrease in disorder in the liquid crystal. This results in ferroelectric devices with retention times that are essentially infinite at lower (but above room) temperatures [6]. However, the already high coercive field of BTA increases even further to practically inconvenient values. This can be addressed by mixing branched and linear tails, which generally leads to intermediate values for the ferroelectric parameters, providing a tool for continuous tuning. In 


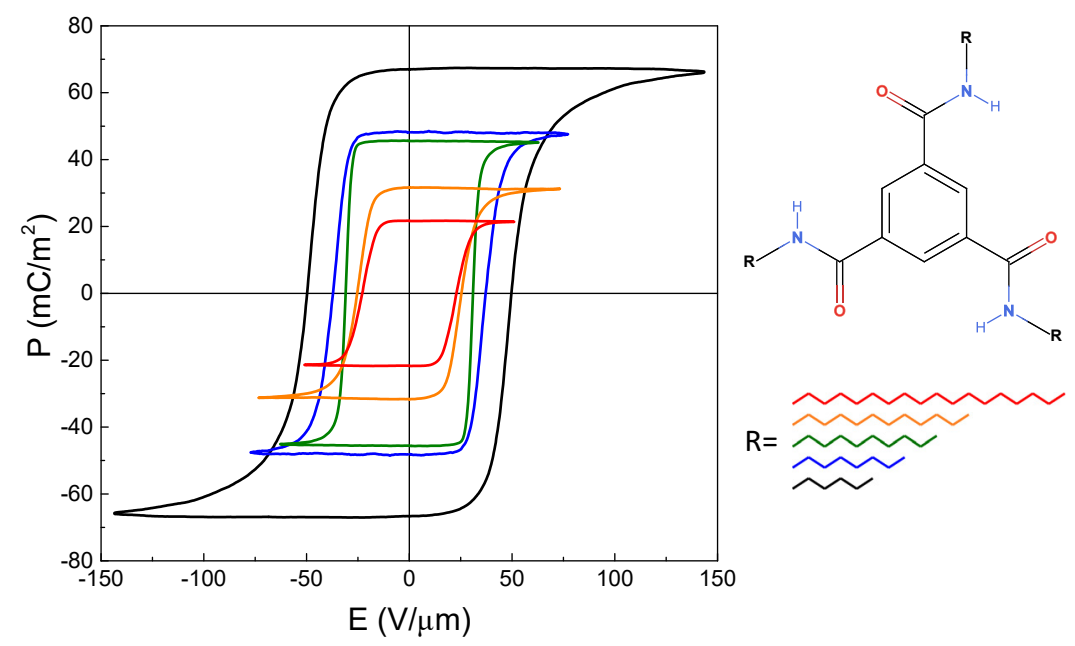

Figure 2.17: Changing the hysteresis loop of BTA by changing the alkyl tail length. [5]

some cases mixing even has a synergistic effect, where the resulting retention time is larger than that of the constituent homologues [6].

\subsubsection{Semiconducting Cores}

Moving inward in the core-shell structure, the next possible modification is that of the dipolar moiety. However, this goes beyond the scope of this thesis and for a treatment of different types and number of dipolar moieties we refer to the literature $[59,67,85]$. That leaves the core as the final possibility for modifications.

The $\pi$-conjugation of the BTA core provides extra stability to the supramolecular columns through $\pi-\pi$ stacking. Without this $\pi$-conjugation similar molecules, such as CyclohexaneTrisAmide (CTA), can still selfassemble into columns but they are less stable and do not exhibit ferroelectricity $[59,93]$. The $\pi$-conjugation extends throughout the entire column, leading to partial electron delocalization, making BTA technically a semiconductor. However, the small size of benzene means that the bandgap is around $5 \mathrm{eV}$, with an absorption in the deep UV [86, 94]. This is too large to be of any practical relevance, and we therefore consider BTA an electrical insulator. It should be noted that the ionic conductivity in discotic liquid crystals can be significant [95-97], which needs to be considered to correctly interpret electrical characterization data.

By increasing the size of the core, we can increase the $\pi$-conjugation and thereby reduce the bandgap. This has been extensively done for nonferroelectric discotic liquid crystals, leading to the discovery of several high 
mobility organic semiconductors [95, 96, 98]. In these systems the charge transport is (quasi-)1D through the core of the column, with the charge carriers hopping between molecules.

The semiconducting ferroelectrics from our work that will be discussed in this thesis are the Carbonyl-Bridged Triarylamine-cored trisamide ( $\alpha$-CBT), SubPhthaloCyanine-BF-trisamide (SubPc-Amide), and BenzeneTrisThiophene-Trisamide (BTTTA). As can be seen in Fig. 2.18, these molecules still follow the same core-shell design principle. Their ferroelectric and semiconducting properties, as well as the interesting interplay between these two, will be discussed in Section 4.2.3 and Paper [1].

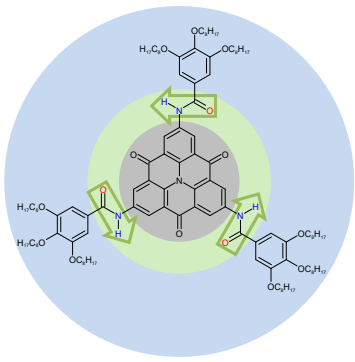

(a) $\alpha-\mathrm{CBT}$

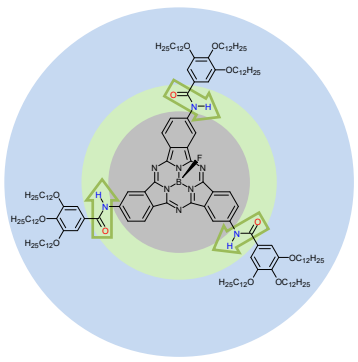

(b) subPc-Amide

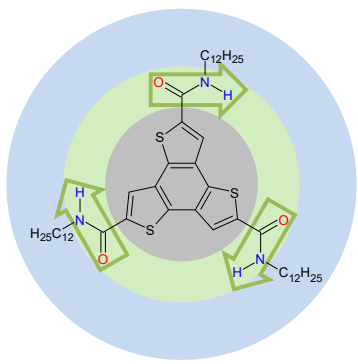

(c) BTTTA

Figure 2.18: The three main semiconducting ferroelectrics studied in this thesis.

\subsubsection{Material and Device Fabrication}

A large variety of discotic molecules similar to the ones studied in this thesis have been studied previously by organic chemists because of their interesting self-assembly properties. In most cases standard synthesizing procedures could therefore be followed, where the core and the amides plus tails are synthesized separately and then attached in the final step before purification. The details on this synthesis can be found in the respective publications and references therein $[8,53,88,99-101]$.

Standard characterization techniques are used to verify the structure and morphology of the synthesized materials. Molecular structure is confirmed using Nuclear Magnetic Resonance (NMR). The presence of hydrogen-bonding can be determined using InfraRed (IR) spectroscopy. Differential Scanning Calorimetry (DSC) measures the heat flow into a material while scanning the temperature, which allows the determination of phase transition temperatures and enthalpies. Together with (temperature dependent) X-ray diffraction this allows one to construct the phase diagram of the material. The semiconducting compounds can be further analyzed using cyclic voltammetry and UV-Vis spectroscopy to determine energy levels and the band gap. 
One of the great advantages of organic electronics is that they are solution processable. This makes for rather straightforward device fabrication. Powder materials are dissolved in an appropriate solvent and can then be deposited. As mentioned, ferroelectric materials are characterized in a capacitor device structure as in Fig. 2.10a. There are several ways to obtain such a geometry. The simplest of those is to use so-called Liquid Crystal Cells (LCC), which consist of two glass plates with electrodes that are separated by a spacer. The material can be entered into the cell through capillary filling. A second method is to evaporate patterned contacts onto a glass substrate, spincoat or dropcast the material, and then evaporate the top electrode. In these two configurations the device has an out-of-plane structure. Alternatively, an in-plane device can be made by using interdigitated electrodes on which the material is again spincoated or dropcasted.

The material will not immediately organize in the ordered fashion shown in Fig. 2.15c. Instead, the material will form disorganized in-plane bundles as can be seen with AFM $[5,51]$. An alignment procedure is therefore necessary, which consists of applying an electric field at elevated temperatures. The dipoles in the molecules and columnar aggregates will then align themselves along the field. In case of transparent electrodes, this alignment can be directly visualized using Polarized Optical Microscopy (POM). After this, the devices are ready to be characterized electrically using the methods described in Section 2.4. 


\section{Chapter 3}

\section{Modeling Ferroelectrics}

\subsection{Introduction}

This chapter presents an overview of the different models that exist to describe ferroelectrics, which includes several of our own contributions. These models are important in understanding the physics governing ferroelectrics, which in turn is crucial for applications. This overview is by no means exhaustive, and will mainly focus on the models that are relevant for describing the switching kinetics in disordered ferroelectrics such as the organic materials studied in this thesis. Several reviews are available in the literature where similar overviews are presented from different perspectives [30, 50, 102].

We classify the models based on the level of detail of their description. The highest level models are the most accurate, but also the most computationally expensive, as shown in Fig. 3.1. We will start by discussing the lowest level models, the analytical models in Section 3.2. These give a macroscopic description in terms of analytic equations. They are derived for example from phenomenological descriptions or energy arguments. Their great advantage is that they are intuitive and that the equations can directly be fitted to experimental data. However, the physical insight these models provide is limited.

One step more detailed are the (kinetic) Monte Carlo methods discussed in Section 3.3. These types of models reduce a material to a collection of microscopic units, generally the dipoles in case of ferroelectrics. The dynamics of the system are calculated through thermodynamic considerations of the interactions between these units. Input parameters can be obtained from higher level theories or calibrated to experimental results. These models are simple enough to be able to provide results on experimental length and time scales, but they do strongly simplify all interactions involved. 


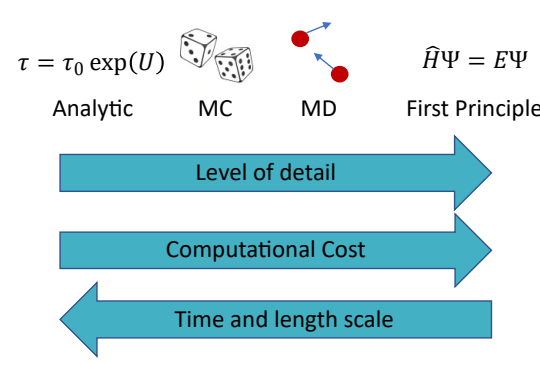

(a)

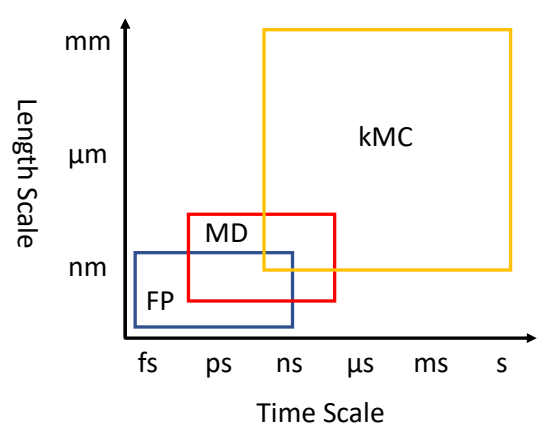

(b)

Figure 3.1: A comparison between the different types of models discussed in this chapter: analytical, Monte Carlo (MC), Molecular Dynamics (MD), and First Principles (FP).

A step further we have Molecular Dynamics (MD) calculations, presented in Section 3.4. These describe the physical movements of atoms or groups of atoms by numerically solving Newton's equation of motion. The forces between the atoms can be approximated to various level of accuracy depending on the system being simulated. MD simulations can describe small systems consisting of several hundreds of atoms on time scales just below those accessible experimentally.

The highest level of models are those based on first principles in Section 3.5. These methods try to directly solve the quantum mechanical Schrödinger equation. Depending on the implementation, few approximations and input parameters are necessary. However, choosing the right methods and approximations is difficult. Furthermore, the computational costs of these methods are so large that generally only information about the ground state can be obtained for systems of only a few unit cells.

\subsection{Analytical Models}

The models and theories presented in this section are aimed at describing the macroscopic switching kinetics of ferroelectrics. We focus specifically on the switching transients (see Section 2.4.2), as this is the simplest form of switching kinetics that can be studied. Hysteresis loops, with their cyclic loading, are slightly more complicated, but can in principle be described by extending the models for the switching transients.

\subsubsection{Nucleation: KAI}

In a real ferroelectric material, polarization switching occurs through a process of nucleation and growth, rather than a homogeneous collective reversal. This 
significantly lowers the theoretical energy barrier for polarization switching as introduced in Chapter 2. A typical representation of this process is schematically shown in Fig. 3.2.

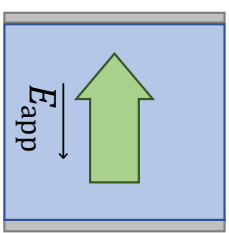

(a)

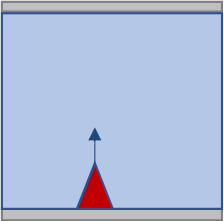

(b)

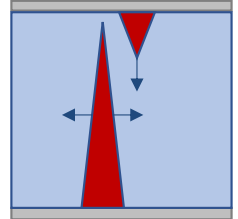

(c)

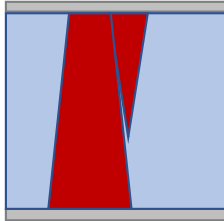

(d)

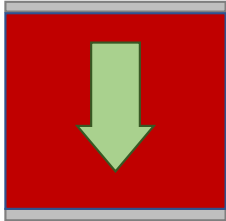

(e)

Figure 3.2: The process of nucleation and growth.

The first step in the switching process is the nucleation of reversed domains. This nucleation is usually inhomogeneous and occurs at preferential sites such as defects or electrodes. These nuclei will then expand preferentially in the direction of the applied field. Finally, the domains will expand laterally until they coalesce and the material is fully switched.

This process of nucleation and growth is classically described by a model developed by the group of Ishibashi based on crystallization theories from Kolmogorov and Avrami [103-105]. This so-called KAI model assumes random nucleation throughout the material and unrestricted growth of the switched domains with a constant velocity. This velocity is assumed to be the limiting factor in the switching process rather than the nucleation rate. This gives for the polarization as function of time:

$$
\Delta P(t)=2 P_{s}\left(1-\exp \left(-(t / \tau)^{d}\right)\right)
$$

where $\tau$ is the characteristic switching time that is inversely proportional to the domain wall speed. $d$ is a dimensionality factor, for which we need to distinguish two cases. If nucleation happens only instantaneously at the beginning of the switching, $d$ equals the dimensionality of the domain growth. If nucleation continues throughout the process, the dimensionality of the growth is $d-1$. However, distinguishing between the two cases is not straightforward. As a result, $d$ is often left as a fitting parameter resulting in non-integer values with unclear physical meaning.

The group of Ishibashi further extended their model to also describe hysteresis loops, and found a relation between the coercive field and the frequency of the applied field as $E_{c} \propto f^{d / a}[106,107]$. Here $a$ is an empirical factor depending on the waveform used. A correction on the KAI model was made by Shur et al., who included an impingement time to account for the finite size of a real ferroelectric [108].

The KAI model has been shown to be valid for certain systems and in particular single crystals [106, 108, 109]. However, it fails to explain the 
switching of polycrystalline thin films, especially the slowdown observed at lower fields and longer time scales $[38,40,110,111]$. Similarly, the switching in organic ferroelectric BTA was found too dispersive to be accurately described by KAI $[2,51]$. There are two major reasons for the failure of the KAI model, the first being that switching in polycrystalline materials cannot be described by a single switching time $\tau$. The second reason is that KAI assumes that domain wall motion is the limiting factor in the switching process, whereas this is often the nucleation instead. Another drawback of the KAI model is that it does not say anything about the influence of for example the applied field or the temperature on the switching process. Models that address these shortcomings will be addressed below.

\subsubsection{Merz Law}

The switching time $\tau$ is dependent on the applied electric field: at higher fields the ferroelectric will switch faster. This dependence of the switching time on the electric field was first described by Walter Merz in 1954 for $\mathrm{BaTiO}_{3}$. This semi-empirical description has become known as the Merz law:

$$
\tau\left(E_{\text {app }}\right)=\tau_{\infty} \exp \left(E_{\text {act }} / E_{\text {app }}\right),
$$

where $\tau_{\infty}$ is the switching time in the limit of infinite field, and $E_{\text {act }}$ the so-called activation field [112].

The Merz law has proved to be remarkably accurate for a wide range of materials, such as single crystals [112], bulk ceramics [113], thin films $[38,111]$, organic-inorganic composites [114], and purely organic ferroelectrics $[115,116]$. However, several deviations have been observed, particularly at high fields [37, 111, 117-119]. Most notably, the supramolecular organic ferroelectrics that are the focus of this thesis could not be described by the Merz law $[5,51]$. Instead, an adapted version as introduced by Fatuzzo was used, which includes an extra $E^{-n}$ term in the prefactor $[120,121]$.

A more fundamental issue with the Merz law is its unclear physical origin. It is generally considered to be associated to the nucleation process, which in turn governs the domain wall speed and the switching time. Equivalently, it has been described as creep behavior of the ferroelectric domain walls in a pinning potential $[42,122-126]$.

In particular the meaning of the activation field $E_{\text {act }}$ has been the subject of much interest [42, 122, 123, 127-130]. Miller and Weinreich derived an expression for the activation field by calculating the energy of a critical triangular nucleus [127], but their calculation overestimated the activation fields by an order of magnitude. Molecular dynamics simulations by the group of Rappe have demonstrated that the shape of the nucleus is generally more complex, which reduces the calculated activation field closer to more experimental values [122, 123]. More recently, Zhao et al. have connected the 
activation field to the depolarization field [130]. Nevertheless, it remains unclear how universally valid these different insights are and how they translate to for example supramolecular organic systems.

\subsubsection{TA-NLS}

A more fundamental model for ferroelectric switching was developed by Vopsaroiu et al. [131, 132]. Their theory of Thermally Activated Nucleation Limited Switching (TA-NLS) assumes that the switching rate of a material is determined by the switching of its pre-existing nucleation sites. The theory starts from the Landau free energy landscape as it was introduced in Section 2.3.2 and Fig. 2.6. It considers the switching between the two states as activation over the energy barrier $w_{B}$ by the combination of temperature, applied field, and depolarization field. When an electric field is applied, the energy of the states is changed by $\pm P_{s} E_{\text {app }} V^{*}$, where $V^{*}$ is the critical volume of the nucleation site. The energy difference between the two states is the starting point of a non-equilibrium statistical model with a master equation that describes the occupation probabilities of the states.

Within this framework, the authors obtain a description of the polarization switching transient that is equivalent to the KAI Eq. (3.1) with $d=1$. They find for the switching time as a function of both temperature and applied field:

$$
\tau=\frac{1}{\nu_{0}} \exp \left[\frac{\left(w_{b}-P_{s} E_{\mathrm{app}}\right) V^{*}}{k_{B} T}\right],
$$

where $k_{B}$ is the Boltzmann constant. The attempt frequency $\nu_{0}$ describes how often the energy barrier is attempted to be overcome, which is generally associated with a typical phonon frequency of the material. Note that by setting $E_{\text {app }}=0$, an expression for the retention time as a function of temperature is obtained. Interestingly, while this expression correctly describes the Arrhenius like temperature dependence of the switching time, its linear field dependence is at odds with the inverse dependence of the Merz law. While Eq. (3.3) might be able to give optically satisfying fits to experimental switching times over a narrow range of fields, it fails to correctly describe the Merz-like behavior that is found in most materials.

From the switching time, also an expression for the coercive field can be derived:

$$
E_{c}=\frac{w_{b}}{P_{s}}-\frac{k_{B} T}{V^{*} P_{s}} \log \left(\nu_{0} t_{\exp } / \log (2)\right),
$$

where $t_{\exp } \propto 1 / f$ is the duration of the experiment, i.e. the inverse voltage sweeping frequency. The first term $w_{b} / P_{s}=E_{c \text {,in }}$ represents the intrinsic coercive field, while the second term is the extrinsic reduction due to thermal activation of the nucleation process. Note that the requirement that the coercive field stays positive puts an upper time limit on the validity of this equation. 


\subsubsection{Thermally Activated Merz}

In the previous two subsections we have seen two different approaches to describe the dependence of the switching time on both applied field and temperature. Unfortunately, the more insightful theory of TA-NLS $(\log (\tau) \propto-E)$ does not agree with the experimentally validated Merz $\operatorname{law}(\log (\tau) \propto 1 / E)$. In Paper [4] we address this issue and reconcile the two theories.

The key insight for this reconciliation is provided by examining the nucleation process in BTA using a microscopic Monte Carlo model, which will be discussed in detail in Section 3.3. Simulations with this model show that the size of the critical nucleus, which corresponds to the highest point in energy during the nucleation, changes with the applied field. In particular, we find that the nucleation volume in Eq. (3.3) is inversely proportional to the applied field: $V^{*}\left(E_{\text {app }}\right)=V_{0} E_{V} / E_{\text {app }}$, where $V_{0}$ is the effective volume of a single dipole, and $E_{V}$ a characteristic field. Inserting this expression into Eq. (3.3), we get

$$
\tau=\frac{1}{\nu_{0}} \exp \left(\frac{-E_{V} V_{0} P_{s}}{k_{B} T}\right) \exp \left(\frac{w_{b} V_{0}}{k_{B} T} \frac{E_{V}}{E_{\text {app }}}\right) .
$$

In other words, the Merz law of Eq. (3.2) is obtained, with more transparent expressions for its two parameters:

$$
\begin{aligned}
\tau_{\infty} & =\frac{1}{\nu_{0}} \exp \left(\frac{-E_{V} V_{0} P_{s}}{k_{B} T}\right), \\
E_{a} & =\frac{E_{V} w_{b} V_{0}}{k_{B} T} .
\end{aligned}
$$

We find that Eq. (3.5) provides a good description of both experimental as well as simulation results on BTA, as shown in Fig. 3.3. Values for the energy barrier $w_{b}$ are consistent with those found when fitting the coercive field to Eq. (3.4). The inverse temperature dependence of the activation field has been observed in many systems, both experimentally [42, 111, 115, 116, $124]$ as well as in simulations $[122,123]$. Our description also provides an expression for the prefactor $\tau_{\infty}$, which is often overlooked in literature or assumed to be constant with both field and temperature. In the literature that does investigate it, a variety of different dependencies is found [111, 115, $116]$.

\subsubsection{Dispersive Switching}

The models introduced in the previous sections assume that the material can be described by a single switching time $\tau$. While this works for single crystals, most ferroelectrics (both organic and inorganic) are poly- or semicrystalline. This means that the materials are inherently inhomogeneous, with regions (e.g. crystal grains) that can behave differently. It therefore 


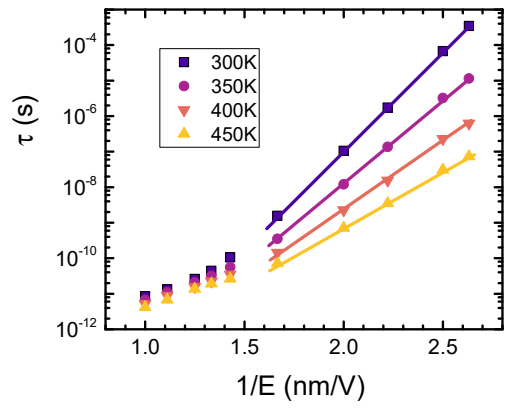

(a) Simulation

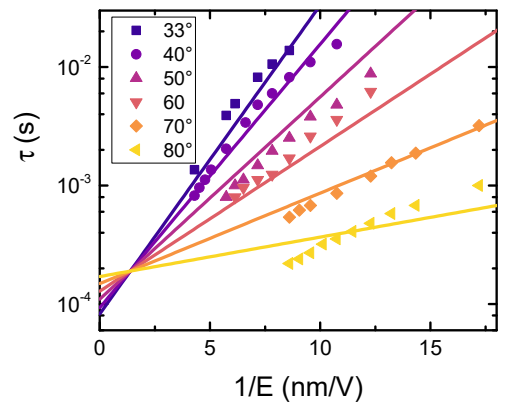

(b) Experiment

Figure 3.3: The switching time as a function of applied field in both (a) kinetic Monte Carlo simulations and (b) experiments on BTA. Both datasets are fitted to the thermally activated Merz Eq. (3.5).

makes sense to consider the materials as a collection of perfect regions (e.g. grains, crystallites) that each have their own properties and environment. Instead of single, well-defined, material parameters, we then need to consider distributions of these parameters. This is the basis for the models in this section that describe the dispersive switching of polycrystalline ferroelectrics.

\subsubsection{Nucleation Limited Switching}

The first model that introduced a distribution of switching times was proposed by Tagantsev et al. [38]. Their Nucleation Limited Switching (NLS) model considers a ferroelectric as an ensemble of non-interacting elementary regions. These regions have a characteristic size and switching time, and switch instantaneously once that time is reached, so that domain wall motion within the regions is neglected. Using some reasonable approximations, this model leads to the following polarization transient:

$$
\Delta P(t)=2 P_{s} \int_{-\infty}^{+\infty}\left[1-\exp \left(-(t / \tau)^{d}\right)\right] N(\tau) d \tau
$$

which reminds of the KAI Eq. (3.1), but then with $N(\tau)$ as the distribution of switching times.

In the original work of Tagantsev, the authors used a phenomenological mesa-like distribution to fit Eq. (3.8) to the experimental switching transients measured on PZT thin films. Instead, Jo et al. used a log-Lorentzian distribution which they ascribed to the distribution of local fields inside the film [111]. Their approach works well for a large range of fields and temperatures, and they find that the centers of the distributions follow the Merz law. For BTA, we found that a Gaussian distribution works best to describe the switching 
transients [51], which will be discussed in more detail in Section 3.2.5.3. An overview of these distributions and their corresponding switching transients is presented in Fig. 3.4.

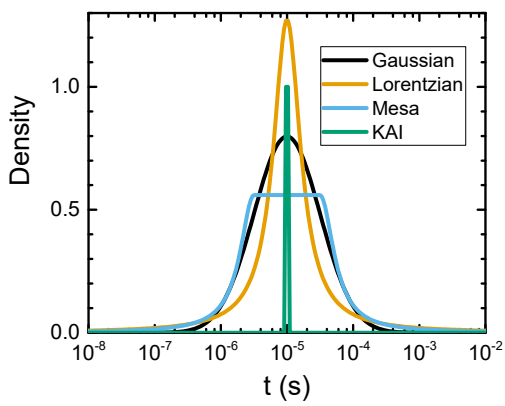

(a)

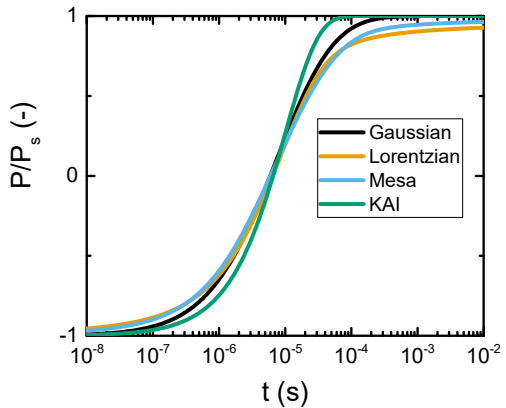

(b)

Figure 3.4: (a) The switching time distributions and (b) resulting switching transients of various implementations of the NLS model.

\subsubsection{Inhomogeneous Field Mechanism}

While the NLS theory works well to describe dispersive switching, the origin of the switching time distribution remains unclear. This was addressed by the Inhomogeneous Field Mechanism (IFM) introduced by Zhukov et al. [113, 133]. As the name suggests, it derives the distribution of switching times from the inhomogeneous and time dependent distribution of the local electric fields inside the film. This distribution of local fields is a direct consequence of the microstructure of the material.

The great advantage of the IFM is that it presents a methodology to extract these distributions (be it local fields or switching times) from experimental data. By scaling the logarithmic derivative of the switching transients obtained at different fields, one can obtain a universal master curve, the shape of which reflects the inhomogeneous field distribution. Using this methodology, the authors found a close to Gaussian switching time distribution in PZT, resulting in the switching transient

$$
\Delta P(t)=P_{s} \operatorname{erfc}\left[\frac{\frac{E_{a}}{E_{\mathrm{app}} \log \left(t / \tau_{\infty}\right)}-1}{\sigma \sqrt{2}}\right],
$$

where $\sigma$ is the width of the distribution. In further work, the same group refined their model which emphasized the connection between microstructure and switching time distribution, and applied the model to a variety of different polycrystalline ferroelectrics $[63,64,134]$. 


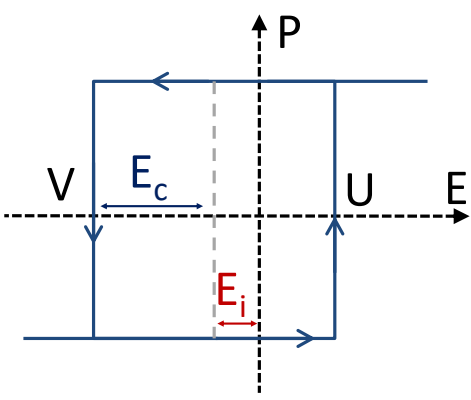

(a)

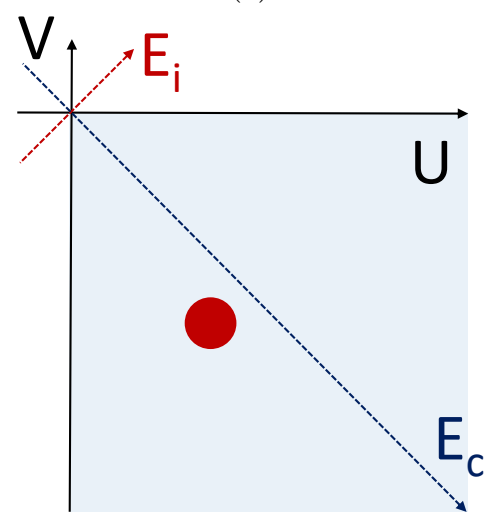

(c)

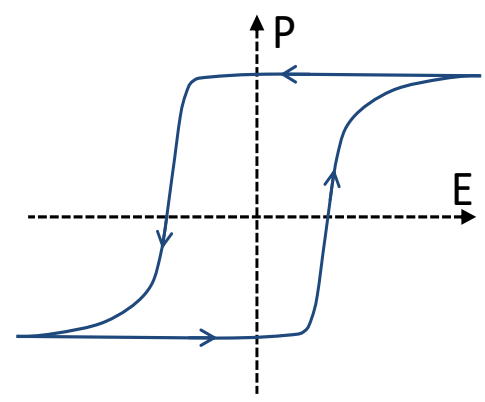

(b)

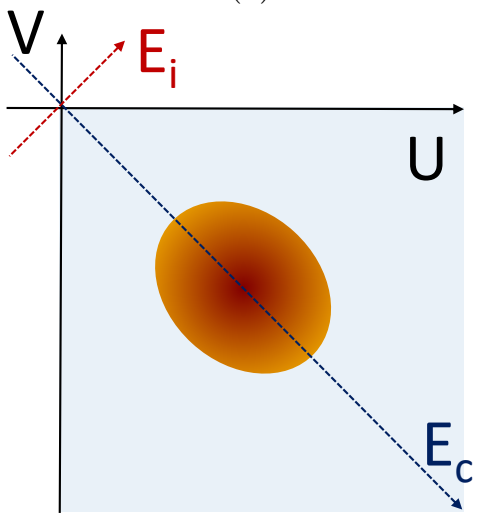

(d)

Figure 3.5: (a) An ideal hysteron and (c) its location on the Preisach plane. (b) A real hysteresis loop consists of a collection of hysterons and is represented as (d) a distribution on the Preisach plane.

\subsubsection{Preisach Model}

\section{Introduction}

The idea of modeling a ferroic material as an ensemble of ideal units is not new. It was first introduced by Ferenc Preisach in 1935 for describing hysteresis loops in ferromagnets [135]. He posed that a real hysteresis loop could be described as the sum of a collection of ideal so-called 'hysterons'. These elementary hysterons have a perfectly square hysteresis loop as in Fig. 3.5a, with an up-switching field $U$ and a down-switching field $V$. Equivalently, they can be described by a coercive field $E_{c}=(U-V) / \sqrt{2}$ and internal offset field $E_{i}=(U+V) / \sqrt{2}$. A material consists of a collection of hysterons with a distribution in these parameters, leading to the slanted hysteresis loop in Fig. 3.5b.

This distribution is often visualized on the Preisach plane spanned by the parameters $U$ and $V$. Note that we generally are only interested in the quadrant of the plane where $U>0$ and $V<0$, as the other quadrants imply 
nonphysical or highly imprinted behavior. A single hysteron is represented by a point as in Fig. 3.5c, whereas a real material is described by the $2 \mathrm{D}$ Preisach Distribution (PD) shown in Fig. 3.5d. Sweeping the applied field as during a hysteresis loop measurement is equivalent to sweeping along one of the axes of the Preisach plane. The switched polarization is then proportional to the integral of the PD: $P(E>0) \propto \int_{-\infty}^{+\infty} \int_{-\infty}^{E} \mathrm{PD}(U, V) d U d V$.

Due to the mathematical nature of the model, it is not just restricted to ferromagnets and can easily be applied to other hysteretic systems [136]. Its application on ferroelectrics was first suggested by Turik in the 60s [137], but it wasn't until a few decades later that experimentalists started using it to describe ferroelectric hysteresis loops. Most of the initial research has been on inorganics, and focused on macroscopic measurements of the hysteron distribution, as well as on using the model to predict hysteresis loop behavior [138-143]. The use of the Preisach model for organic ferroelectrics has mostly been limited to $\mathrm{P}(\mathrm{VDF}-\mathrm{TrFE})$ and its copolymers [144-147].

The Preisach models bears resemblance to the dispersive switching models of the previous sections, with the difference that it is aimed at described the polarization as a function of applied field (hysteresis loops) rather than time (switching transients). And like those models, the physical origin of the distributions remains disputed. Using advanced PFM techniques, the group of Kalinin has performed local measurements of the PD, providing some insight into the microscopic mechanisms underlying it [148-150].

\section{The Physical Basis of the Preisach Model}

In Paper [2], we apply the Preisach model to both BTA and P(VDF-TrFE), and find a physical basis for the model in disordered ferroelectrics. Using specifically designed pulse sequences, we can write and read specific areas of the PD. This allows us to make a detailed measurement of the $2 \mathrm{D} \mathrm{PD}$, rather than relying on $1 \mathrm{D}$ projections as is usually done. We find that both $\mathrm{PDs}$ can be described well by a double Gaussian, which is often the assumed form in literature. The shape of both PDs is markedly different however; whereas the $\mathrm{PD}$ of $\mathrm{P}(\mathrm{VDF}-\mathrm{TrFE})$ in Fig. 3.6b is more cigar-shaped with a low spread in $E_{i}$, the distribution in BTA is mostly round as in Fig. 3.6a.

We relate this difference to the microstructure of the materials as shown in Figs. 3.6c and 3.6d. As discussed in Section 2.5.3, BTA consists of tightly packed columns, which through defects are separated into subcolumns of variable length. $\mathrm{P}(\mathrm{VDF}-\mathrm{TrFE})$ is a polycrystalline material consisting of crystallites surrounded by an amorphous matrix. We pose that these subcolumns and crystallites correspond to the hysterons in the Preisach model. In the case of BTA, the subcolumns electrostatically interact with each other, resulting in a spread of the local internal field $E_{i}$, similar to the IFM model. For $\mathrm{P}(\mathrm{VDF}-\mathrm{TrFE})$, the crystallites are electrically shielded from each other by the amorphous phase, and therefore $E_{i}$ is close to 0 . The spread in $E_{c}$ is then 


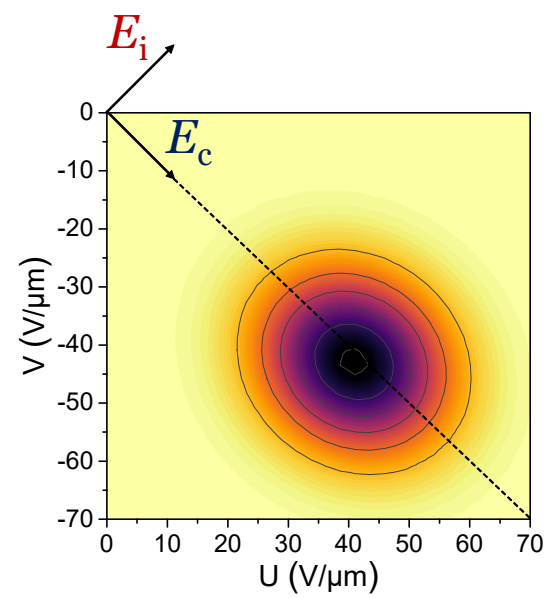

(a)

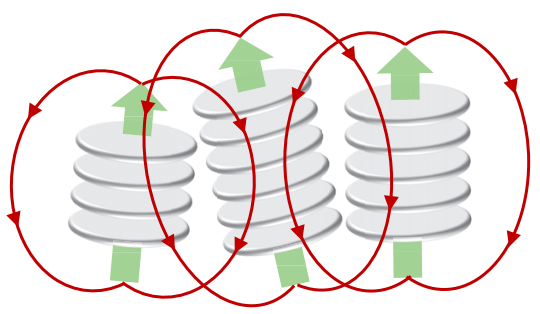

(c)

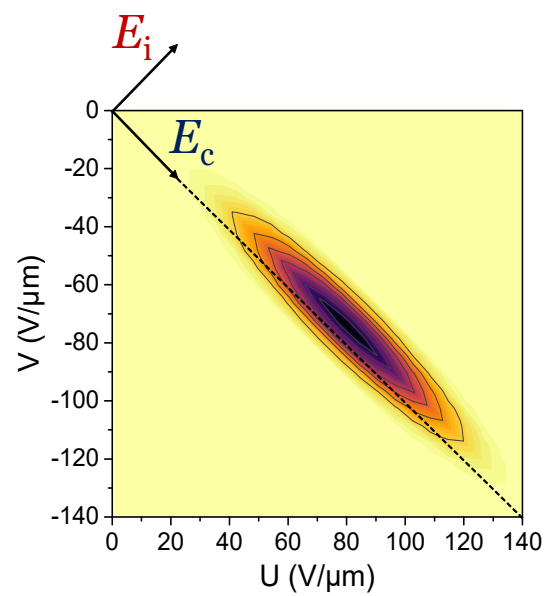

(b)

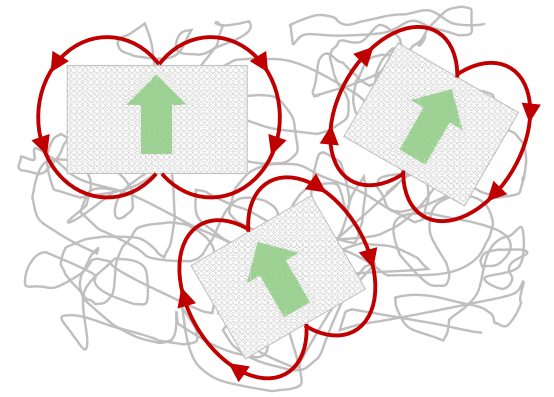

(d)

Figure 3.6: The (a)(b) Preisach Distribution and (c)(d) microstructure of (a) (c) BTA and (b)(d) P(VDF-TrFE).

associated to the coercive fields of the hysterons which is largely determined by their size.

This relation was confirmed by a simplified version of the electrostatic Monte Carlo model in Section 3.3.3. This deterministic version of the model calculates the PD in a way similar to experiments: the applied field is increased stepwise and the hysterons that switch each step correspond to the PD at that point of the Preisach plane. When the hysterons are far apart, they don't feel each other's fields and we obtain a narrow distribution as in $\mathrm{P}(\mathrm{VDF}-\mathrm{TrFE})$. When the hysterons are put closer together, the distribution broadens along the $E_{i}$ axis and becomes rounder like in BTA.

The Preisach model is thus not only a mathematical construct but a direct consequence of the morphology of the material. The broadness of the PD is caused by the microscopic disorder in terms of hysteron size and the interactions between them. The Gaussian shape of the PD reflects the Gaussian 
distribution of hysteron sizes, which for the BTA subcolumns and P(VDFTrFE) crystallites seems a reasonable assumption. This relation between PD and morphology can also be extended to inorganic ferroelectrics. These are usually polycrystalline with closely packed (and thus highly interacting) grains of varying size. One would therefore expect a round distribution, as has been observed in the few cases where a 2D measurement of the PD was performed $[141,150]$.

\section{Applications of the Preisach model}

We can now use the Preisach model to provide a physical basis for the switching time distribution in the NLS model. Using a special pulse sequence, we can selectively measure the switching time as a function of position on the Preisach plane $\tau(U, V)=\tau\left(E_{c}, E_{i}\right)$. The exponential dependence that is found can be explained with the theory of TA-NLS, as shown by the fit in Fig. 3.7b. Starting from Eq. (3.3), substituting Eq. (3.4) for the coercive field $E_{c}$, and adding the local field $E_{i}$ to the applied field, we get:

$$
\tau\left(E_{c}, E_{i}\right)=t_{\min }+\frac{1}{\nu_{0}} \exp \left(t_{1} \frac{E_{c, \text { in }}-E_{\text {app }}+E_{i}}{E_{c, \text { in }}-E_{c}}\right),
$$

where $t_{1}=\log \left(\nu_{0} t_{\exp } / \log 2\right)$ and $E_{c, \text { in }}=w_{b} / P_{s}$ have been used for simplicity. A minimal switching time $t_{\min }$ is included to prevent the hysterons close to the origin from switching unphysically fast, which might be associated with the time that is required for the physical rotation of the dipoles. Note that it is important that the applied field for measuring the switching time remains constant, as we have seen in Section 3.2.4 that the critical domain size changes with applied field.

The Preisach distribution gives us the spread in $E_{c}$ and $E_{i}$, the double Gaussian $N\left(E_{c}, E_{i}\right)=N\left(E_{c}\right) N\left(E_{i}\right)$ of Fig. 3.7a. We can convolute it with the switching time in Eq. (3.10) to obtain the switching time distribution. This corresponds to a transformation of the probability density according to

$$
N(\tau)=\int_{-\infty}^{\infty} N\left(E_{c}\right) N\left(E_{i}\left[\tau, E_{c}\right]\right)\left|\frac{\delta E_{i}}{\delta \tau}\right|_{\left(\tau, E_{c}\right)} d E_{c} .
$$

With this approach we find switching time distributions in BTA and P(VDFTrFE) that are close to lognormal, as shown in Fig. 3.7c. The distribution found through this microscopic Preisach method fits well with the switching kinetics obtained through macroscopic measurements. This further confirms the validity of our Preisach model and we thus have a full and consistent description of the macroscopic ferroelectric device in terms of material microstructure and disorder. Note that the above derivation is slightly different from that in Paper [2], where an error was made in convoluting the PD with the switching time. The final switching time distribution does not change much however. 


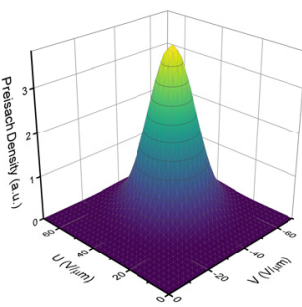

(a)

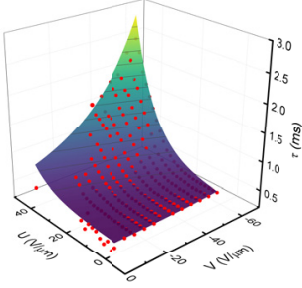

(b)

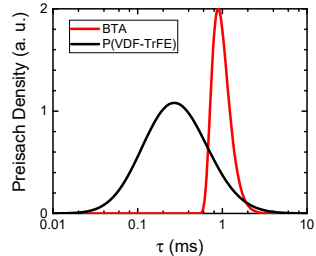

(c)

Figure 3.7: (a) The Preisach Distribution is convoluted with (b) the switching time as function of Preisach coordinates, where the dots are the experimental data and the surface a fit to Eq. (3.10) (here shown for BTA). This convolution gives (c) the macroscopic switching time distribution.

While the PD is a useful tool to model a material, it also has direct consequences for ferroelectric memory applications. In particular, disordered materials with a broad PD could be used for creating multibit memories. Intermediate states, corresponding to a PD that is partially poled, can be used to store data rather than just the binary up and down $[146,147,151]$. The more disordered a material is, the broader the PD, and the more bits you can store in a single memory cell. While materials with a round PD are limited to non-binary storage, the narrow shape of the PD in $\mathrm{P}(\mathrm{VDF}-\mathrm{TrFE})$ allows for the construction of a multi-bit memory with binary readout [146]. Due to the low remanent polarization of these intermediate states, the depolarization field is small and good retention can be obtained [147, 151].

\subsection{Monte Carlo Models}

\subsubsection{Introduction}

In the previous section we have outlined a variety of different models that analytically describe ferroelectrics either macroscopically or through statistical treatment of microscopic properties. The Monte Carlo models that we will discuss in this section are more microscopic and numerical in nature. They describe the material from the viewpoint of individual elemental units and their interactions, which for ferroelectrics are generally the electric dipoles and their electrostatic interaction.

Monte Carlo (MC) models get their name from their dependence on randomness that they share with the famous Monte Carlo casinos. They are widely used for simulations of many different kinds of physical systems, but are more broadly applicable to virtually any system that has a probabilistic interpretation. The main idea of MC is that by repeated sampling from some probability distribution, the samples will eventually reproduce this distribu- 
tion. This numerical sampling is often much more efficient than trying to calculate the correct distribution directly.

For the case of ferroic materials, the Ising model is a good starting point for a MC simulation. As is often the case, it was initially developed for ferromagnets, but can easily be applied to ferroelectrics as well. In the simplest $1 \mathrm{D}$ case, the Ising model considers a chain of dipoles $\mu_{i}$ that are either up or down $(\mu= \pm 1)$ and interact through nearest neighbor interaction only. The energy of this system is given by

$$
U=-\Sigma_{\langle i, j\rangle} J_{i j} \mu_{i} \mu_{j}-\Sigma_{i} E_{i} \mu_{i}
$$

where the first sum runs only over nearest neighbors and $E_{i}$ is the applied field at $\mu_{i}$. The interaction energy $J_{i j}$ determines whether neighboring dipoles tend to align parallel $\left(J_{i j}>0\right.$, ferroelectricity) or anti-parallel $\left(J_{i j}<0\right.$, antiferroelectricity).

While this simple Ising model can be solved analytically for a constant $J_{i j}$ and regular lattices in 1D and 2D (the latter only without applied field), numerical methods such as MC are necessary for more complicated geometries and interaction energies. In Ising MC models, we start with a given configuration of dipoles, or state, and then start flipping dipoles one by one according to the following algorithm:

1. Randomly pick one of the dipoles $i$ to flip.

2. Calculate the energy difference $\Delta U_{a, b}$ between the current state $a$ and the new state $b$ with $i$ flipped.

3. Calculate the transition probability $p_{a, b}\left(\Delta U_{a, b}\right)$.

4. Generate a uniformly distributed random number $0<\rho<1$, if $p>\rho$ then flip dipole $i$, otherwise retain the current state.

This continues until some stop criterion is met, at which point the equilibrium state of the system has hopefully been reached and the desired properties of the system (e.g. polarization) can be determined. The crucial part of this algorithm is the transition probability, which should ensure that the system moves towards equilibrium. The acceptance probability should therefore follow the principle of detailed balance: $p_{a, b} / p_{b, a}=P_{\text {eq,a }} / P_{\text {eq,b }}=\exp \left(-\Delta U_{a, b} / k_{B} T\right)$, where $P_{\text {eq }}$ is the occupational probability of the state in equilibrium. One of the simplest methods fulfilling this requirement is the Metropolis algorithm:

$$
p_{a, b}= \begin{cases}\exp \left(-\Delta U_{a, b} / k_{B} T\right), & \text { if } \Delta U_{a, b}>0 \\ 1, & \text { otherwise }\end{cases}
$$

While the Metropolis algorithm can be used in Monte Carlo simulations on ferroelectrics to obtain for example domain structures, it is limited to static 
equilibrium states. It can also be rather slow because many flipping attempts may get rejected. Instead, for fast simulation of non-equilibrium kinetics, a kinetic Monte Carlo (kMC) algorithm is used [152]. It starts by calculating the flipping rates $\nu_{i}=\nu_{0} p_{i}$ of all dipoles in the starting configuration, where $\nu_{0}$ is the so-called attempt frequency, as well as the total rate $\mathcal{V}=\Sigma_{i} \nu_{i}$. Then at each step:

1. Pick a dipole to flip using a uniformly distributed random number weighted by the flipping rate $\nu_{i}$.

2. Add $\Delta t=\log (\rho) / \mathcal{V}$ to the simulation time, where $0<\rho<1$ is again a uniformly distributed random number.

3. Flip the dipole and update all flipping rates.

While this algorithm avoids discarded attempts and can simulate kinetic processes, it requires one to keep track of all transition rates, which can be computationally expensive for large systems with many possible transitions.

\subsubsection{Monte Carlo Simulations on Ferroelectrics}

Applying Monte Carlo methods to ferroelectrics started in the early nineties [153]. These early models were restricted to simple 2D Ising systems with regular square lattices and constant nearest-neighbour-only interaction energies $J_{i j}$. Later models introduced more energy terms to the Hamiltonian, such as the depolarization field, domain wall energies, and mechanical strain [154, 155]. The simple interaction $J_{i j}$ could be replaced by the correct electrostatic expression:

$$
U_{i j}=\frac{1}{4 \pi \epsilon_{r} \epsilon_{0} r_{i j}^{3}} \vec{\mu}_{i} \cdot\left[\vec{\mu}_{j}-3 \hat{r_{i j}}\left(\vec{\mu}_{j} \cdot \hat{r_{i j}}\right)\right],
$$

where $\overrightarrow{r_{i j}}=r_{i j} \hat{i_{j j}}$ is the vector connecting the dipoles $i$ and $j$, which are not necessarily nearest neighbors. The long-range nature of this interaction means that it often needs to be cut-off after a certain range for computational efficiency, using methods such as Ewald summation or a reaction field to correct for this cut-off [156].

These models were mainly applied to the ferroelectric perovskites, and in particular BTO, to investigate static properties such as polarization, domain (wall) configurations, and dielectric anomalies. The effects of for example grain sizes, film thickness, and defects on those properties was studied as well $[155,157,158]$. More fundamentally, similar MC models were used to predict the emergence of ferroelectricity in polar discotics [159-161].

The studies mentioned above use static MC methods to calculate static properties such as domain configurations. While hysteresis loops are sometimes simulated as well, these have limited relevance because simulation steps can not directly be correlated to a real time (and similar to the experimental 
case, a hysteresis loop only has meaning if the sweep frequency is reported) $[154,157]$. Kinetic MC methods are therefore necessary to meaningfully calculate hysteresis loops. Here, the work of the group of Kliem has been pioneering.

Spanning two decades and many publications, the group of Kliem developed a realistic kMC model for solid dielectrics including ferroelectrics. Important characteristics of their model are the inclusion of metal contacts and induced dipoles on top of the permanent dipoles. They applied their model on different material systems, including BTO and PVDF [162], and were the first to extend $\mathrm{MC}$ simulations to three dimensions. They have investigated numerous different aspects of ferroelectrics, such as switching kinetics [163], domain wall motion [164], and the effect of thermal vibrations [165].

\subsubsection{Kinetic Monte Carlo Simulations on BTA}

We have developed a $\mathrm{kMC}$ model to describe the organic ferroelectric BTA in Paper [3]. While similar to the work of Kliem, it was developed independently and distinguishes itself by being able to account for unique and disordered 3D morphologies like that of BTA. A full description of the model can be found in Paper [3], but we will briefly introduce it here.

The model starts with the disordered 3D morphology of BTA in Fig. 2.15. From this morphology we get the positions of all the dipoles, which remain fixed during the simulation. The only thing that changes throughout the simulation is that the dipoles flip between pointing up or down. The probabilities of these flips are calculated as detailed in the previous sections, using the full dipole interaction of Eq. (3.14) within an interaction range. Dipoles outside of this interaction range are accounted for through the reaction field method [156]. Periodic boundary conditions are applied in-plane, and the top and bottom electrode are treated as perfect electrostatic mirrors. As in the work of Kliem, we take into account induced dipoles as well, which are located at the same position as the permanent dipoles but are free to orient along the local field. All input parameters can be found in Paper [3].

\subsubsection{Retention}

We first investigate the behavior of the system without applied field, i.e. spontaneous depolarization. We find that the material depolarizes according to the stretched exponential in Fig. 2.10b, as is also found experimentally [5, 41]. The depolarization is thermally activated, with the retention times in Fig. 3.8a following Eq. (3.3) of the TA-NLS theory (with $E_{\text {app }}=0$ ). A good match with the experimental retention times is obtained, and the energy barrier for depolarization $W_{b}=0.75 \mathrm{eV}$ matches the experimental value as well.

By letting the simulations run until the material has depolarized completely, we can obtain information about the ground state. We find that the 
individual columns stay polarized even though the net polarization is zero. When the material is viewed from the top, the $2 \mathrm{D}$ domain structure that appears has stripe domains that indicate (frustrated) antiferroelectricity. The dipolar interactions between neighboring columns favor an antiparallel alignment, and the hexagonal packing of the columns leads to a frustrated state. Detailed analysis of the correlations between columns show that the ground state is indeed antiferroelectric. This is in agreement with observations in literature based on X-ray diffraction [166, 167]. While the ground state of BTA is thus antiferroelectric, this is of little relevance during experiments. Once the material is fully poled, it will remain so due to the high energy barrier for depolarization and the corresponding long retention times of several months.

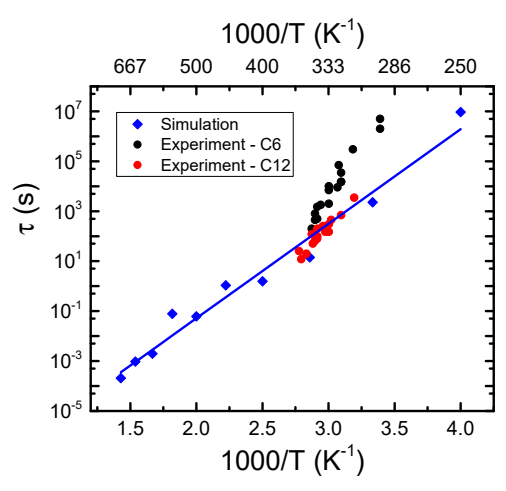

(a)

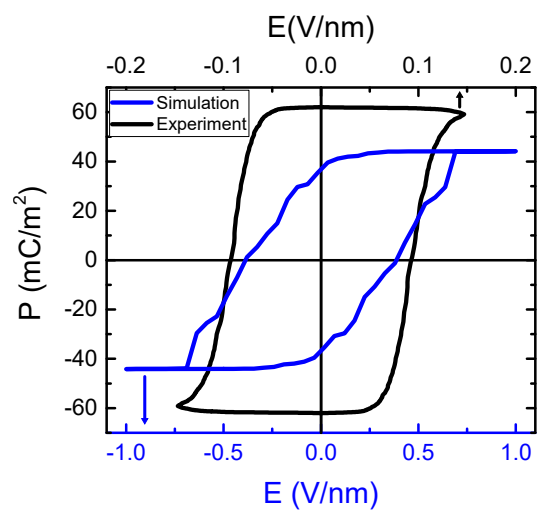

(b)

Figure 3.8: Results of the kMC simulations on BTA for the (a) retention time and (b) hysteresis loops at $325 \mathrm{~K}$ and $25 \mathrm{~Hz}$.

\subsubsection{Hysteresis Loops}

We now turn to simulating field-driven polarization switching, specifically the hysteresis loop. We find in Fig. 3.8b that the material indeed shows hysteresis. There is a reasonable agreement with the experimental loop, although the coercive field is a factor 4 too high. Too high coercive fields are a common result in simulations and are generally the result of the models not being able to capture the fully extrinsic nature of the polarization switching in experiments [50]. It should be noted that this hysteresis loop was obtained with input parameters based on previous theoretical and experimental results and that no fitting parameters were used.

If we take a closer look at how the polarization within a column reverses, we notice that there are two possible pathways, indicated in Fig. 3.9. The dipoles can either reverse only their z-component, or flip fully. Note that for the $\mathrm{z}$-flip the helicity of the helix reverses. In the simulations we can restrict 
the material to one specific flipping mode and compare the results. Comparing both hysteresis loops for BTA in Fig. 3.9b, we see that the z-flip has a smaller coercive field and is thus expected to dominate the switching kinetics. This is consistent with MD simulations where only the z-flip is observed, as discussed in more detail in Section 3.4.

The hysteresis loop of the full flip shows a plateau at $\pm 2 \mathrm{~V} / \mathrm{nm}$. Closer inspection of this plateau reveals that it corresponds to an intermediate state in which two of the three dipole helices have reversed, as shown in Fig. 3.9a. In the z-flip mode, all three helices flip simultaneously and this shoulder is thus not observed. The fact that the shoulder is not observed experimentally is further proof that the z-flip is normally dominant in BTA. In the case of BTTTA the situation is slightly different (see Fig. 2.18c and Section 4.2.3). This material has a helical pitch of 9 molecules compared to the 6 of BTA, which means that reversing only the z-component of the dipoles does not lead to a new hydrogen-bonded network. BTTTA is thus restricted to the full flip, which can explain its relatively high coercive field [7].

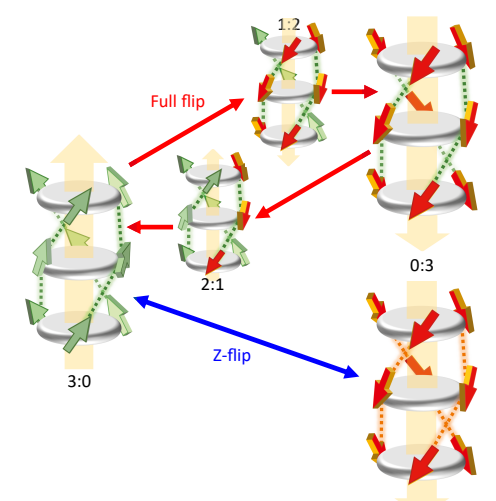

(a)

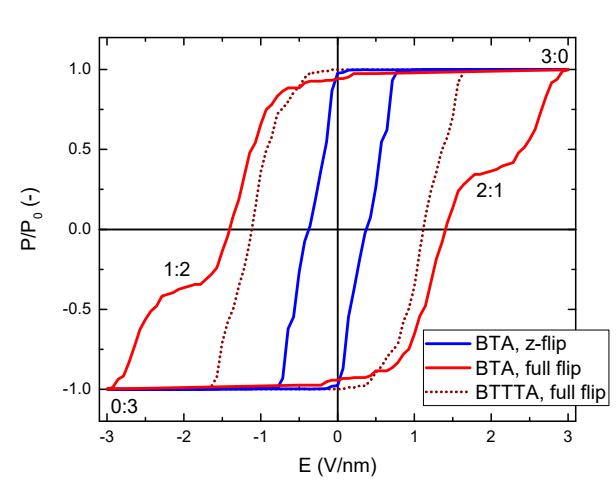

(b)

Figure 3.9: (a) The two different switching modes and (b) the corresponding hysteresis loops in BTA and BTTTA at $325 \mathrm{~K}$ and $25 \mathrm{~Hz}$.

\subsubsection{The Effect of Disorder}

One of the strengths of our model is that it can take into account the disordered morphology of BTA, with its defects that divide the columns into subcolumns. By varying the number of defects, we can investigate the effect this disorder has on the ferroelectric properties. In Fig. 3.10 we see that when the disorder increases, the retention time decreases dramatically. On the other hand, the hysteresis loop and especially the coercive field barely 
changes. The loops only become more slanted with increasing disorder as can be expected from the Preisach model of Section 3.2.5.3.

This suggests that there is a difference in the switching kinetics between spontaneous and field-driven polarization switching. Indeed, when we visually inspect the nucleation and propagation of the polarization reversal, we notice a difference in the nucleation process. For field-driven polarization switching such as in hysteresis loops, nucleation occurs at the electrodes, whereas for spontaneous depolarization it occurs at defects. By reducing the number of defects, the depolarization can thus be suppressed without much effecting the coercive field. This is a relevant insight for designing ferroelectric memories out of these materials. While this result has been obtained specifically for BTA, this difference in kinetics could be relevant in other ferroelectrics as well.

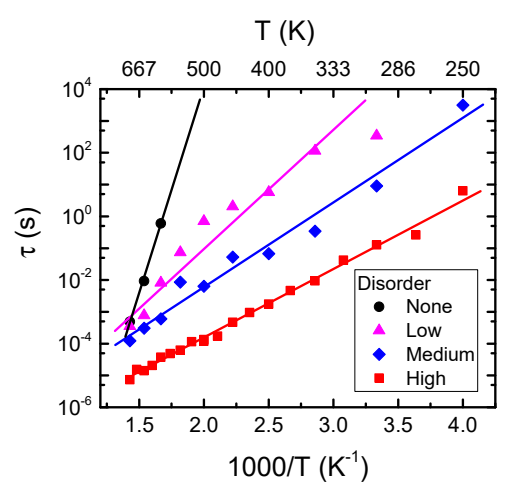

(a)

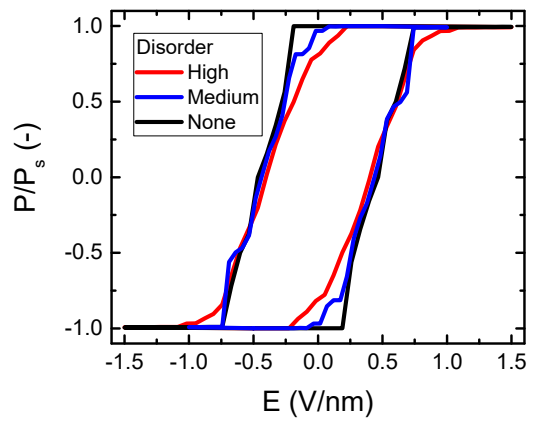

(b)

Figure 3.10: The effect of disorder in BTA on (a) the retention time and (b) hysteresis loops at $325 \mathrm{~K}$ and $25 \mathrm{~Hz}$.

\subsubsection{Switching Transients}

The final ferroelectric experiment to simulate using our model is the switching transient, which is done in Paper [4]. The shape of the simulated transients roughly follows the (dispersive) KAI equations discussed in Section 3.2. More interesting is the dependence of the switching time on applied field, which is expected to follow the Merz law of Section 3.2.2. In Fig. 3.11 the simulated switching times are compared to those found in experiments.

We find that while the simulations do follow the Merz law as in the experiments, the switching is either too fast at high fields, or too dispersive at low field. Furthermore, the field dependence is much stronger in the simulations. We have ruled out several possible explanations for this discrepancy, the details of which can be found in Paper [4]. In short, introducing different types 


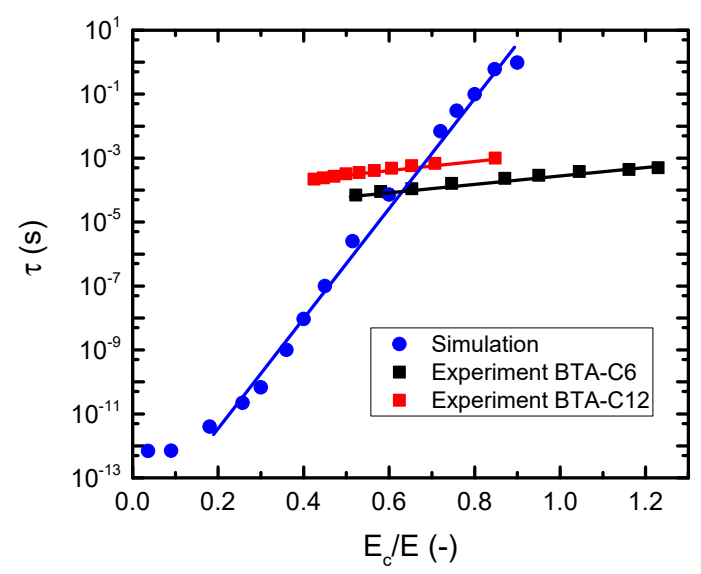

Figure 3.11: The simulated and experimental switching time as a function of the reciprocal applied field, normalized to the coercive field.

of defects in the simulations cannot remove the discrepancy. The issue can also not be solved by the inclusion of 'dead' interfacial layers, being highly disordered or even non-switching. Similarly, interpretations of the experiments in terms of the 'leaky layer' model by von Seggern and Fedosov or in terms of system RC times are ruled out [168].

We therefore speculate that the discrepancy could be caused by a finite dipole switching time in the actual material. In reality, flipping a dipole requires a physical rotation of the amide group, which is sterically hindered and takes a certain time. In our simulations however, dipoles flip instantly. Unfortunately, incorporating a finite flipping time into the $\mathrm{kMC}$ model is not possible, as this would require multiple events to happen at the same time. One could expect that having such a flipping time would not only reduce the values but also the field-dependence of the switching time, as the switching would be slowed down especially at high fields. This flipping time would have only a minor influence on (slow) hysteresis loops and retention because these happen at longer time scales.

Regardless of the discrepancy with the experiments, it is important to emphasize that the simulations follow the Merz law. As discussed in Section 3.2.2, the Merz law is generally associated with domain walls and defects. We show that Merz behavior emerges in a simple system of purely electrostatically interacting dipoles, without explicitly considering domain wall or depolarization energies. It is even obtained in a system without any defects (an infinitely long column) that could provide pinning or nucleation sites. This is in agreement with results from the group of Kliem, who also found the Merz law with their 
similar model, albeit over a smaller range of fields [164, 165]. These results indicate that the Merz law is even more general than previously assumed.

We can get a better understanding of the emergence of Merz behavior by again manually inspecting the dipole switching. A typical nucleation process at a bottom contact is presented in Fig. 3.12a. Looking at the energy as a function of time, we see a clear maximum that corresponds to the critical nucleus energy $U^{*}$ and size $N^{*}$. We analyze such nucleation events for ensembles of columns at different temperatures and fields to extract the nucleus size and energy as a function of applied field in Figs. 3.12b and 3.12c. Both the nucleus size and energy are found to be proportional to $1 / E$. At higher temperatures we see deviations from this behavior because the higher thermal energy makes nonoptimal nucleation pathways available.

The Merz law thus emerges due to the $1 / E$ field dependence of the nucleation mechanism and specifically the nucleus size. This dependence can be rationalized using a qualitative argument based on a simple Ising model, as presented in Paper [4]. This intuitive argument leads to a linear approximation of the nucleus energy as a function of both size and field, which gives the $1 / E$ dependence of the critical nucleus size. This field-dependent nucleation volume was used in Section 3.2.4 to develop the thermally activated Merz theory, which gives a coherent description of both experiments and simulations.

\subsection{Molecular Dynamics}

Molecular Dynamics (MD) is an atomistic level method to calculate the physical movements of atoms. It treats (groups of) atoms as classical particles that interact through approximated forces, and numerically solves the resultant Newtonian equations of motion. It can describe ground state properties, such as the optimal configuration of a supramolecular polymer. But as the name suggests it is mainly suited for simulations of dynamics, and as such can be used to obtain thermodynamic properties and time-resolved responses to external stimuli. This makes MD well suited to simulate polarization switching in ferroelectrics.

The key point in running useful MD simulations is the choice of the interatomic potentials or force fields. These potentials should capture all the relevant physics that is involved in the interaction between the atoms. They can be rather simple and semi-empirical, such as the well-known LennardJones potential, or be derived from ab initio calculations. A balance between accuracy and computational efficiency will thus have to made depending on the application. The chosen interatomic potential will also be highly dependent on the material and the particular properties of interest. 

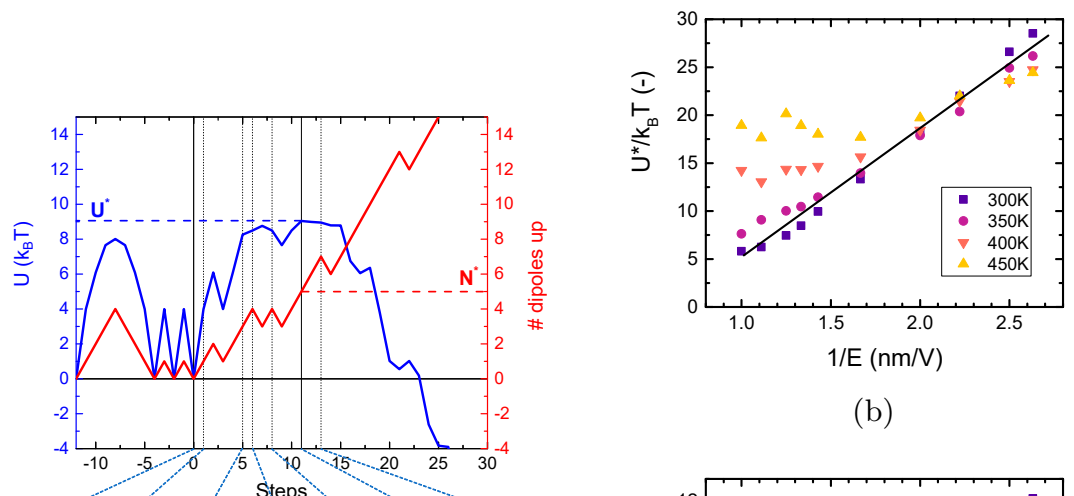

(b)

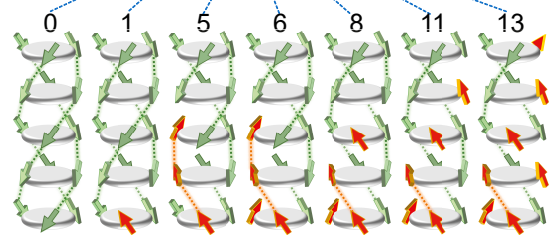

(a)

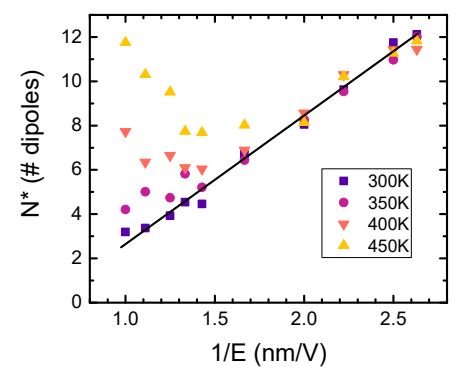

(c)

Figure 3.12: (a) A typical nucleation process in a single column of BTA. The energy, polarization, and dipole configuration are shown throughout the nucleation, where steps are defined relative to the start of the nucleation at step 0 . The critical nucleus size and energy, occurring at step 11, are indicated. By averaging over multiple nucleation events like these, the (b) nucleus energy and (c) size as a function of reciprocal applied field are determined.

\subsubsection{MD on Ferroelectrics}

To perform MD simulations on ferroelectrics, it is thus imperative to construct the right interatomic potentials. Due to the large variety of ferroelectric materials and differing origins of their ferroelectricity, there is no one-size-fits-all solution. We will not go into detail on the different approaches used in the literature and instead just highlight some important contributions and results. Detailed overviews of MD on (ceramic) ferroelectrics can be found in several reviews [50, 169-172].

Early MD work in the late nineties used the shell model to calculate interatomic potentials and focused on describing static properties such as ferroelectric phase transitions [173]. Later, models were developed that could also describe polarization switching [174]. An important milestone is the development of the feram code by Nishimatsu and coworkers based on an effective Hamiltonian approach [175-177]. Feram uses a Fast Fourier Trans- 
form technique to surpass the bottleneck of calculating the long-range dipole interactions, greatly increasing computational efficiency. This freely available code has been used by many groups to study a large variety of static and dynamic ferroelectric properties in perovskites [172].

When it comes to simulating polarization switching and domain wall motion, the group of Rappe developed an interatomic potential based on the bond valance model that accurately describes perovskites [178]. This model is used to perform detailed simulations of the nucleation and domain wall motion in PZT and the results can be described by the Merz law [122, 123]. Their work is of multi-scale nature, as the input to the MD simulations come from first principle calculations and the results are used for larger scale MC and analytical models.

\subsubsection{MD on BTA}

The methodologies above have been developed specifically for inorganic ferroelectrics. Molecular systems like BTA require rather different approaches. BTA specifically has been studied intensively by theoretical chemists with a variety of MD and DFT techniques, in particular because of its interesting self-assembly properties [88, 179].

MD on BTA has mainly been done by the group of Balasubramanian [179]. They have performed extensive investigations of the cooperative self-assembly of BTA, and found for example that the 2:1 state, with one of the three helices pointing antiparallel, is more stable to the 3:0 state [87, 180]. Particularly relevant is also the work of Bejagam et al. [181], which investigated the effect of an applied field on a column of BTA, effectively simulating a polarization switching experiment. They found that the dipoles reverse by flipping only their z-component, thereby reversing the handedness of the triple helix.

Their results correspond well to those from our kMC model as well as our own MD simulations, as discussed in Section 3.3.3 and Paper [3]. While the $2: 1$ state is a local minimum, it is normally not accessed during polarization switching. We also find that the z-flip is preferred, and from the MD simulations we get the flipping scheme in Fig. 3.13. When applying a negative electric field on a fully polarized 3:0 column, the dipoles will flip through their z-component only. Even when starting from the 2:1 state, with one helix already pointing down, a negative electric field will still cause the remaining 2 helices to only flip their z-component, causing a reversal of the handedness of the column.

We have used MD to support our experimental work in a few more studies on BTA. In an investigation of the effect of (branched) tail substitution, the simulated switching times of various BTA homologues follow the Merz law [7]. The simulations further elucidate how morphological disorder plays a key role in the ferroelectric properties of a material. The importance of disorder is also evident when simulating the piezoelectric coefficient of BTA [59]. With MD 


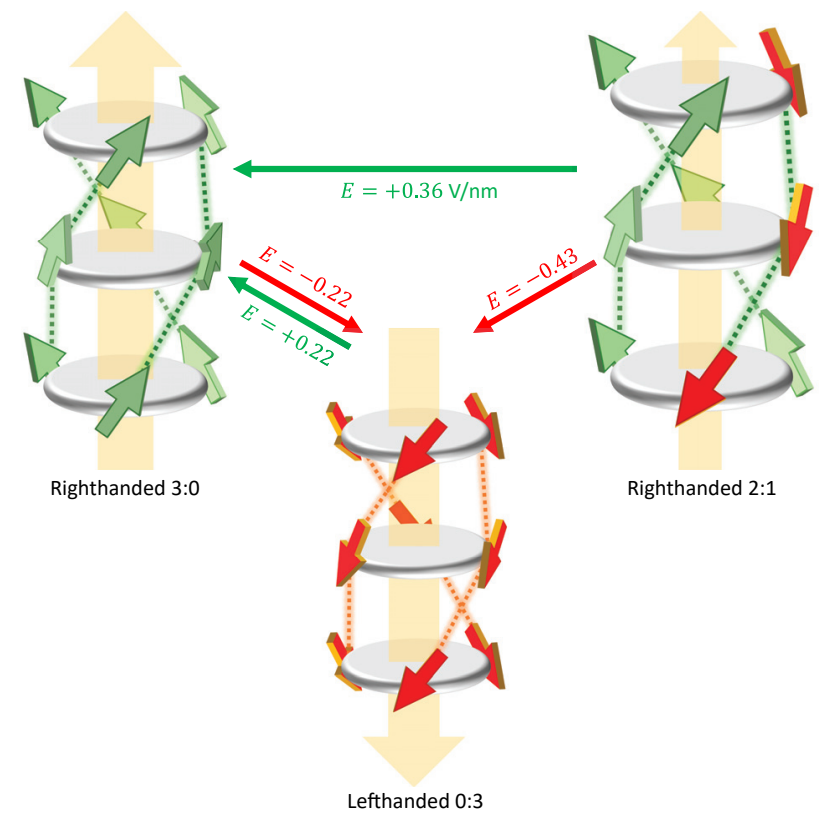

Figure 3.13: The different states and flipping modes as calculated by MD, with the electric field necessary to cause flipping indicated.

and DFT, a positive piezoelectric coefficient $d_{33}$ is obtained, whereas experimentally an anomalous negative $d_{33}$ is found. This can be explained by the disordered morphology of BTA, like in $\mathrm{P}(\mathrm{VDF}-\mathrm{TrFE})$, where the macroscopic dimensional effect determines the piezoelectric response.

\subsection{First Principle Theory}

\subsubsection{Density Functional Theory}

Finally, we have arrived at the highest level of theory, that of first principle or ab initio calculations. These methods try to directly solve the Schrödinger equation for the electrons and nuclei in an external potential without any experimental input. Because this quickly becomes unmanageable due to the many interacting electrons, approximations are necessary. The BornOppenheimer approximation states that the nuclei can be treated classically because of their comparatively large mass. Hohenberg and Kohn subsequently realized that all ground state properties of a system are a functional of just the electron density, circumventing the need to calculate the full many-body electronic wave function. Kohn and Sham then provided a workable technique for solving for the electron density by using independent electrons embedded in 
an effective potential. This is the basis of Density Functional Theory (DFT), which underlies most first principle calculations [182].

One of the main challenges of DFT is to find the exchange correlation term of the effective potential. One of the most basic approaches is the Local Density Approximation, which assumes that the exchange correlation potential at a point is only dependent on the electron density at that specific point. This approximation works surprisingly well and is therefore often used, although more accurate methods that for example consider density gradients are available. Another important choice is that of the basis set for representing the independent electron wavefunctions, for which plane waves are a common choice.

As with MD, the choice of method is highly dependent on the material and properties of interest. Computational efficiency is also a major concern, which is especially true for ferroelectric perovskites with computationally unfriendly atoms such as oxygen and transition metals. We will again not go into detail about the different methodologies used for DFT calculations, and only discuss the most important contributions here. Interested readers are referred to several comprehensive reviews [50, 102, 171, 183, 184].

Initial efforts to describe ferroelectrics using first principle methods were restricted to structural properties of the different phases of ferroelectric perovskites. The first example of this was a study by Cohen and Karkauer in 1990, which demonstrated the stability of the ferroelectric phase in $\mathrm{BaTiO}_{3}$ [185]. Due to the ambiguous definition of polarization, discussed in Section 2.3.1, it was not possible to calculate the spontaneous polarization until the development of the modern theory of polarization [25, 26]. Quickly after that, Resta et al. were the first to apply this novel theory to calculate the spontaneous polarization of $\mathrm{KNbO}_{3}$, which agreed well with the experimental value [186]. The modern theory of polarization also allowed the first correct first principle calculation of piezoelectric coefficients in a ferroelectric [187].

DFT calculations typically use periodic boundary conditions in all three dimensions. While this is relevant for bulk properties, in applications thin films are often used. The properties of these thin films can differ significantly from the bulk material $[58,102]$. Periodically repeated slabs can be used to emulate thin films, but this leads to problems with artificial electric fields arising in the vacuum due to the polarization surface charges. Furthermore, the interface between the ferroelectric and the metallic contacts requires proper treatment. A first realistic treatment of thin films was presented by Junqera and Ghosez, who showed that $\mathrm{BaTiO}_{3}$ thin films lose their ferroelectricity below a certain critical thickness due to the depolarization field [188]. More recently, Stengel et al. stressed the role of interfacial chemistry in a paper that presented a general methodology for calculating thin films with realistic electrodes [189].

The exponential increase in computational power has made it possible to use DFT on larger and more complex systems. For example, it has allowed the 
investigation of more complex domain structures rather than single domains. It was shown that in ultrathin $\mathrm{BaTiO}_{3}$ films, a polydomain structure reminiscent of magnetic closure domains arises $[190,191]$. This leads to a reduction in the depolarization field, and the persistence of ferroelectricity below the critical thickness previously found. DFT is now also routinely used to verify ferroelectricity in newly discovered (classes of) materials, such as the organic crystals [78, 192] and polymers [193].

\subsubsection{DFT on BTA}

The computational complexity of DFT complicate its application to supramolecular systems. For example, a single column of ten BTA-C6 molecules already consists of close to 1000 atoms. Therefore, the side chains are often ignored by simulating BTA-C1 instead. This greatly reduces the computational complexity, while preserving the most important characteristics of BTA.

Using this approximation, the self-assembly properties of BTA have been investigated by several groups $[89,94,194,195]$. In particular the cooperativity of this process has been of great interest. It was found that upon elongation of the column, each subsequent molecule added would lower the energy more than the previous. This was accompanied by a decrease in hydrogen bond length and band gap, as well as an increase in the dihedral angle of the amide groups. Most importantly, the dipole moment per monomer was also found to increase with column length, leading to a large spontaneous polarization. This polarization was given as the reason for the $2: 1$ state being more stable than the 3:0 state, as was also found with the MD simulations discussed in the previous Section $3.4[87,180,181]$.

We use these DFT results to obtain our input parameters for the kMC model of Section 3.3.3. The fact that the dipole moment increases as a function of column length suggests that there is an induced electronic polarization on top of the polarization from the permanent dipole moments of the amide groups. We calibrate the permanent dipole moment and the polarizability in the $\mathrm{kMC}$ model to this permanent and induced polarization from the DFT calculations. Finally, DFT calculations were also used to calculate the piezoelectric coefficient of a single stack of BTA [7]. This nanoscopic $d_{33}$ was found to be positive, contrary to the macroscopic experimental result. 


\section{Chapter 4}

\section{Semiconducting Ferroelectrics}

\subsection{Introduction: Ferroelectric Memories}

The models presented in the previous chapter are crucial in understanding ferroelectric materials. This understanding is in turn crucial for designing materials that can be used in ferroelectric device applications. In this chapter, we focus on one of the most basic of ferroelectric devices: the memory. In particular, we will discuss how the combination of ferroelectric and semiconducting properties leads to interesting new physics as well as possible memory device applications.

In this introductory section, we will briefly discuss the basic principles of ferroelectric memories. This includes the readout problem, which can be solved by combining ferroelectricity with semiconductivity. In Section 4.2, we will introduce several different types of semiconducting ferroelectrics and the conductivity switching behavior that they exhibit. Finally, in Section 4.3 we will present a model to describe this conductivity switching.

Ferroelectrics have long been considered as an attractive candidate for non-volatile memory applications due to their bistable polarization that can easily be controlled by an electric field. However, it wasn't until the late 90s that ferroelectric memories became commercially viable in the form of capacitor-based Ferroelectric Random Access Memories (FeRAM) [196, 197]. Due to several limitations such as scalability and stability, traditional ferroelectric memories are only a niche market nowadays, being outcompeted by flash memories for short term and magnetic memories for long term storage. Nevertheless, in the field of organic and flexible electronics ferroelectric memories are a promising option due to the absence of viable organic flash or magnetic memories $[74,75,198]$. 
One of the main issues with a ferroelectric capacitor memory is the readout. This is done by applying a voltage to the memory cell and measuring whether a switching current is flowing. The readout is thus destructive, and might require an additional write operation afterwards to restore the cell to its original state. This is particularly problematic for systems were fatigue is an issue, such as most perovskites, as the constant switching of the ferroelectric will degrade its performance. Scalability is another big problem. The switching current is proportional to the area of the device, so the smaller the memory cell, the harder it becomes to detect the switching current.

\subsection{Semiconducting Ferroelectrics}

Since there is no direct way of nondestructively reading out the polarization state of a ferroelectric, several indirect ways have been investigated. These methods use the coupling of ferroelectricity with other physical properties. A relatively recent development in this regard is the field of multiferroics, where multiple ferroic properties such as magnetism and ferroelectricity coexist. This is beyond the scope of this thesis however, and a recent review gives a good overview of the topic [11].

Here, we will focus on the interplay between ferroelectrics and semiconductors. The basic idea behind this is that the ferroelectric polarization will somehow affect the conductivity of the semiconductor. This allows the memory state of the ferroelectric to be read out simply through measuring the conductivity of the semiconductor, without affecting the ferroelectric polarization. This also solves the scalability problem as long as decent on/off ratios are obtained. We will first discuss the technique of combining a semiconducting with a ferroelectric material, after which we move to single materials that exhibit both properties.

\subsubsection{Combining Semiconductors with Ferroelectrics}

A straightforward way to integrate ferroelectrics with semiconductors is by replacing the gate dielectric in a classical Field Effect Transistor (FET) with a ferroelectric. This results in the ferroelectric FET (FeFET) shown in Fig. 4.1a. The ferroelectric takes over the role of the dielectric and depletes or accumulates charges in the semiconductor, thereby switching on or off the conduction from source to drain. It thus functions similar to a normal FET, with the difference that the ferroelectric will maintain its polarization when the voltage is removed, making it a non-volatile memory. Interested readers are referred the literature for extensive reviews on FeFETs based on both inorganic [196, 199] as well as organic materials [75].

The preferred device architecture for a memory device would be the crossbar array in Fig. 4.2 [200]. By addressing the right combination of bit and word line, the memory cell can be written or read. This simple architecture 


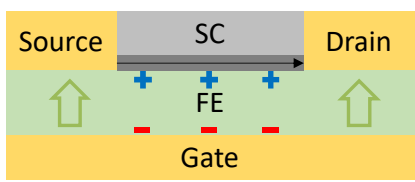

(a)

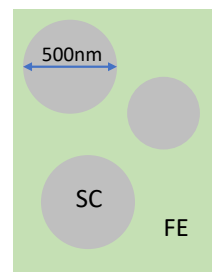

(b)

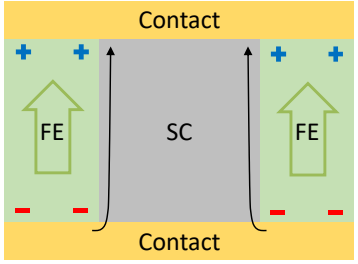

(c)

Figure 4.1: Two different types of memories that combine the functionality of semiconductors and ferroelectrics. (a) A FeFET, where the ferroelectric (FE) enhances or blocks conduction through the semiconductor (SC) channel (dark grey). A (b) top and (c) side view of a lateral ferroelectric diode obtained by mixing two organic polymers, where the ferroelectric controls the charge injection into the semiconductor. In both devices the current path is shown by a black arrow.

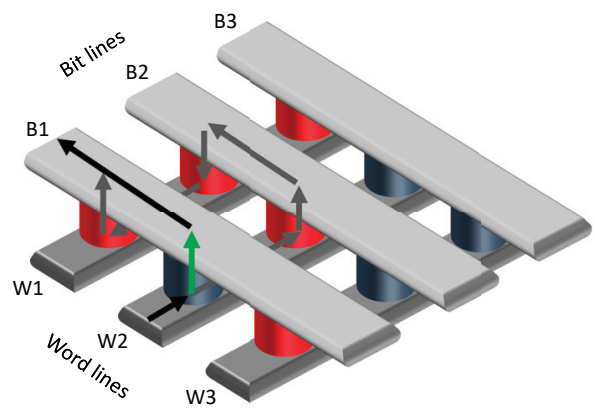

Figure 4.2: A crossbar memory, showing the attempted readout of cell W2B1 in black, with the desired current path in green and the sneak path in grey.

allows for easy fabrication of high-density memories. Unfortunately, the three terminal nature of FeFETs hinders their application in a crossbar array. Another issue is that of crosstalk, which is demonstrated in Fig. 4.2. When trying to read out a cell that is in its high resistance state, the cells surrounding it might provide a sneak path for the current that would incorrectly suggest that the targeted cell is in the low resistance state.

The problem of crosstalk can be solved by adding rectifying diodes to each memory cell, which block the sneak current path. This would add unwanted complexity to the fabrication process and it would therefore be preferable if this diode characteristic is inherent to the memory cell. This was first realized by Asadi et al. in the lateral heterostructure shown in Fig. 4.1c, instead of a vertical one as in FeFETs [12].

In these devices, the ferroelectric polymer $\mathrm{P}(\mathrm{VDF}-\mathrm{TrFE})$ is mixed with a semiconducting polymer such as poly(3-hexylthiophene) (P3HT). Due to 
spontaneous phase separation the morphology in Fig. 4.1b is obtained, with semiconducting columns in a ferroelectric matrix. The electrodes are chosen such that one side blocks injection of both types of charge carrier, while the other side is a Schottky diode. The injection barrier of this Schottky diode is modulated by the ferroelectric polarization, leading to the desired conductivity modulation as well as rectification.

The memory devices obtained in this manner show a good on/off ratio, cycling endurance, and retention, although the latter might be fundamentally limited by the device geometry $[74,75,198,201]$. The concept has also proven scalable, demonstrated by the construction of a 1 kbit array with a bit density up to $10 \mathrm{kbit} / \mathrm{cm}^{2}$ on flexible foils [202]. The exact operational mechanism of these switchable diodes has been investigated through numerical simulations. Initially, these simulations indicated that a large stray field between the polarization and compensating charges was responsible for modulating the injection barrier [203]. This was later refined to an operational mechanism like a vertical FET with charge transport mostly along the semiconductor/ferroelectric interface and tunneling injection [204].

A major challenge for these types of memories is the control over the morphology. The demixing of the polymers is a spontaneous process and therefore hard to control. This leads to the random distribution in both size and position of the semiconducting columns, which are fairly large on average $(\sim 500 \mathrm{~nm})$. Ideally one would like to have a uniform distribution of semiconducting columns with a small (tens of nanometer) and consistent size. While some control over the phase separation is possible through for example patterned substrates and blend ratios [205], full control over the morphology and thereby device performance remains a challenge.

\subsubsection{Inorganic Ferroelectric Semiconductors}

Instead of combining a semiconducting with a ferroelectric material, it would be easier to have a material that exhibits both properties at once. Unfortunately, it is difficult to obtain both (semi)conductivity and a stable polarization because the free charge carriers tend to compensate the polarization charges. Most classical ferroelectrics such as $\mathrm{BaTiO}_{3}$ are considered insulators or at least wide band gap semiconductors with band gaps above $3 \mathrm{eV}$. Nevertheless, the effect of the ferroelectric polarization on the semiconductive properties of these materials has been investigated.

Resistive switching was first demonstrated by Blom et al. [206] in a ferroelectric Schottky diode. They found that the polarization in $\mathrm{PbTiO}_{3}$ could modulate the depletion width of the Schottky Au electrode, leading to a switchable diode that could serve as a single material memory. However, the high resistivity of the material limits the on/off ratio of the very small readout current. Doping can increase the conductivity but reduces (the stability of) the polarization. Another way to increase the conductivity is to greatly 
reduce the thickness of the ferroelectric layer so that a tunneling junction is obtained. The tunneling barrier will again be modulated by the polarization, which is the basis for a Ferroelectric Tunnel Junction (FTJ) [207, 208]. Unfortunately, the fabrication of these device is very challenging due to the requirement of smooth ultrathin films.

The photovoltaic properties of ferroelectrics have been investigated since the 70s [10]. Remarkably, some ferroelectrics show a photovoltage that can be many times greater than the band gap of the material, in contrast to conventional photovoltaics. This has been ascribed to the Bulk Photovoltaic Effect (BPE), where the asymmetry of the ferroelectric polarization is responsible for the large photovoltage. The exact mechanism behind the BPV is still disputed, and various models exist [209-212]. This field of research has gained renewed interest after Choi et al. showed that the multiferroic $\mathrm{BiFeO}_{3}$ can behave as a switchable photodiode [213]. This has led to the fabrication of for example memories [214] and a high efficiency multilayer solar cell [215]. However, a better understanding of the BPE in different materials is necessary before commercially viable applications are possible.

\subsubsection{Organic Ferroelectric Semiconductors}

One of the great advantages of organic electronics over inorganics is the ability to easily modify the molecular structure of the active material with a near infinite selection of functional groups. This suggest a strategy for obtaining semiconducting ferroelectrics that is rather straightforward compared to inorganics: design a molecule that combines dipolar and semiconducting functional groups. This is exactly what we have done with the supramolecular discotics introduced in Section 2.5.3. The dipolar amide groups provide the ferroelectric functionality as in BTA, whereas the large $\pi$-conjugated cores make the materials semiconducting.

Similar materials that are polar but not ferroelectric (i.e. non-switchable) were first reported by Guilleme et al. [216]. They synthesized and characterized a compound based on subPc without switchable amide groups, but with a fixed dipole in the core of the molecule. The material is poled by applying an electric field while cooling from the isotropic liquid phase, resulting in a polar and semiconducting columnar liquid crystal. Recently, Zhang et al. measured the photovoltaic properties of a similar subPc compound and claimed to have observed a BPE [217]. While they do measure a photocurrent that reverses with the poling direction, some of their results are questionable. This includes the photovoltage increasing with thickness well beyond the penetration depth of the light, the absence of above bandgap photovoltages, and the observance of a photovoltage in devices that are poled with an AC field and therefore should be symmetric. Preliminary measurements of our own on the same compound do indeed suggest an asymmetric conductivity after DC 
poling, but extended characterization is necessary to confirm that this is due to the polarity of the material.

Designing and synthesizing organic semiconducting ferroelectrics is only a very recent development. So far, besides our own work, only two other groups have demonstrated these types of materials. The group of Funahashi has presented a class of smectic liquid crystals, whereby they mainly focused on the photovoltaic properties [218-220]. The group of Akutagawa has designed fluorescent materials that follow the same core-shell design principle as our work, also with amides as the dipolar moiety [221-224].

In our work, we have mainly focused on understanding the effect of the ferroelectric polarization on the conductivity of these materials. The three different materials in Fig. 2.18 exhibit different conductivity switching mechanisms, that will be discussed in the upcoming sections. We start with Injection Barrier Modulation (IBM) in $\alpha$-CBT, then discuss Bulk Conductivity Switching (BCS) in subPc-amide, and finish with the occurrence and applicability of both mechanisms in BTTTA.

\subsubsection{Injection Barrier Modulation}

The $\alpha$-CBT molecule, as presented in Fig. 2.18a, has a morphology that is similar to BTA with the formation of supramolecular columns that stack in a hexagonal liquid crystal [101]. Ferroelectric characterization shows that there is some dipolar hysteresis, but the loop shape is far from ideal. LIMM (see Section 2.4.6) shows that there are switchable dipoles only within $50 \mathrm{~nm}$ of the electrode. This means that the material is not really a bulk ferroelectric, although later experiments suggest that this might have been due to alignment issues [59].

The thin dipolar layer is however sufficient to modulate injection barriers in a way similar to the ferroelectric/semiconductor blends. The possible injection barrier modulation is estimated at $\Delta V \approx d P_{s} / \epsilon \approx 0.5 \mathrm{eV}$, where $d$ is the typical thickness of the injection layer. With a HOMO of $-5.7 \mathrm{eV}$, this suggests that hole injection from/into gold, with a work function around $-5 \mathrm{eV}$, could be modulated.

The IBM as shown Fig. 4.3a is indeed obtained in a device with symmetric $\mathrm{Au}$ electrodes. When the coercive field is passed, the device switches from an injection limited off state to a Space Charge Limited (SCLC) on state. It stays in this on state until the current direction is reversed, since holes should then be injected from the opposite electrode where the dipoles are blocking injection. This is the same behavior as observed in the ferroelectric/semiconductor blends. 


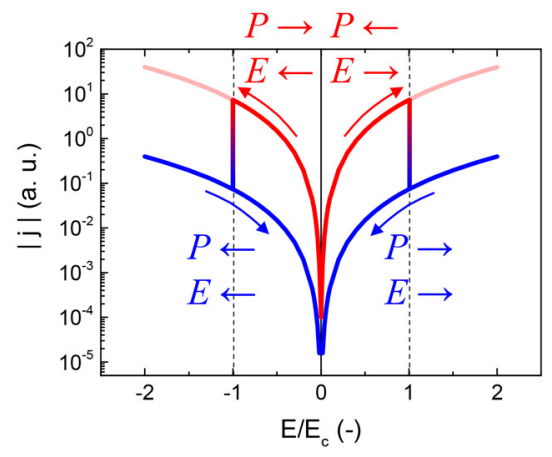

(a) Injection Barrier Modulation

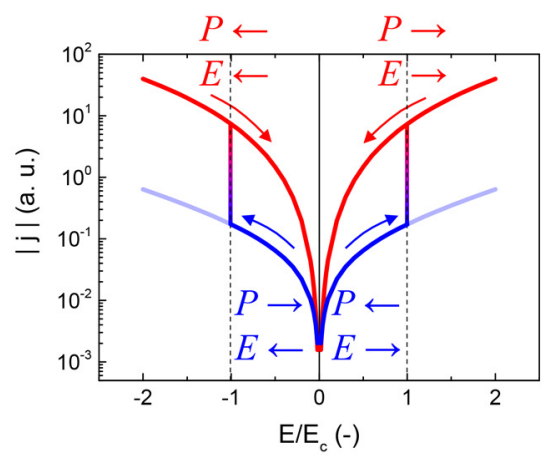

(b) Bulk Conductivity Switching

Figure 4.3: The two types of conductivity switching observed in organic semiconducting ferroelectrics, where the switching between the on (red) and off (blue) states happens at the coercive field and upon reversing the current direction. (a) Injection Barrier Modulation (IBM) and (b) Bulk Conductivity Switching (BCS).

\subsubsection{Bulk Conductivity Switching}

The conductivity switching in $\alpha$-CBT is still an interfacial effect and thereby sensitive to the quality of the interface between the active material and the electrode. In the quest to obtain true BCS, Paper [1] presents several semiconducting ferroelectrics that are designed still following the core-shell principle. Here we will focus on the SubPc-amide in Fig. 2.18b, as the synthesis of the Pc-oVDF and PBI-oVDF compounds has proven particularly difficult $[1,100]$.

Since it follows the same design as previous compounds, it should come to no surprise that also SubPc-Amide forms a columnar hexagonal liquid crystal [53]. This time however, the material shows proper bulk ferroelectric properties such as a saturated hysteresis loop. That, combined with the modest bandgap in the visible range of about $2.2 \mathrm{eV}$, makes SubPc-Amide one of the first true semiconducting ferroelectrics.

The current voltage curve again shows conductivity switching at the ferroelectric coercive field and upon reversing the current direction. However, the loop sense as shown in Fig. 4.3b is opposite to that of IBM and other conductivity switching mechanisms. This suggests that a completely different and unique mechanism is at play, namely a bulk conductivity switching (BCS). This is confirmed by the fact that both the on and off state curves can only be described as a sum of Ohmic and SCLC, both of which are bulk descriptions. The asymmetric potential caused by the aligned dipoles is essentially a ratchet and causes an asymmetry in the bulk charge transport. A more detailed explanation of this mechanism and the corresponding model will be presented in Section 4.3. 


\subsubsection{Tuning the Conductivity Switching towards Applications}

The occurrence of IBM and BCS in two different material and electrode combinations raises the question whether both mechanisms could occur in the same system. This question is resolved with the BTTTA material from Fig. 2.18c, where both IBM and BCS are observed, sometimes even both in the same device [8]. Which of the two competing mechanisms is dominant depends on the chosen electrodes, the temperature, and the sweep speed. At lower fields, IBM is expected to dominate because only partial switching of the polarization, which starts preferentially at the electrodes, is sufficient to modulate the injection barrier as in $\alpha$-CBT. BCS requires the polarization to be fully switched because even a few unswitched columns keep the conductivity high due to percolation effects.

While the loop sense is irrelevant for applications, it is a good indicator of the dominant conductivity switching mechanism. We would like to stress the uniqueness of the BCS mechanism. Most conductivity modulation mechanisms are based on an interface effect: from the SC/FE blends to a (ferroelectric) Schottky diode or a simple FET.

The advantage of having a single material over the blends is that there is no need to precisely control phase separation and the resulting morphology. Furthermore, having a bulk effect means that there is also no need to have high quality interfaces with the electrodes. The small size of a single column could allow bit densities up to 100 times higher than in the blends. In symmetric devices, crosstalk between neighboring cells in a crossbar memory could still be an issue. Rectification that prevents this crosstalk can be obtained with asymmetric electrodes, where one electrode is completely non-injecting, e.g. aluminum.

An issue with the devices based on $\alpha$-CBT and subPc-Amide is their poor retention time of only several minutes. This is fortunately not the case for BTTTA. As demonstrated by the kMC simulation in Fig. 3.9b, the coercive field of BTTTA is relatively high, which indicates a high barrier for polarization switching. This can be attributed to the precise molecular packing of the materials that does not allow the z-flip, which would be the most energetically favorable mode of dipole flipping as discussed in Section 3.3.3.2. Furthermore, BTTTA seems to be highly ordered, which as we know from the kMC simulations in Section 3.3.3 is crucial for good retention. These two mechanisms lead to near infinite retention times at room temperature, exceeding that of $\mathrm{P}(\mathrm{VDF}-\mathrm{TrFE})$, and also exceeding the commercial requirement of 10 years even at elevated temperatures.

\subsection{Asymmetric Hopping Model}

In the previous section we introduced the BCS mechanism that appears in semiconducting ferroelectrics with injecting electrodes. In this section we will 
have a closer look at this mechanism and develop a model that can quantitatively describe the experimental conductivity switching. Before we introduce this model however, we will first briefly discuss charge transport in organic semiconductors in general.

\subsubsection{Hopping Charge Transport}

Charge carriers in organic electronics are generally localized on molecular sites. Charge transport occurs through thermally activated tunneling between these sites, generally referred to as hopping [225]. The simplest way to describe this hopping from a site $i$ to a site $j$ is given by the Miller Abrahams hopping rate $[226]$ :

$$
\nu=\nu_{0} \exp \left(-2 \alpha R_{i j}\right) \begin{cases}\exp \left(-\Delta U_{i j} / k_{B} T\right), & \text { if } \Delta U_{i j}>0 \\ 1, & \text { otherwise }\end{cases}
$$

Note the similarity with Eq. (3.13): a hop upwards in energy is penalized with a Boltzmann factor. Here $\alpha$ is the inverse localization length, $R_{i j}$ the distance, and $\Delta U_{i j}$ the energy difference between the sites. The latter two parameters are not fixed but vary between sites due to the disordered nature of organic materials, and their distribution can often be approximated with a Gaussian.

A charge carrier localized on a site causes a slight distortion of its environment. This distortion together with the charge carrier is called a polaron, and polaronic effects can affect the charge transport properties. This is usually modeled with the Marcus theory for electron transfer [227]. This theory assumes that the polaronic distortion can be described with a parabolic potential that is characterized by the reorganization energy $\lambda$. The charge carrier then moves in this potential landscape through thermal activation over the energy barriers between molecules. This is schematically shown in Fig. 4.4a, where the figure on the right shows the effect of an applied field.

With an applied field the energy barrier between two molecules is given

by $\Delta U_{i j}=\frac{R_{i j}^{2}}{4 \lambda}\left(\lambda+\Delta U_{0} \pm E R_{i j}\right)^{2}$. The thermal activation over this barrier gives a hopping rate of $\nu=\nu_{0} \exp \left(\Delta U_{i j} / k_{B} T\right)$. Note that upon increasing the applied field, the energy barrier will eventually be zero and the maximal hopping rate $\nu_{0}$ is obtained. A further increase of the field counterintuitively leads to a decrease of the hopping rate. This is called the Marcus inverted regime, and is an effect unique to Marcus hopping.

\subsubsection{Asymmetric Charge Transport}

The hopping rates in the previous section describe a single hop between two molecules. To go from this microscopic description to a macroscopic current, one somehow needs to average over the hopping between all the disordered 


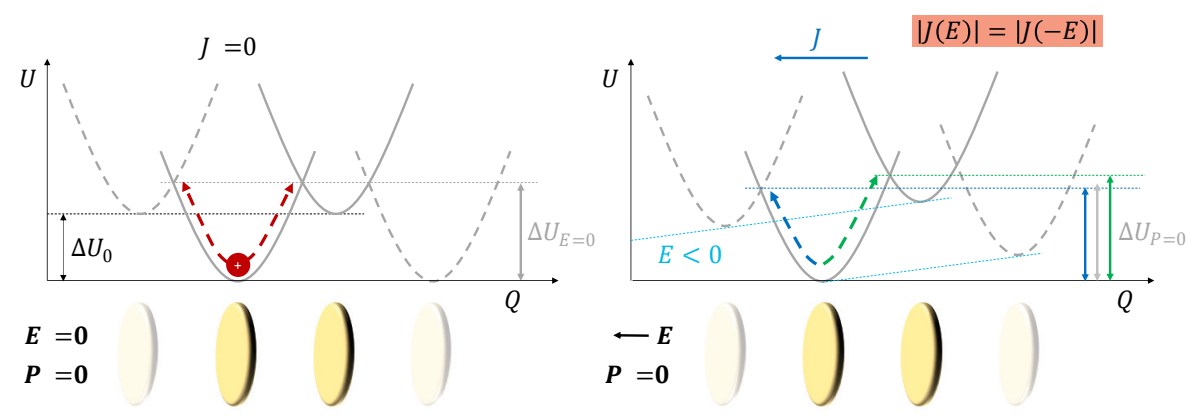

(a)

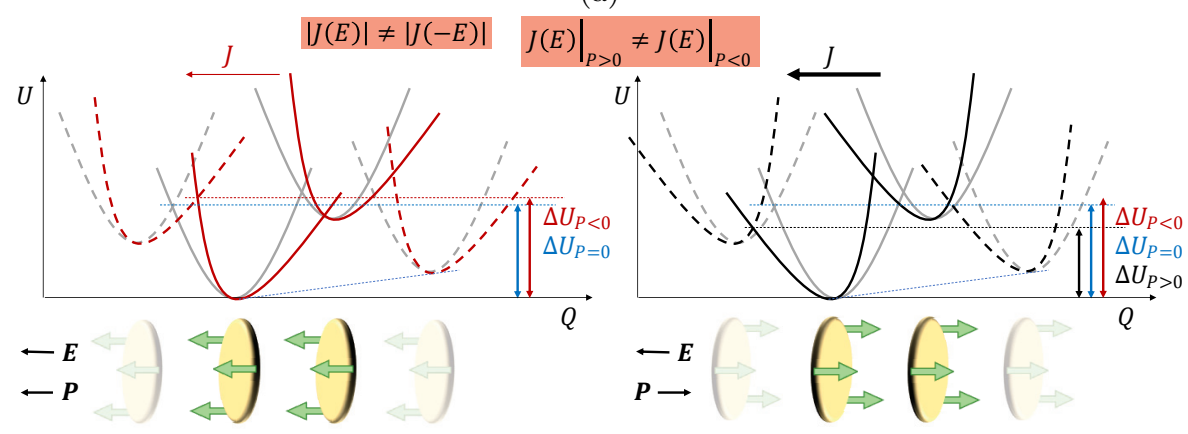

(b)

Figure 4.4: The hopping transport of a charge carrier (hole) through a potential landscape of Marcus parabolas. A simple two-site model with periodic boundaries is considered. (a) Symmetric hopping without (left) or with (right) applied field E. (b) Asymmetric hopping caused by the polarization pointing left or right.

sites in the system. While analytical approximations for this have been made [225], a more straightforward way is to use kMC modeling. This has become a powerful tool to analyze charge transport in a large variety of organic electronic devices due to its versatility [228].

In our case, an even simpler approach is sufficient to understand the asymmetric charge transport in semiconducting ferroelectrics. The charge transport in these materials is mostly one dimensional, with very little charge transport between neighboring columns due to the insulating tails separating them [95, 96]. Percolation effects therefore play an important role, and it is expected that the charge transport is determined by a few critical ratelimiting steps with a large energy barrier. It is therefore sufficient to focus on this critical hop with the two-site model illustrated in Fig. 4.4. While this model won't be able to predict absolute currents, it can predict the on/off ratio of the conductivity switching. 
The symmetric parabolas of Fig. 4.4a lead to a current that is symmetric in the applied field: $|J(E)|=|J(-E)|$. An asymmetry is then introduced through the asymmetric potential caused by the aligned dipoles, which is added to the Marcus parabolas. We can exactly calculate this potential using the morphology of the supramolecular column. The potential is evaluated at the area where the charge transport takes place within the core of the column, which is based on DFT calculations of the molecular orbitals of the material.

This leads to the situation in Fig. 4.4b. The asymmetry leads to a deor increase of the energy barrier for hopping $\Delta U$ depending on the relative direction of the polarization and the current. This gives an intuitive picture of how the asymmetric charge transport in Fig. $4.3 \mathrm{~b}$ is obtained. We can quantify the asymmetry by calculating the rate of all hops against and along the field, from which a mobility is obtained. The ratio of the mobility along and against the polarization direction can then be compared to the experimental on/off ratio.

The result of this calculation for SubPc-Amide is given in Fig. 4.5. We find a good agreement with the experimental on/off ratio at lower fields in Fig. 4.5a. Once the electric field approaches the coercive field, the polarization will start to switch and the experimental on/off ratio drops to 1 . The polarization reversal is not included in the simple model, and the calculated ratio therefore does not show this drop-off.

Surprisingly, Fig. 4.5b shows that the on/off ratio can become both larger and smaller than unity depending on the exact choice of parameters. This indicates that the effect of the asymmetric potential of the dipoles is not as straightforward as the intuitive picture in Fig. 4.4b suggests. It is a precise balance between the asymmetric dipole potential, the Marcus parabolas, and the applied field that determines how the asymmetry in the charge transport works out. Nevertheless, this simple model confirms that the experimentally observed asymmetry is a modulation of the bulk conductivity due to the symmetry breaking induced by the ferroelectric polarization. 


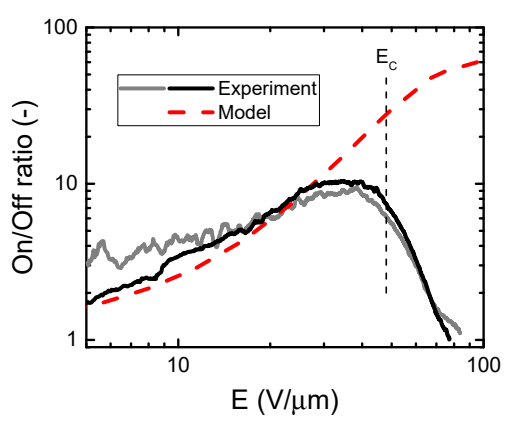

(a)

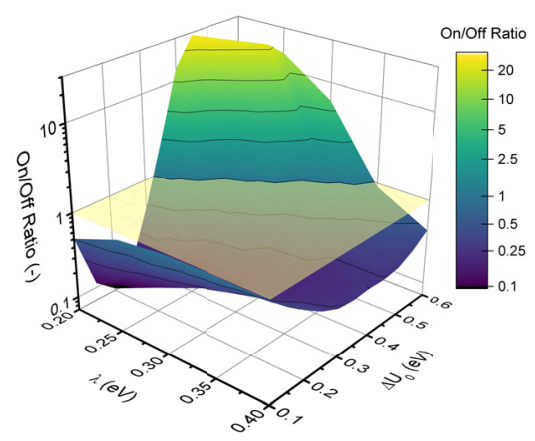

(b)

Figure 4.5: The on/off ratio for the charge transport in SubPc-Amide. (a) The ratio as a function of applied field, where typical parameters of $\lambda=0.2 \mathrm{eV}$ and $\Delta U_{0}=0.45 \mathrm{eV}$ were used. (b) The dependence of the ratio at $100 \mathrm{~V} / \mu \mathrm{m}$ on $\lambda$ and $\Delta U_{0}$. 


\section{Chapter 5}

\section{Conclusion and Outlook}

The aim of thesis has been to address the lack of understanding of organic ferroelectrics, so as to provide guidelines towards their improvement. For this, we have presented a variety of different models that are supported by experimental data. These models have been applied to a specific class of supramolecular discotic ferroelectrics, of which BTA is the prototype system. Nevertheless, most conclusions are valid for all discotic ferroelectrics or even all disordered ferroelectrics.

We have developed a microscopic electrostatic model that can simulate the flipping of the ferroelectric dipoles with a kMC approach. The simulated hysteresis loops and retention curves showed a good agreement with the experiments, demonstrating the validity of the model. The obtained parameter dependencies could be rationalized with the analytical theory of thermally activated nucleation limited switching.

The microscopic nature of the model provides a direct insight into the polarization switching processes. We found that nucleation happens at the contacts for field-driven switching (i.e. hysteresis loops), whereas during spontaneous depolarization it happens near defects that are induced by disorder. This means that if the disorder in the material is reduced, the retention time could be improved dramatically without greatly affecting the coercive field.

Further analysis of the nucleation in the simulations revealed a critical nucleation volume that decreases with applied field. This sparked the development of an analytical framework based on the theory of thermally activated nucleation limited switching. We were able to unify this theory with the phenomenological Merz law using the field-dependent nucleation volume. The resulting analytical model provides a coherent description of the switching transients in both simulations and experiments. It also gives a potential 
physical basis for the Merz law, which is often observed in experiments but ill understood.

This analytical description can be extended to include the dispersive switching that is observed in virtually all disordered ferroelectrics. This extension is based on the Preisach model, which considers a non-ideal ferroelectric as a collection of ideal hysterons. Through a series of experiments, we have directly related these hysterons and their distribution to the morphology of the material, thereby providing a physical basis for the originally mathematical Preisach model. This relation was verified with a simplified version of the electrostatic kMC model. The resulting model is particularly relevant when designing multilevel memories based on disordered ferroelectrics.

When looking towards applications, it is desirable to combine ferroelectricity with semiconductivity. This solves the problem of the destructive readout in memory applications, and opens the door towards new applications based on the interplay between the two properties. The supramolecular ferroelectrics in this thesis can be made semiconducting by increasing the size of the $\pi$-conjugated core.

These semiconducting ferroelectrics show two types of conductivity switching: injection barrier modulation (IBM) and bulk conductivity switching (BCS). IBM is an interface effect that has been observed before in semiconductor ferroelectric blends. BCS on the other hand is a unique effect that is observed with injecting electrodes. This BCS and the associated asymmetric conductivity can be understood as a result of the asymmetric potential landscape caused by the ferroelectric dipoles. Using a simple Marcus hopping model that incorporates this asymmetry, we can reproduce the experimental conductivity switching.

For a full understanding of the conductivity switching in these semiconducting ferroelectrics, an extension of the simple hopping model presented in this thesis would be necessary. This could be a kMC hopping model that takes into account the full morphology of the material as well as the device structure, instead of just considering the critical hop. Finally, this hopping model could be integrated with the $\mathrm{kMC}$ model for ferroelectric switching. This would give a full description of the behavior of the semiconducting ferroelectrics and might reveal further effects of the interplay between the switching dipoles and the hopping charges.

The semiconducting ferroelectrics can find a straightforward application as the non-volatile memory in organic electronic devices. An exciting extension of this application is the use of these materials for neuromorphic devices, which requires memristive elements with multilevel storage. The supramolecular semiconducting ferroelectrics fulfill this criterion, and our Preisach model can describe their performance in this regard.

Another interesting opportunity lies in the photovoltaic properties. The ferroelectric asymmetry in these materials could help separate photogenerated charge carriers and thereby make efficient solar cells. It is possible to get above 
band gap photovoltages according to the bulk photovoltaic effect, which could lead to photovoltaic applications where high voltages are required. Initial results on these materials indicate that there is a ferroelectrically switchable photovoltage/current, but this is the subject of ongoing work.

To conclude, this thesis presents a combination of models and experiments that lead to an increased understanding of ferroelectrics. From the fundamental physics side, this has led to some intriguing observations, such as the physical validity of the Preisach model and the occurrence of bulk conductivity switching in semiconducting ferroelectrics. From the application point of view, the insights gained point to key parameters such as disorder that govern the performance of ferroelectric devices. This provides guidelines for the design of organic ferroelectrics for future applications in organic electronics. 


\section{Acknowledgments}

You have made it through this thesis! Or more likely, you skipped to this section to see if you are mentioned, for which I do not blame you. In either case, let me start by thanking you, the reader, for showing interest in the work I've been doing for the past years.

The most important person to thank is of course my supervisor Martijn Kemerink, for guiding me through the journey of getting a $\mathrm{PhD}$. I am very grateful that you gave me the opportunity to do research in your group, even though I broke the same-nationality rule of the group by also being Dutch. Thank you for always guiding me in the right direction, but also leaving me free to explore things on my own. Your open door policy, and your availability in general, has always been much appreciated. Whenever we ran into problems, you never had a lack of ideas to solve them, and your contagious enthusiasm helped me stay motivated. I am thankful for your support in not only research matters, but also in professional development, such as your support for my participation in the Lindau meeting. I am sure that all these qualities will make your research group thrive in Heidelberg! Thank you also for enjoyable moments outside of work; it was especially nice to have someone who would not get bored of talking about (mountain)biking. It is too bad we did not manage to go on more rides together!

I am also grateful to my co-supervisor Mats Fahlman, who even though he was not directly involved, has always showed an interest in our work. I would like to thank my opponent, Kamal Asadi, and my defense committee members Katia Gallo, Kristian Berland, and Charlie Karis, for taking the time to read and evaluate my thesis. I am sure we will have interesting discussions during my $\mathrm{PhD}$ defense.

It has been great to have been part of the group of CoMaDe, whose members have now spread out over the world. Davood, Doro, Guangzheng, Hassan, 
Indre, Nikos, Olof, Sebastian, and Tanvi, thank you for providing such an enjoyable work environment. Our weekly fika was always a great way to break the week in half and sample treats from the different cultures represented in the group.

There is one CoMaDean that deserves her own section: my team- and officemate Indre. Indre, you allowed me to hit the ground running when I started, and without your help this thesis would not exist. Your experimental prowess is unparalleled, and you managed to get even the trickiest materials to obey. I think we made a great match with your experimental skills and my affinity for modeling. Not only professionally, but also personally you have been a pleasure to work with. I particularly enjoyed exploring new cities with you, while trying to sell our strategy of supramolecular ferroelectrics at conferences all over the world. Thank you also for your patience during my sometimes clumsy experimental efforts, and for enduring my rants against the computer whenever it was not cooperating.

No research group can function without administrative support. I thank Anna-Maria, Jenny, Emma, and Ann-Charlotte for their help over the years. I would also like to thank Jörgen for his support with more technical issues.

We would have had nothing to do experiments on if it weren't for all the organic chemists that we have had the pleasure of collaborating with in the groups of Rint Sijbesma, Miguel Garcia-Iglesias, and David GonzalezRodriguez. Their ability to make almost any molecule we could think of is astonishing and has been vital to our research. I am also grateful to Mathieu and Michal formerly from KTH for performing MD and DFT simulations that nicely supplemented our experimental and kMC work.

A very extended form of 'collaboration' has been with the Centre of Advanced Materials at Heidelberg University, which is the new home of Martijn's research group. In this context, I would like to thank Stefan, Olaf, Anton, Henna, and Martina, for all their help in setting up our lab in Heidelberg. I hope the research will thrive there!

Moving towards the more informal forms of support, I have to thank all my friends and colleagues at IFM. This list of people includes but is not limited to Andreas, Arnaud, Claudia, Clio, Davide, Hassan, Johan (who deserves a special thanks for the Swedish translation of the popular science summary), Judit, Laurent, Marius, Max, Mike, Nikos, Olof, Riccardo, Teja, and Victor. Both at and outside of work you provided the opportunity to discuss research, PhD struggles, politics, culture, living in Sweden, or just to hang out while enjoying drinks, food, and music.

I'm happy to have been part of the graduate school Agora Materiae, which has not only been a platform for a broader insight into materials science, but 
also a great way to make friends. I will especially remember the trip to Italy that we organized. I am particularly thankful to Per-Olof, Fredrik, Caroline, and Karina, for making Agora into the stimulating and enjoyable environment that it is.

I have been fortunate to have shared my apartment in Linköping with a variety of great roommates. In particular, I would like to thank Klein, who has shared the apartment with me for most of my $\mathrm{PhD}$, and Alex, for livening up the apartment and bringing Bacon with her. I hope that you, Davide, and Victor take good care of the apartment and particularly the garden!

That brings me to my friends at 'homehome', starting with the Studybuddies. It was always great seeing you again whenever we managed to arrange a date. But also digitally we've had a lot of fun, interesting, and helpful conversations. Hopefully we can see each other a bit more in the coming years! I'll express a similar wish for my outdoor/mountainbiking friends in Eindhoven; I hope that we can hang out and go for rides together more often now than just on New Year's Eve! Finally, I would also like to thank Jaimy and Pedram for our gaming sessions and tolerating my lack of Rocket League skills.

If it weren't for the travelling associated with this thesis work, I never would have met Erin. While we were separated by a long distance at first, we have been fortunate to live together during these unfortunate times. Your support during this final phase of my $\mathrm{PhD}$ has been invaluable, as well as your help in figuring out what to do next.

I would like to thank my family for their continuous support; my siblings and siblings in law for always being ready to help, my niece and nephew for never failing to brighten up my day, and my grandparents for always showing interest in my progress. Finally, I am very grateful to my parents, for always providing the opportunities I need to succeed in life. 


\section{Bibliography}

${ }^{1}$ A. V. Gorbunov, M. García-Iglesias, J. Guilleme, T. D. Cornelissen, W. S. C. Roelofs, T. Torres, D. González-Rodríguez, E. W. Meijer, and M. Kemerink, "Ferroelectric self-assembled molecular materials showing both rectifying and switchable conductivity," Science Advances 3, e1701017 (2017).

${ }^{2}$ I. Urbanavičiūtè, T. D. Cornelissen, X. Meng, R. P. Sijbesma, and M. Kemerink, "Physical reality of the Preisach model for organic ferroelectrics," Nature Communications 9, 4409 (2018).

${ }^{3}$ T. D. Cornelissen, M. Biler, I. Urbanaviciute, P. Norman, M. Linares, and M. Kemerink, "Kinetic Monte Carlo simulations of organic ferroelectrics," Physical Chemistry Chemical Physics 21, 1375-1383 (2019).

${ }^{4}$ T. D. Cornelissen, I. Urbanaviciute, and M. Kemerink, "Microscopic model for switching kinetics in organic ferroelectrics following the Merz law," Physical Review B 101, 214301 (2020).

${ }^{5}$ I. Urbanavičiūtè, X. Meng, T. D. Cornelissen, A. V. Gorbunov, S. Bhattacharjee, R. P. Sijbesma, and M. Kemerink, "Tuning the Ferroelectric Properties of Trialkylbenzene-1,3,5-tricarboxamide (BTA)," Advanced Electronic Materials, 1600530 (2017).

${ }^{6}$ I. Urbanavičiūtè, S. Bhattacharjee, M. Biler, J. A. M. Lugger, T. D. Cornelissen, P. Norman, M. Linares, R. P. Sijbesma, M. Kemerink, I. Urbanaviciute, S. Bhattacharjee, M. Biler, J. A. M. Lugger, T. D. Cornelissen, P. Norman, M. Linares, R. P. Sijbesma, and M. Kemerink, "Suppressing depolarization by tail substitution in an organic supramolecular ferroelectric," Physical Chemistry Chemical Physics 21, 2069-2079 (2019). 
${ }^{7}$ I. Urbanavičiūtè, X. Meng, M. Biler, Y. Wei, T. D. Cornelissen, S. Bhattacharjee, M. Linares, and M. Kemerink, "Negative piezoelectric effect in an organic supramolecular ferroelectric," Materials Horizons 6, 1688-1698 (2019).

${ }^{8}$ N. M. Casellas, I. Urbanaviciute, T. D. Cornelissen, J. A. Berrocal, T. Torres, M. Kemerink, and M. García-Iglesias, "Resistive switching in an organic supramolecular semiconducting ferroelectric," Chemical Communications 55, 8828-8831 (2019).

${ }^{9}$ E. Cantatore, ed., Applications of Organic and Printed Electronics, Integrated Circuits and Systems (Springer US, Boston, MA, 2013).

${ }^{10}$ V. M. Fridkin, Photoferroelectrics, Vol. 9, Springer Series in Solid-State Sciences (Springer Berlin Heidelberg, Berlin, Heidelberg, 1979).

${ }^{11}$ M. Fiebig, T. Lottermoser, D. Meier, and M. Trassin, "The evolution of multiferroics," Nature Reviews Materials 1, 16046 (2016).

${ }^{12}$ K. Asadi, D. M. de Leeuw, B. de Boer, and P. W. M. Blom, "Organic non-volatile memories from ferroelectric phase-separated blends," Nature Materials 7, 547-550 (2008).

${ }^{13}$ Xerox, Xerox Digital Labels, https ://www.xerox.com/en-us/insights/ digital-labels (visited on 03/23/2020).

${ }^{14}$ G. M. Sessler, ed., Electrets, Vol. 33, Topics in Applied Physics (Springer Berlin Heidelberg, Berlin, Heidelberg, 1987).

${ }^{15}$ T. Correia and Q. Zhang, eds., Electrocaloric Materials, Vol. 34, Engineering Materials (Springer Berlin Heidelberg, Berlin, Heidelberg, 2014), pp. 147-182.

${ }^{16}$ G. Heckmann, "Die Gittertheorie der festen Körper," in Ergebnisse der exakten naturwissenschaften (Springer Berlin Heidelberg, Berlin, Heidelberg), pp. 100-153.

${ }^{17}$ S. T. Lagerwall, Ferroelectric and Antiferroelectric Liquid Crystals (Wiley, Weinheim, Germany, 1999).

${ }^{18}$ C. Kittel, "Theory of Antiferroelectric Crystals," Physical Review 82, 729732 (1951).

${ }^{19}$ C. F. Pulvari, "Ferrielectricity," Physical Review 120, 1670-1673 (1960).

${ }^{20}$ L. Jin, F. Li, and S. Zhang, "Decoding the Fingerprint of Ferroelectric Loops: Comprehension of the Material Properties and Structures," Journal of the American Ceramic Society 97, 1-27 (2014).

${ }^{21}$ T. Schenk, E. Yurchuk, S. Mueller, U. Schroeder, S. Starschich, U. Böttger, and T. Mikolajick, "About the deformation of ferroelectric hystereses," Applied Physics Reviews 1, 041103 (2014). 
${ }^{22}$ Z. Zhou, Q. Yang, M. Liu, Z. Zhang, X. Zhang, D. Sun, T. Nan, N. Sun, and X. Chen, "Antiferroelectric Materials, Applications and Recent Progress on Multiferroic Heterostructures," SPIN 05, 1530001 (2015).

${ }^{23}$ K. Zou, Y. Dan, H. Xu, Q. Zhang, Y. Lu, H. Huang, and Y. He, "Recent advances in lead-free dielectric materials for energy storage," Materials Research Bulletin 113, 190-201 (2019).

${ }^{24}$ M. Guo, M. Wu, W. Gao, B. Sun, and X. Lou, "Giant negative electrocaloric effect in antiferroelectric $\mathrm{PbZrO} 3$ thin films in an ultra-low temperature range," Journal of Materials Chemistry C 7, 617-621 (2019).

${ }^{25}$ R. D. King-Smith and D. Vanderbilt, "Theory of polarization of crystalline solids," Physical Review B 47, 1651-1654 (1993).

${ }^{26}$ R. Resta, "Macroscopic Electric Polarization as a Geometric Quantum Phase," Europhysics Letters (EPL) 22, 133-138 (1993).

${ }^{27}$ N. A. Spaldin, "A beginner's guide to the modern theory of polarization," Journal of Solid State Chemistry 195, 2-10 (2012).

${ }^{28}$ A. Devonshire, "XCVI. Theory of barium titanate," The London, Edinburgh, and Dublin Philosophical Magazine and Journal of Science 40, 1040-1063 (1949).

${ }^{29}$ P. Chandra and P. B. Littlewood, "A Landau Primer for Ferroelectrics," in Physics of ferroelectrics, Vol. 105 (Springer Berlin Heidelberg, Berlin, Heidelberg, 2007), pp. 69-116.

${ }^{30}$ M. E. Lines and A. M. Glass, Principles and Applications of Ferroelectrics and Related Materials (Oxford University Press, 1997).

${ }^{31}$ V. M. Fridkin and S. Ducharme, "General features of the intrinsic ferroelectric coercive field," Physics of the Solid State 43, 1320-1324 (2001).

${ }^{32}$ J. F. Scott, "Switching of Ferroelectrics Without Domains," Advanced Materials 22, 5315-5317 (2010).

${ }^{33}$ J. F. Scott, "A review of ferroelectric switching," Ferroelectrics 503, 117132 (2016).

${ }^{34}$ C. B. Sawyer and C. H. Tower, "Rochelle Salt as a Dielectric," Physical Review 35, 269-273 (1930).

${ }^{35}$ M. Fukunaga and Y. Noda, "New technique for measuring ferroelectric and antiferroelectric hysteresis loops," Journal of the Physical Society of Japan 77, 1-5 (2008).

${ }^{36}$ J. F. Scott, "Ferroelectrics go bananas," Journal of Physics: Condensed Matter 20, 021001 (2007).

${ }^{37}$ H. Ishii, T. Nakajima, Y. Takahashi, and T. Furukawa, "Ultrafast Polarization Switching in Ferroelectric Polymer Thin Films at Extremely High Electric Fields," Applied Physics Express 4, 031501 (2011). 
${ }^{38}$ A. K. Tagantsev, I. Stolichnov, N. Setter, J. S. Cross, and M. Tsukada, "Non-Kolmogorov-Avrami switching kinetics in ferroelectric thin films," Physical Review B 66, 214109 (2002).

${ }^{39}$ R. R. Mehta, B. D. Silverman, and J. T. Jacobs, "Depolarization fields in thin ferroelectric films," Journal of Applied Physics 44, 3379 (1973).

${ }^{40}$ X. J. Lou, "Polarization retention on short, intermediate, and long time scales in ferroelectric thin films," Journal of Applied Physics 105, 094107 (2009).

${ }^{41}$ A. V. Gorbunov, X. Meng, I. Urbanaviciute, T. Putzeys, M. Wübbenhorst, R. P. Sijbesma, and M. Kemerink, "Polarization loss in the organic ferroelectric trialkylbenzene-1,3,5-tricarboxamide (BTA)," Physical Chemistry Chemical Physics 19, 3192-3200 (2017).

${ }^{42}$ A. K. Tagantsev, L. E. Cross, and J. Fousek, Domains in Ferroic Crystals and Thin Films (Springer New York, New York, NY, 2010).

${ }^{43}$ A. Pramanick, A. D. Prewitt, J. S. Forrester, and J. L. Jones, "Domains, Domain Walls and Defects in Perovskite Ferroelectric Oxides: A Review of Present Understanding and Recent Contributions," Critical Reviews in Solid State and Materials Sciences 37, 243-275 (2012).

${ }^{44}$ J. Seidel, L. W. Martin, Q. He, Q. Zhan, Y.-H. Chu, A. Rother, M. E. Hawkridge, P. Maksymovych, P. Yu, M. Gajek, N. Balke, S. V. Kalinin, S. Gemming, F. Wang, G. Catalan, J. F. Scott, N. A. Spaldin, J. Orenstein, and R. Ramesh, "Conduction at domain walls in oxide multiferroics," Nature Materials 8, 229-234 (2009).

${ }^{45}$ G. Catalan, J. Seidel, R. Ramesh, and J. F. Scott, "Domain wall nanoelectronics," Reviews of Modern Physics 84, 119-156 (2012).

${ }^{46}$ E. Soergel, "Piezoresponse force microscopy (PFM)," Journal of Physics D: Applied Physics 44, 464003 (2011).

${ }^{47}$ A. Gruverman and A. Kholkin, "Nanoscale ferroelectrics: processing, characterization and future trends," Reports on Progress in Physics 69, 24432474 (2006).

${ }^{48}$ N. Balke, P. Maksymovych, S. Jesse, A. Herklotz, A. Tselev, C. B. Eom, I. I. Kravchenko, P. Yu, and S. V. Kalinin, "Differentiating Ferroelectric and Nonferroelectric Electromechanical Effects with Scanning Probe Microscopy," ACS Nano 9, 6484-6492 (2015).

${ }^{49}$ S. M. Yang, A. N. Morozovska, R. Kumar, E. A. Eliseev, Y. Cao, L. Mazet, N. Balke, S. Jesse, R. K. Vasudevan, C. Dubourdieu, and S. V. Kalinin, "Mixed electrochemical-ferroelectric states in nanoscale ferroelectrics," Nature Physics 13, 812-818 (2017).

${ }^{50}$ J. Liu, W. Chen, B. Wang, and Y. Zheng, "Theoretical Methods of Domain Structures in Ultrathin Ferroelectric Films: A Review," Materials 7, 65026568 (2014). 
${ }^{51}$ A. V. Gorbunov, T. Putzeys, I. Urbanaviciute, R. A. J. Janssen, M. Wübbenhorst, R. P. Sijbesma, and M. Kemerink, "True ferroelectric switching in thin films of trialkylbenzene-1,3,5-tricarboxamide (BTA)," Physical Chemistry Chemical Physics 18, 23663-23672 (2016).

${ }^{52}$ H. Takezoe, K. Kishikawa, and E. Gorecka, "Switchable columnar phases," Journal of Materials Chemistry 16, 2412-2416 (2006).

${ }^{53}$ J. Guilleme, E. Cavero, T. Sierra, J. Ortega, C. L. Folcia, J. Etxebarria, T. Torres, and D. González-Rodríguez, "Polar Switching in a Lyotropic Columnar Nematic Liquid Crystal Made of Bowl-Shaped Molecules," Advanced Materials 27, 4280-4284 (2015).

${ }^{54}$ S. B. Lang and D. K. Das-Gupta, "Laser-intensity-modulation method: A technique for determination of spatial distributions of polarization and space charge in polymer electrets," Journal of Applied Physics 59, 21512160 (1986).

${ }^{55}$ J. Valasek, "Piezo-Electric and Allied Phenomena in Rochelle Salt," Physical Review 17, 475-481 (1921).

${ }^{56}$ N. Setter, D. Damjanovic, L. Eng, G. Fox, S. Gevorgian, S. Hong, A. I. Kingon, H. Kohlstedt, N. Y. Park, G. B. Stephenson, I. Stolichnov, A. K. Tagantsev, D. V. Taylor, T. Yamada, and S. Streiffer, "Ferroelectric thin films: Review of materials, properties, and applications," Journal of Applied Physics 100, 051606 (2006).

${ }^{57}$ Y. Wang, W. Chen, B. Wang, and Y. Zheng, "Ultrathin Ferroelectric Films: Growth, Characterization, Physics and Applications," Materials 7, 63776485 (2014).

${ }^{58}$ L. W. Martin and A. M. Rappe, "Thin-film ferroelectric materials and their applications," Nature Reviews Materials 2, 16087 (2017).

${ }^{59}$ I. Urbanaviciute, "Multifunctional Supramolecular Organic Ferroelectrics," PhD thesis (Linköping, 2019).

${ }^{60}$ S. Horiuchi and Y. Tokura, "Organic ferroelectrics," Nature Materials 7, 357-366 (2008).

${ }^{61}$ R. Whatmore, "Ferroelectric Materials," in Springer handbook of electronic and photonic materials, edited by S. Kasap and P. Capper (Springer International Publishing, Especi, 2017), pp. 589-614.

${ }^{62}$ J. Rödel, K. G. Webber, R. Dittmer, W. Jo, M. Kimura, and D. Damjanovic, "Transferring lead-free piezoelectric ceramics into application," Journal of the European Ceramic Society 35, 1659-1681 (2015).

${ }^{63}$ S. Zhukov, H. Kungl, Y. A. Genenko, and H. von Seggern, "Statistical electric field and switching time distributions in PZT 1Nb2Sr ceramics: Crystal- and microstructure effects," Journal of Applied Physics 115, 014103 (2014). 
${ }^{64}$ Y. A. Genenko, S. Zhukov, S. Yampolskii, J. Schütrumpf, R. Dittmer, W. Jo, H. Kungl, M. Hoffmann, and H. von Seggern, "Universal Polarization Switching Behavior of Disordered Ferroelectrics," Advanced Functional Materials 22, 2058-2066 (2012).

${ }^{65}$ N. S. Dalal and A. Bussmann-Holder, eds., Ferro- and Antiferroelectricity: Order/Disorder versus Displacive, Vol. 124, Structure and Bonding (Springer Berlin Heidelberg, Berlin, Heidelberg, 2007).

${ }^{66}$ A. L. Solomon, "Thiourea, a New Ferroelectric," Physical Review 104, 1191-1191 (1956).

${ }^{67}$ A. S. Tayi, A. Kaeser, M. Matsumoto, T. Aida, and S. I. Stupp, "Supramolecular ferroelectrics.," Nature chemistry 7, 281-294 (2015).

${ }^{68}$ T. Furukawa, M. Date, and E. Fukada, "Hysteresis phenomena in polyvinylidene fluoride under high electric field," Journal of Applied Physics 51, 1135-1141 (1980).

${ }^{69}$ T. Furukawa and G. E. Johnson, "Measurements of ferroelectric switching characteristics in polyvinylidene fluoride," Applied Physics Letters 38, 1027-1029 (1981).

${ }^{70}$ H. S. Nalwa, ed., Ferroelectric polymers : chemistry, physics, and applications (M. Dekker Inc, 1995).

${ }^{71}$ I. Katsouras, K. Asadi, M. Li, T. B. van Driel, K. S. Kjær, D. Zhao, T. Lenz, Y. Gu, P. W. M. Blom, D. Damjanovic, M. M. Nielsen, and D. M. de Leeuw, "The negative piezoelectric effect of the ferroelectric polymer poly(vinylidene fluoride)," Nature Materials 15, 78-84 (2015).

${ }^{72}$ X. Chen, X. Han, and Q.-D. Shen, "PVDF-Based Ferroelectric Polymers in Modern Flexible Electronics," Advanced Electronic Materials 3, 1600460 (2017).

${ }^{73}$ B. Stadlober, M. Zirkl, and M. Irimia-Vladu, "Route towards sustainable smart sensors: ferroelectric polyvinylidene fluoride-based materials and their integration in flexible electronics," Chemical Society Reviews 48, 1787-1825 (2019).

${ }^{74}$ K. Asadi, M. Li, P. W. M. Blom, M. Kemerink, and D. M. de Leeuw, "Organic ferroelectric opto-electronic memories," Materials Today 14, 592599 (2011).

${ }^{75}$ H. Li, R. Wang, S. Han, and Y. Zhou, "Ferroelectric polymers for non-volatile memory devices: a review," Polymer International, pi.5980 (2020).

${ }^{76}$ P.-P. Shi, Y.-Y. Tang, P.-F. Li, W.-Q. Liao, Z.-X. Wang, Q. Ye, and R.-G. Xiong, "Symmetry breaking in molecular ferroelectrics," Chemical Society Reviews 45, 3811-3827 (2016). 
${ }^{77}$ S. Horiuchi, Y. Tokunaga, G. Giovannetti, S. Picozzi, H. Itoh, R. Shimano, R. Kumai, and Y. Tokura, "Above-room-temperature ferroelectricity in a single-component molecular crystal.," Nature 463, 789-792 (2010).

${ }^{78}$ S. Horiuchi, K. Kobayashi, R. Kumai, and S. Ishibashi, "Proton tautomerism for strong polarization switching," Nature Communications 8, 14426 (2017).

${ }^{79}$ A. S. Tayi, A. Shveyd, A. C.-H. Sue, J. M. Szarko, B. S. Rolczynski, D. Cao, T. J. Kennedy, A. A. Sarjeant, C. L. Stern, W. F. Paxton, W. Wu, S. K. Dey, A. C. Fahrenbach, J. R. Guest, H. Mohseni, L. X. Chen, K. L. Wang, J. F. Stoddart, and S. I. Stupp, "Room-temperature ferroelectricity in supramolecular networks of charge-transfer complexes," Nature 488, 485-489 (2012).

${ }^{80}$ R. Meyer, L. Liebert, L. Strzelecki, and P. Keller, "Ferroelectric liquid crystals," Journal de Physique Lettres 36, 69-71 (1975).

${ }^{81}$ H. Takezoe, "Polar liquid crystals - ferro, antiferro, banana, and columnar -," Molecular Crystals and Liquid Crystals 646, 46-65 (2017).

${ }^{82}$ H. Takezoe, "Historical Overview of Polar Liquid Crystals," Ferroelectrics 468, 1-17 (2014).

${ }^{83}$ D. Miyajima, F. Araoka, H. Takezoe, J. Kim, K. Kato, M. Takata, and T. Aida, "Ferroelectric Columnar Liquid Crystal Featuring Confined Polar Groups Within Core-Shell Architecture," Science 336, 209-213 (2012).

${ }^{84}$ H. Takezoe and F. Araoka, "Polar columnar liquid crystals," Liquid Crystals 41, 393-401 (2014).

${ }^{85}$ M. L. Nguyen and B.-K. Cho, "Ferroelectrically Switchable Axial Polarization in Columnar Liquid Crystalline Phases," Chemistry - A European Journal, chem.201904884 (2020).

${ }^{86}$ P. J. M. Stals, J. C. Everts, R. de Bruijn, I. A. W. Filot, M. M. J. Smulders, R. Martín-Rapún, E. A. Pidko, T. F. A. de Greef, A. R. A. Palmans, and E. W. Meijer, "Dynamic Supramolecular Polymers Based on Benzene1,3,5-tricarboxamides: The Influence of Amide Connectivity on Aggregate Stability and Amplification of Chirality," Chemistry - A European Journal 16, 810-821 (2010).

${ }^{87}$ K. K. Bejagam, G. Fiorin, M. L. Klein, and S. Balasubramanian, "Supramolecular polymerization of benzene-1,3,5-tricarboxamide: A molecular dynamics simulation study," Journal of Physical Chemistry B 118, 5218-5228 (2014).

${ }^{88}$ S. Cantekin, T. F. A. de Greef, and A. R. A. Palmans, "Benzene-1,3,5tricarboxamide: a versatile ordering moiety for supramolecular chemistry.," Chemical Society reviews 41, 6125-37 (2012). 
${ }^{89}$ C. Kulkarni, E. W. Meijer, and A. R. A. Palmans, "Cooperativity Scale: A Structure-Mechanism Correlation in the Self-Assembly of Benzene-1,3,5tricarboxamides," Accounts of Chemical Research 50, 1928-1936 (2017).

${ }^{90}$ L. Brunsveld, A. Schenning, M. Broeren, H. Janssen, J. Vekemans, and E. Meijer, "Chiral Amplification in Columns of Self-Assembled N,N ,N -Tris(( S )-3,7-dimethyloctyl)benzene-1,3,5-tricarboxamide in Dilute Solution," Chemistry Letters 29, 292-293 (2000).

${ }^{91}$ C. F. C. Fitié, W. S. C. Roelofs, M. Kemerink, and R. P. Sijbesma, "Remnant polarization in thin films from a columnar liquid crystal," Journal of the American Chemical Society 132, 6892-6893 (2010).

${ }^{92}$ C. F. C. Fitié, W. S. C. Roelofs, P. C. M. M. Magusin, M. Wübbenhorst, M. Kemerink, and R. P. Sijbesma, "Polar Switching in Trialkylbenzene1,3,5-tricarboxamides," Journal of Physical Chemistry B 116, 3928-3937 (2012).

${ }^{93}$ A. Timme, R. Kress, R. Q. Albuquerque, and H. Schmidt, "Phase Behavior and Mesophase Structures of 1,3,5-Benzene- and 1,3,5Cyclohexanetricarboxamides: Towards an Understanding of the Losing Order at the Transition into the Isotropic Phase," Chemistry - A European Journal 18, 8329-8339 (2012).

${ }^{94}$ R. Q. Albuquerque, A. Timme, R. Kress, J. Senker, and H. Schmidt, "Theoretical Investigation of Macrodipoles in Supramolecular Columnar Stackings," Chemistry - A European Journal 19, 1647-1657 (2013).

${ }^{95}$ S. Sergeyev, W. Pisula, and Y. H. Geerts, "Discotic liquid crystals: a new generation of organic semiconductors," Chemical Society Reviews 36, 1902 (2007).

${ }^{96}$ T. Wöhrle, I. Wurzbach, J. Kirres, A. Kostidou, N. Kapernaum, J. Litterscheidt, J. C. Haenle, P. Staffeld, A. Baro, F. Giesselmann, and S. Laschat, "Discotic Liquid Crystals," Chemical Reviews 116, 1139-1241 (2016).

${ }^{97}$ T. Kato, M. Yoshio, T. Ichikawa, B. Soberats, H. Ohno, and M. Funahashi, "Transport of ions and electrons in nanostructured liquid crystals," Nature Reviews Materials 2, 17001 (2017).

${ }^{98}$ S. Laschat, A. Baro, N. Steinke, F. Giesselmann, C. Hägele, G. Scalia, R. Judele, E. Kapatsina, S. Sauer, A. Schreivogel, and M. Tosoni, "Discotic Liquid Crystals: From Tailor-Made Synthesis to Plastic Electronics," Angewandte Chemie - International Edition 46, 4832-4887 (2007).

${ }^{99}$ J. Guilleme, M. J. Mayoral, J. Calbo, J. Aragó, P. M. Viruela, E. Ortí, T. Torres, and D. González-Rodríguez, "Non-Centrosymmetric Homochiral Supramolecular Polymers of Tetrahedral Subphthalocyanine Molecules," Angewandte Chemie - International Edition 54, 2543-2547 (2015). 
${ }^{100}$ M. García-Iglesias, B. F. M. de Waal, A. V. Gorbunov, A. R. A. Palmans, M. Kemerink, and E. W. Meijer, "A Versatile Method for the Preparation of Ferroelectric Supramolecular Materials via Radical End-Functionalization of Vinylidene Fluoride Oligomers," Journal of the American Chemical Society 138, 6217-6223 (2016).

${ }^{101}$ A. V. Gorbunov, A. T. Haedler, T. Putzeys, R. H. Zha, H. Schmidt, M. Kivala, I. Urbanaviciute, M. Wübbenhorst, E. W. Meijer, and M. Kemerink, "Switchable Charge Injection Barrier in an Organic Supramolecular Semiconductor," ACS Applied Materials \& Interfaces 8, acsami.6b02988 (2016).

${ }^{102}$ M. Dawber, K. M. Rabe, and J. F. Scott, "Physics of thin-film ferroelectric oxides," Reviews of Modern Physics 77, 1083-1130 (2005).

${ }^{103}$ A. N. Kolmogorov, "On the Statistical Theory of Crystallization of Metals," Izv. Akad. Nauk SSSR, Ser. Mat. 3, 355-359 (1937).

${ }^{104}$ M. Avrami, "Kinetics of Phase Change. II Transformation-Time Relations for Random Distribution of Nuclei," The Journal of Chemical Physics 8, $212-224$ (1940).

${ }^{105}$ Y. Ishibashi and Y. Takagi, "Note on Ferroelectric Domain Switching," Journal of the Physical Society of Japan 31, 506-510 (1971).

${ }^{106}$ S. Hashimoto, H. Orihara, and Y. Ishibashi, "Study on D - E Hysteresis Loop of TGS Based on the Avrami-Type Model," Journal of the Physical Society of Japan 63, 1601-1610 (1994).

${ }^{107}$ H. Orihara, S. Hashimoto, and Y. Ishibashi, "A Theory of D-E Hysteresis Loop Based on the Avrami Model," Journal of the Physical Society of Japan 63, 1031-1035 (1994).

${ }^{108}$ V. Shur, E. Rumyantsev, and S. Makarov, "Kinetics of phase transformations in real finite systems: Application to switching in ferroelectrics," Journal of Applied Physics 84, 445-451 (1998).

${ }^{109}$ Y. W. So, D. J. Kim, T. W. Noh, J.-G. Yoon, and T. K. Song, "Polarization switching kinetics of epitaxial $\mathrm{Pb}(\mathrm{Zr0}$.4Ti0.6)O3 thin films," Applied Physics Letters 86, 092905 (2005).

${ }^{110}$ O. Lohse, M. Grossmann, U. Boettger, D. Bolten, and R. Waser, "Relaxation mechanism of ferroelectric switching in $\mathrm{Pb}(\mathrm{Zr}, \mathrm{Ti}) \mathrm{O} 3$ thin films," Journal of Applied Physics 89, 2332-2336 (2001).

${ }^{111}$ J. Y. Jo, H. S. Han, J. G. Yoon, T. K. Song, S.-H. Kim, and T. W. Noh, "Domain Switching Kinetics in Disordered Ferroelectric Thin Films," Physical Review Letters 99, 267602 (2007).

${ }^{112}$ W. J. Merz, "Domain Formation and Domain Wall Motions in Ferroelectric BaTiO3 Single Crystals," Physical Review 95, 690-698 (1954). 
${ }^{113}$ S. Zhukov, Y. A. Genenko, and H. von Seggern, "Experimental and theoretical investigation on polarization reversal in unfatigued lead-zirconatetitanate ceramic," Journal of Applied Physics 108, 014106 (2010).

${ }^{114}$ A. Nautiyal, K. C. Sekhar, N. P. Pathak, N. Dabra, J. S. Hundal, and R. Nath, "Polarization switching properties of spray deposited CsNO3: PVA composite films," Applied Physics A 99, 941-946 (2010).

${ }^{115}$ W. J. Hu, D.-M. Juo, L. You, J. Wang, Y.-C. Chen, Y.-H. Chu, and T. $\mathrm{Wu}$, "Universal Ferroelectric Switching Dynamics of Vinylidene Fluoridetrifluoroethylene Copolymer Films," Scientific Reports 4, 4772 (2015).

${ }^{116}$ D. Zhao, I. Katsouras, K. Asadi, P. W. M. Blom, and D. M. de Leeuw, "Switching dynamics in ferroelectric P(VDF-TrFE) thin films," Physical Review B 92, 214115 (2015).

${ }^{117}$ A.-Q. Jiang, H. J. Lee, C. S. Hwang, and T.-A. Tang, "Resolving the Landauer paradox in ferroelectric switching by high-field charge injection," Physical Review B 80, 024119 (2009).

${ }^{118}$ Q. Meng, M.-G. Han, J. Tao, G. Xu, D. O. Welch, and Y. Zhu, "Velocity of domain-wall motion during polarization reversal in ferroelectric thin films: Beyond Merz's Law," Physical Review B 91, 054104 (2015).

${ }^{119}$ H. Ishii, T. Nakajima, T. Furukawa, and S. Okamura, "Polarization Switching Dynamics of Vinylidene Fluoride/Trifluoroethylene Copolymer Thin Films under High Electric Field at Various Temperatures," Japanese Journal of Applied Physics 52, 041603 (2013).

${ }^{120}$ E. Fatuzzo, "Theoretical Considerations on the Switching Transient in Ferroelectrics," Physical Review 127, 1999-2005 (1962).

${ }^{121}$ M. Hayashi, "Kinetics of Domain Wall Motion in Ferroelectric Switching. I. General Formulation," Journal of the Physical Society of Japan 33, 616628 (1972).

${ }^{122}$ Y.-H. Shin, I. Grinberg, I.-W. Chen, and A. M. Rappe, "Nucleation and growth mechanism of ferroelectric domain-wall motion," Nature 449, 881884 (2007).

${ }^{123}$ S. Liu, I. Grinberg, and A. M. Rappe, "Intrinsic ferroelectric switching from first principles," Nature 534, 360-363 (2016).

${ }^{124}$ S. M. Yang, J. Y. Jo, T. H. Kim, J.-G. Yoon, T. K. Song, H. N. Lee, Z. Marton, S. Park, Y. Jo, and T. W. Noh, "Ac dynamics of ferroelectric domains from an investigation of the frequency dependence of hysteresis loops," Physical Review B 82, 174125 (2010).

${ }^{125}$ T. Tybell, P. Paruch, T. Giamarchi, and J.-M. Triscone, "Domain Wall Creep in Epitaxial Ferroelectric Pb(Zr0.2Ti0.8)O3 Thin Films," Physical Review Letters 89, 097601 (2002). 
${ }^{126}$ P. Chauve, T. Giamarchi, and P. Le Doussal, "Creep and depinning in disordered media," Physical Review B 62, 6241-6267 (2000).

${ }^{127}$ R. C. Miller and G. Weinreich, "Mechanism for the Sidewise Motion of $180^{\circ}$ Domain Walls in Barium Titanate," Physical Review 117, 1460-1466 (1960).

${ }^{128}$ J. Y. Jo, D. J. Kim, Y. S. Kim, S.-B. Choe, T. K. Song, J.-G. Yoon, and T. W. Noh, "Polarization Switching Dynamics Governed by the Thermodynamic Nucleation Process in Ultrathin Ferroelectric Films," Physical Review Letters 97, 247602 (2006).

${ }^{129}$ K. B. Chong, F. Guiu, and M. J. Reece, "Thermal activation of ferroelectric switching," Journal of Applied Physics 103, 014101 (2008).

${ }^{130}$ D. Zhao, T. Lenz, G. H. Gelinck, P. Groen, D. Damjanovic, D. M. de Leeuw, and I. Katsouras, "Depolarization of multidomain ferroelectric materials," Nature Communications 10, 2547 (2019).

${ }^{131}$ M. Vopsaroiu, J. Blackburn, M. G. Cain, and P. M. Weaver, "Thermally activated switching kinetics in second-order phase transition ferroelectrics," Physical Review B 82, 024109 (2010).

${ }^{132}$ M. Vopsaroiu, P. M. Weaver, M. G. Cain, M. J. Reece, and Kok Boon C., "Polarization dynamics and non-equilibrium switching processes in ferroelectrics," IEEE Transactions on Ultrasonics, Ferroelectrics and Frequency Control 58, 1867-1873 (2011).

${ }^{133}$ S. Zhukov, Y. A. Genenko, O. Hirsch, J. Glaum, T. Granzow, and H. von Seggern, "Dynamics of polarization reversal in virgin and fatigued ferroelectric ceramics by inhomogeneous field mechanism," Physical Review B 82, 014109 (2010).

${ }^{134}$ J. Schütrumpf, S. Zhukov, Y. A. Genenko, and H. von Seggern, "Polarization switching dynamics by inhomogeneous field mechanism in ferroelectric polymers," Journal of Physics D: Applied Physics 45, 165301 (2012).

${ }^{135}$ F. Preisach, "Über die magnetische Nachwirkung," Zeitschrift für Physik 94, 277-302 (1935).

${ }^{136}$ I. D. Mayergoyz, Mathematical Models of Hysteresis (Springer New York, New York, NY, 1991).

${ }^{137}$ A. V. Turik, "Theory of polarization and hysteresis of ferroelectrics," Sov. Physics Solid State 5, 885-886 (1963).

${ }^{138}$ G. Robert, D. Damjanovic, N. Setter, and A. V. Turik, "Preisach modeling of piezoelectric nonlinearity in ferroelectric ceramics," Journal of Applied Physics 89, 5067-5074 (2001).

${ }^{139}$ A. A. T. Bartic, D. J. D. J. Wouters, H. E. Maes, J. T. Rickes, and R. M. R. M. Waser, "Preisach model for the simulation of ferroelectric capacitors," Journal of Applied Physics 89, 3420-3425 (2001). 
${ }^{140}$ L. Cima, E. Labouré, and P. Muralt, "Characterization and model of ferroelectrics based on experimental Preisach density," Review of Scientific Instruments 73, 3546 (2002).

${ }^{141}$ L. Cima and E. Labouré, "A model of ferroelectric behavior based on a complete switching density," Journal of Applied Physics 95, 2654-2659 (2004).

${ }^{142}$ L. Wang, J. Yu, Y. Wang, G. Peng, F. Liu, and J. Gao, "Modeling ferroelectric capacitors based on the dipole switching theory," Journal of Applied Physics 101, 104505 (2007).

${ }^{143}$ F. Yang, M. H. Tang, Y. C. Zhou, X. J. Zheng, F. Liu, J. X. Tang, J. J. Zhang, J. Zhang, and C. Q. Sun, "A model for the polarization hysteresis loops of the perovskite-type ferroelectric thin films," Applied Physics Letters 91, 3-5 (2007).

${ }^{144}$ C. H. Tsang, B. Ploss, and F. G. Shin, "Simulation of the poling of P(VDFTrFE) with ferroelectric electrodes based on the Preisach model," Ferroelectrics 259, 139-144 (2001).

${ }^{145} \mathrm{C}$. H. Tsang, "Application of the preisach model to ferroelectric composites," PhD thesis (Hong Kong Polytechnic University, 2003).

${ }^{146}$ V. Khikhlovskyi, A. V. Gorbunov, A. J. J. M. van Breemen, R. A. J. Janssen, G. H. Gelinck, and M. Kemerink, "Multi-bit organic ferroelectric memory," Organic Electronics 14, 3399-3405 (2013).

${ }^{147}$ D. Zhao, I. Katsouras, K. Asadi, W. A. Groen, P. W. M. Blom, and D. M. de Leeuw, "Retention of intermediate polarization states in ferroelectric materials enabling memories for multi-bit data storage," Applied Physics Letters 108, 232907 (2016).

${ }^{148}$ Y. Kim, A. Kumar, O. S. Ovchinnikov, S. Jesse, H. Han, D. Pantel, I. Vrejoiu, W. Lee, D. Hesse, M. Alexe, and S. V. Kalinin, "First-Order Reversal Curve Probing of Spatially Resolved Polarization Switching Dynamics in Ferroelectric Nanocapacitors," ACS Nano 6, 491-500 (2012).

${ }^{149}$ O. S. Ovchinnikov, S. Jesse, S. Guo, K. Seal, P. Bintachitt, I. Fujii, S. Trolier-Mckinstry, and S. V. Kalinin, "Local measurements of Preisach density in polycrystalline ferroelectric capacitors using piezoresponse force spectroscopy," Applied Physics Letters 96, 491-500 (2010).

${ }^{150}$ S. Guo, O. S. Ovchinnikov, M. E. Curtis, M. B. Johnson, S. Jesse, and S. V. Kalinin, "Spatially resolved probing of Preisach density in polycrystalline ferroelectric thin films," Journal of Applied Physics 108, 084103 (2010).

${ }^{151}$ J. Lee, A. J. J. M. van Breemen, V. Khikhlovskyi, M. Kemerink, R. A. J. Janssen, and G. H. Gelinck, "Pulse-modulated multilevel data storage in an organic ferroelectric resistive memory diode," Scientific Reports 6, 24407 (2016). 
${ }^{152}$ A. Bortz, M. Kalos, and J. Lebowitz, "A new algorithm for Monte Carlo simulation of Ising spin systems," Journal of Computational Physics 17, 10-18 (1975).

${ }^{153}$ H. M. Duiker and P. D. Beale, "Grain-size effects in ferroelectric switching," Physical Review B 41, 490-495 (1990).

${ }^{154}$ B. G. Potter, V. Tikare, and B. A. Tuttle, "Monte Carlo simulation of ferroelectric domain structure and applied field response in two dimensions," Journal of Applied Physics 87, 4415-4424 (2000).

${ }^{155}$ C. L. Wang, Y. Xin, X. S. Wang, and W. L. Zhong, "Size effects of ferroelectric particles described by the transverse Ising model," Physical Review B 62, 11423-11427 (2000).

${ }^{156}$ C. J. Fennell and J. D. Gezelter, "Is the Ewald summation still necessary? Pairwise alternatives to the accepted standard for long-range electrostatics," The Journal of Chemical Physics 124, 234104 (2006).

${ }^{157}$ Y.-Z. Wu, D.-L. Yao, and Z.-Y. Li, "Monte-Carlo simulation of the switching behavior in ferroelectrics with dipolar defects," Solid State Communications 122, 395-400 (2002).

${ }^{158}$ D. Bolten, U. Böttger, and R. Waser, "Influence of Defects on the Properties of a 2D Ferroelectric: A Monte-Carlo Simulation Study," Japanese Journal of Applied Physics 41, 7202-7210 (2002).

${ }^{159}$ G. Ayton and G. Patey, "Ferroelectric Order in Model Discotic Nematic Liquid Crystals," Physical Review Letters 76, 239-242 (1996).

${ }^{160}$ R. Berardi, S. Orlandi, and C. Zannoni, "Monte Carlo simulation of discotic Gay-Berne mesogens with axial dipole," Journal of the Chemical Society, Faraday Transactions 93, 1493-1496 (1997).

${ }^{161}$ T. K. Bose and J. Saha, "Monte Carlo Simulations of Spontaneous Ferroelectric Order in Discotic Liquid Crystals," Physical Review Letters 110, 265701 (2013).

${ }^{162}$ M. Kühn and H. Kliem, "Monte Carlo Simulations of Ferroelectric Properties for PVDF and BaTiO 3," Ferroelectrics 370, 207-218 (2008).

${ }^{163}$ H. Kliem and M. Kühn, "Modeling the switching kinetics in ferroelectrics," Journal of Applied Physics 110, 114106 (2011).

${ }^{164}$ A. Leschhorn, S. Djoumbou, and H. Kliem, "Microscopic model of domain wall motion," Journal of Applied Physics 115, 114106 (2014).

${ }^{165}$ A. Leschhorn and H. Kliem, "Influence of thermal vibrations on polarization switching in the model of local fields," Journal of Applied Physics 121, 014103 (2017). 
${ }^{166}$ A. Simonov, T. Weber, and W. Steurer, "Experimental uncertainties of three-dimensional pair distribution function investigations exemplified on the diffuse scattering from a tris- tert -butyl-1,3,5-benzene tricarboxamide single crystal," Journal of Applied Crystallography 47, 2011-2018 (2014).

${ }^{167}$ C. S. Zehe, J. A. Hill, N. P. Funnell, K. Kreger, K. P. Van der Zwan, A. L. Goodwin, H. Schmidt, and J. Senker, "Mesoscale Polarization by Geometric Frustration in Columnar Supramolecular Crystals," Angewandte Chemie International Edition 56, 4432-4437 (2017).

${ }^{168}$ H. von Seggern and S. N. Fedosov, "Conductivity-induced polarization buildup in poly(vinylidene fluoride)," Applied Physics Letters 81, 28302832 (2002).

${ }^{169}$ M. Sepliarsky, A. Asthagiri, S. Phillpot, M. Stachiotti, and R. Migoni, "Atomic-level simulation of ferroelectricity in oxide materials," Current Opinion in Solid State and Materials Science 9, 107-113 (2005).

${ }^{170}$ S. R. Phillpot, S. B. Sinnott, and A. Asthagiri, "Atomic-Level Simulation of Ferroelectricity in Oxides: Current Status and Opportunities," Annual Review of Materials Research 37, 239-270 (2007).

${ }^{171}$ R. E. Cohen, "Theory of Ferroelectrics: A vision for the next decade and beyond," in Ferroelectricity, Vol. 61 (Wiley-VCH Verlag GmbH, Weinheim, Germany, 2007), pp. 139-154.

${ }^{172}$ U. V. Waghmare, "First-Principles Theory, Coarse-Grained Models, and Simulations of Ferroelectrics," Accounts of Chemical Research 47, 32423249 (2014).

${ }^{173}$ S. Tinte, M. G. Stachiotti, M. Sepliarsky, R. L. Migoni, and C. O. Rodriguez, "Atomistic modelling of BaTiO 3 based on first-principles calculations," Journal of Physics: Condensed Matter 11, 9679-9690 (1999).

${ }^{174}$ M. Sepliarsky, S. R. Phillpot, S. K. Streiffer, M. G. Stachiotti, and R. L. Migoni, "Polarization reversal in a perovskite ferroelectric by moleculardynamics simulation," Applied Physics Letters 79, 4417-4419 (2001).

${ }^{175}$ J. Paul, T. Nishimatsu, Y. Kawazoe, and U. V. Waghmare, "Ferroelectric Phase Transitions in Ultrathin Films of BaTiO3," Physical Review Letters 99, 077601 (2007).

${ }^{176}$ T. Nishimatsu, U. V. Waghmare, Y. Kawazoe, and D. Vanderbilt, "Fast molecular-dynamics simulation for ferroelectric thin-film capacitors using a first-principles effective Hamiltonian," Physical Review B 78, 104104 (2008).

${ }^{177}$ Publication list of feram, http://loto. sourceforge.net/feram/.

${ }^{178}$ Y.-H. Shin, V. R. Cooper, I. Grinberg, and A. M. Rappe, "Development of a bond-valence molecular-dynamics model for complex oxides," Physical Review B 71, 054104 (2005). 
${ }^{179}$ D. B. Korlepara and S. Balasubramanian, "Molecular modelling of supramolecular one dimensional polymers," RSC Advances 8, 22659-22669 (2018).

${ }^{180}$ K. K. Bejagam and S. Balasubramanian, "Supramolecular Polymerization: A Coarse Grained Molecular Dynamics Study," The Journal of Physical Chemistry B 119, 5738-5746 (2015).

${ }^{181}$ K. K. Bejagam, C. Kulkarni, S. J. George, and S. Balasubramanian, "External electric field reverses helical handedness of a supramolecular columnar stack," Chemical Communications 51, 16049-16052 (2015).

${ }^{182}$ P. J. Hasnip, K. Refson, M. I. J. Probert, J. R. Yates, S. J. Clark, and C. J. Pickard, "Density functional theory in the solid state," Philosophical Transactions of the Royal Society A: Mathematical, Physical and Engineering Sciences 372, 20130270 (2014).

${ }^{183}$ R. Resta, "Ab initio simulation of the properties of ferroelectric materials," Modelling and Simulation in Materials Science and Engineering 11, R69R96 (2003).

${ }^{184}$ K. M. Rabe, C. H. Ahn, and J.-M. Triscone, Physics of Ferroelectrics, Vol. 105, Topics in Applied Physics (Springer Berlin Heidelberg, Berlin, Heidelberg, 2007).

${ }^{185}$ R. E. Cohen and H. Krakauer, "Lattice dynamics and origin of ferroelectricity in BaTiO3: Linearized-augmented-plane-wave total-energy calculations," Physical Review B 42, 6416-6423 (1990).

${ }^{186}$ R. Resta, M. Posternak, and A. Baldereschi, "Towards a quantum theory of polarization in ferroelectrics: The case of KNbO3," Physical Review Letters 70, 1010-1013 (1993).

${ }^{187}$ G. Sághi-Szabó, R. E. Cohen, and H. Krakauer, "First-Principles Study of Piezoelectricity in PbTiO3," Physical Review Letters 80, 4321-4324 (1998).

${ }^{188}$ J. Junquera and P. Ghosez, "Critical thickness for ferroelectricity in perovskite thin films," Nature 422, 506-509 (2003).

${ }^{189}$ M. Stengel, D. Vanderbilt, and N. A. Spaldin, "First-principles modeling of ferroelectric capacitors via constrained displacement field calculations," Physical Review B 80, 224110 (2009).

${ }^{190}$ P. Aguado-Puente and J. Junquera, "Ferromagneticlike Closure Domains in Ferroelectric Ultrathin Films: First-Principles Simulations," Physical Review Letters 100, 177601 (2008).

${ }^{191}$ T. Shimada, S. Tomoda, and T. Kitamura, "Ab initio study of ferroelectric closure domains in ultrathin PbTiO 3 films," Physical Review B 81, 144116 (2010). 
${ }^{192}$ H.-Y. Ye, Y.-Y. Tang, P.-F. Li, W.-Q. Liao, J.-X. Gao, X.-N. Hua, H. Cai, P.-P. Shi, Y.-M. You, and R.-G. Xiong, "Metal-free three-dimensional perovskite ferroelectrics.," Science 361, 151-155 (2018).

${ }^{193}$ S. M. Nakhmanson, M. B. Nardelli, and J. Bernholc, "Collective polarization effects in $\beta$-polyvinylidene fluoride and its copolymers with tri- and tetrafluoroethylene," Physical Review B 72, 115210 (2005).

${ }^{194}$ I. A. W. Filot, A. R. A. Palmans, P. a.J. Hilbers, R. A. Van Santen, E. A. Pidko, and T. F. A. de Greef, "Understanding cooperativity in hydrogenbond-induced supramolecular polymerization: A density functional theory study," Journal of Physical Chemistry B 114, 13667-13674 (2010).

${ }^{195}$ C. Kulkarni, S. K. Reddy, S. J. George, and S. Balasubramanian, "Cooperativity in the stacking of benzene-1,3,5-tricarboxamide: The role of dispersion," Chemical Physics Letters 515, 226-230 (2011).

${ }^{196}$ J. F. Scott, Ferroelectric Memories, Vol. 3, Springer Series in Advanced Microelectronics (Springer Berlin Heidelberg, Berlin, Heidelberg, 2000).

${ }^{197}$ J. F. Scott, “Applications of Modern Ferroelectrics," Science 315, 954-959 (2007).

${ }^{198}$ K. Asadi, "Resistance switching in two-terminal ferroelectricsemiconductor lateral heterostructures," Applied Physics Reviews 7, 021307 (2020).

${ }^{199}$ B. Park, Ferroelectric-Gate Field Effect Transistor Memories, edited by B.-E. Park, H. Ishiwara, M. Okuyama, S. Sakai, and S.-M. Yoon, Vol. 131, Topics in Applied Physics (Springer Singapore, Singapore, 2020).

${ }^{200}$ J. C. Scott, "MATERIALS SCIENCE: Is There an Immortal Memory?" Science 304, 62-63 (2004).

${ }^{201}$ V. Khikhlovskyi, A. J. J. M. van Breemen, R. A. J. Janssen, G. H. Gelinck, and M. Kemerink, "Data retention in organic ferroelectric resistive switches," Organic Electronics 31, 56-62 (2016).

${ }^{202}$ A. J. J. M. van Breemen, J.-L. van der Steen, G. van Heck, R. Wang, V. Khikhlovskyi, M. Kemerink, and G. H. Gelinck, "Crossbar arrays of nonvolatile, rewritable polymer ferroelectric diode memories on plastic substrates," Applied Physics Express 7, 031602 (2014).

${ }^{203}$ M. Kemerink, K. Asadi, P. W. M. Blom, and D. M. de Leeuw, "The operational mechanism of ferroelectric-driven organic resistive switches," Organic Electronics 13, 147-152 (2012).

${ }^{204}$ M. Ghittorelli, T. Lenz, H. Sharifi Dehsari, D. Zhao, K. Asadi, P. W. M. Blom, Z. M. Kovács-Vajna, D. M. de Leeuw, and F. Torricelli, "Quantum tunnelling and charge accumulation in organic ferroelectric memory diodes," Nature Communications 8, 15741 (2017). 
${ }^{205}$ A. van Breemen, T. Zaba, V. Khikhlovskyi, J. Michels, R. Janssen, M. Kemerink, and G. Gelinck, "Surface Directed Phase Separation of Semiconductor Ferroelectric Polymer Blends and their Use in Non-Volatile Memories," Advanced Functional Materials 25, 278-286 (2015).

${ }^{206}$ P. W. M. Blom, R. M. Wolf, J. F. M. Cillessen, and M. P. C. M. Krijn, "Ferroelectric Schottky Diode," Physical Review Letters 73, 2107-2110 (1994).

${ }^{207}$ V. Garcia and M. Bibes, "Ferroelectric tunnel junctions for information storage and processing," Nature Communications 5, 1-12 (2014).

${ }^{208}$ R. Guo, W. Lin, X. Yan, T. Venkatesan, and J. Chen, "Ferroic tunnel junctions and their application in neuromorphic networks," Applied Physics Reviews 7, 011304 (2020).

${ }^{209}$ Y. Yuan, Z. Xiao, B. Yang, and J. Huang, "Arising applications of ferroelectric materials in photovoltaic devices," Journal of Materials Chemistry A 2, 6027 (2014).

${ }^{210}$ K. T. Butler, J. M. Frost, and A. Walsh, "Ferroelectric materials for solar energy conversion: photoferroics revisited," Energy Environ. Sci. 8, 838848 (2015).

${ }^{211}$ C. Paillard, X. Bai, I. C. Infante, M. Guennou, G. Geneste, M. Alexe, J. Kreisel, and B. Dkhil, "Photovoltaics with Ferroelectrics: Current Status and Beyond," Advanced Materials 28, 5153-5168 (2016).

${ }^{212}$ P. Lopez-Varo, L. Bertoluzzi, J. Bisquert, M. Alexe, M. Coll, J. Huang, J. A. Jimenez-Tejada, T. Kirchartz, R. Nechache, F. Rosei, and Y. Yuan, "Physical aspects of ferroelectric semiconductors for photovoltaic solar energy conversion," Physics Reports 653, 1-40 (2016).

${ }^{213}$ T. Choi, S. Lee, Y. J. Choi, V. Kiryukhin, and S.-W. Cheong, "Switchable Ferroelectric Diode and Photovoltaic Effect in BiFeO3," Science 324, 6366 (2009).

${ }^{214}$ R. Guo, L. You, Y. Zhou, Z. S. Lim, X. Zou, L. Chen, R. Ramesh, and J. Wang, "Non-volatile memory based on the ferroelectric photovoltaic effect.," Nature communications 4, 1990 (2013).

${ }^{215}$ R. Nechache, C. Harnagea, S. Li, L. Cardenas, W. Huang, J. Chakrabartty, and F. Rosei, "Bandgap tuning of multiferroic oxide solar cells," Nature Photonics 9, 61-67 (2015).

${ }^{216}$ J. Guilleme, J. Aragó, E. Ortí, E. Cavero, T. Sierra, J. Ortega, C. L. Folcia, J. Etxebarria, D. González-Rodríguez, and T. Torres, "A columnar liquid crystal with permanent polar order," Journal of Physical Chemistry C 3, 985-989 (2015). 
${ }^{217}$ C. Zhang, K. Nakano, M. Nakamura, F. Araoka, K. Tajima, and D. Miyajima, "Noncentrosymmetric Columnar Liquid Crystals with the Bulk Photovoltaic Effect for Organic Photodetectors," Journal of the American Chemical Society, jacs.9b12710 (2020).

${ }^{218}$ Y. Funatsu, A. Sonoda, and M. Funahashi, "Ferroelectric liquid-crystalline semiconductors based on a phenylterthiophene skeleton: effect of the introduction of oligosiloxane moieties and photovoltaic effect," Journal of Materials Chemistry C 3, 1982-1993 (2015).

${ }^{219}$ A. Seki and M. Funahashi, "Photovoltaic Effects in Ferroelectric Liquid Crystals based on Phenylterthiophene Derivatives," Chemistry Letters 45, 616-618 (2016).

${ }^{220}$ A. Seki, Y. Funatsu, and M. Funahashi, "Anomalous photovoltaic effect based on molecular chirality: influence of enantiomeric purity on the photocurrent response in $\pi$-conjugated ferroelectric liquid crystals," Physical Chemistry Chemical Physics 19, 16446-16455 (2017).

${ }^{221}$ J. Wu, T. Takeda, N. Hoshino, Y. Suzuki, J. Kawamata, and T. Akutagawa, "Ferroelectricity of a Tetraphenylporphyrin Derivative Bearing -CONHC 14 H 29 Chains at $500 \mathrm{~K}$," The Journal of Physical Chemistry C 123, 22439-22446 (2019).

${ }^{222}$ H. Anetai, T. Takeda, N. Hoshino, Y. Araki, T. Wada, S. Yamamoto, M. Mitsuishi, H. Tsuchida, T. Ogoshi, and T. Akutagawa, "Circular Polarized Luminescence of Hydrogen-Bonded Molecular Assemblies of Chiral Pyrene Derivatives," The Journal of Physical Chemistry C 122, 6323-6331 (2018).

${ }^{223}$ H. Anetai, Y. Wada, T. Takeda, N. Hoshino, S. Yamamoto, M. Mitsuishi, T. Takenobu, and T. Akutagawa, "Fluorescent Ferroelectrics of HydrogenBonded Pyrene Derivatives," The Journal of Physical Chemistry Letters 6, 1813-1818 (2015).

${ }^{224}$ T. Takeda, J. Wu, N. Ikenaka, N. Hoshino, I. Hisaki, and T. Akutagawa, "C 3 Symmetric Hexaphenyltriphenylenehexamide: Molecular Design of Fluorescent Ferroelectrics," ChemistrySelect 3, 10608-10614 (2018).

${ }^{225}$ A. Köhler and H. Bässler, Electronic Processes in Organic Semiconductors (Wiley-VCH Verlag GmbH \& Co. KGaA, Weinheim, Germany, 2015).

${ }^{226}$ A. Miller and E. Abrahams, "Impurity Conduction at Low Concentrations," Physical Review 120, 745-755 (1960).

${ }^{227}$ R. A. Marcus, "On the Theory of Oxidation-Reduction Reactions Involving Electron Transfer. I," The Journal of Chemical Physics 24, 966-978 (1956).

${ }^{228}$ H. Bässler, "Charge Transport in Disordered Organic Photoconductors a Monte Carlo Simulation Study," physica status solidi (b) 175, 15-56 (1993). 


\section{Papers and Contributions}

\section{Papers Included in This Thesis}

${ }^{1}$ A. V. Gorbunov, M. García-Iglesias, J. Guilleme, T. D. Cornelissen, W. S. C. Roelofs, T. Torres, D. González-Rodríguez, E. W. Meijer, and M. Kemerink, "Ferroelectric self-assembled molecular materials showing both rectifying and switchable conductivity," Science Advances 3, e1701017 (2017).

${ }^{2}$ I. Urbanavičiūtè, T. D. Cornelissen, X. Meng, R. P. Sijbesma, and M. Kemerink, "Physical reality of the Preisach model for organic ferroelectrics," Nature Communications 9, 4409 (2018).

${ }^{3}$ T. D. Cornelissen, M. Biler, I. Urbanaviciute, P. Norman, M. Linares, and M. Kemerink, "Kinetic Monte Carlo simulations of organic ferroelectrics," Physical Chemistry Chemical Physics 21, 1375-1383 (2019).

${ }^{4}$ T. D. Cornelissen, I. Urbanaviciute, and M. Kemerink, "Microscopic model for switching kinetics in organic ferroelectrics following the Merz law," Physical Review B 101, 214301 (2020).

\section{Author's Contributions to the Included Papers}

1. Together with M.K. developed the model for asymmetric Marcus hopping. Performed simulations with this model, analyzed the results, and compared them to the experiments. Wrote the modeling part of the manuscript. Revised the manuscript draft together with the coauthors.

2. Performed the Preisach distribution measurements and analysis. Developed and performed simulations with the kinetic Monte Carlo model. 
Together with the coauthors developed the analytical model used for analyzing the results. Wrote the manuscript together with I.U. and revised the drafts with all coauthors.

3. Developed the kinetic Monte Carlo model and wrote the underlying code. Performed the kMC simulations and analyzed the results. Wrote the manuscript draft, and revised it together with the coauthors.

4. Expanded on the kinetic Monte Carlo model and wrote the underlying code. Performed the kMC simulations and analyzed the results. Developed and applied the analytical model. Wrote the manuscript draft, and revised it together with the coauthors.

\section{Papers Not Included in This Thesis}

${ }^{5}$ I. Urbanavičiūtè, X. Meng, T. D. Cornelissen, A. V. Gorbunov, S. Bhattacharjee, R. P. Sijbesma, and M. Kemerink, "Tuning the Ferroelectric Properties of Trialkylbenzene-1,3,5-tricarboxamide (BTA)," Advanced Electronic Materials, 1600530 (2017).

${ }^{6}$ I. Urbanavičiūtè, S. Bhattacharjee, M. Biler, J. A. M. Lugger, T. D. Cornelissen, P. Norman, M. Linares, R. P. Sijbesma, M. Kemerink, I. Urbanaviciute, S. Bhattacharjee, M. Biler, J. A. M. Lugger, T. D. Cornelissen, P. Norman, M. Linares, R. P. Sijbesma, and M. Kemerink, "Suppressing depolarization by tail substitution in an organic supramolecular ferroelectric," Physical Chemistry Chemical Physics 21, 2069-2079 (2019).

${ }^{7}$ I. Urbanavičiūtè, X. Meng, M. Biler, Y. Wei, T. D. Cornelissen, S. Bhattacharjee, M. Linares, and M. Kemerink, "Negative piezoelectric effect in an organic supramolecular ferroelectric," Materials Horizons 6, 1688-1698 (2019).

${ }^{8}$ N. M. Casellas, I. Urbanaviciute, T. D. Cornelissen, J. A. Berrocal, T. Torres, M. Kemerink, and M. García-Iglesias, "Resistive switching in an organic supramolecular semiconducting ferroelectric," Chemical Communications 55, 8828-8831 (2019). 


\section{Papers}

The papers associated with this thesis have been removed for copyright reasons. For more details about these see:

http://urn.kb.se/resolve?urn=urn:nbn:se:liu:diva-167271 


\section{FACULTY OF SCIENCE AND ENGINEERING}

Linköping Studies in Science and Technology, Dissertation No. 2080, 2020 Department of Physics, Chemistry, and Biology

Linköping University

SE-581 83 Linköping, Sweden

www.liu.se 